\title{
Bilateral processing of thermoreception in the olfactory system of larval Xenopus laevis
}

Dissertation zur Erlangung des mathematisch-naturwissenschaftlichen Doktorgrades

"Doctor rerum naturalium"

der Georg-August-Universität Göttingen

vorgelegt von

Eugen Kludt

aus Alma-Ata, Kasachstan

Göttingen 2009 
Mitglied des Betreuungsausschusses (Referent): Prof. Dr. Dr. Detlev Schild, Universität Göttingen, Abteilung Neurophysiologie und Zelluläre Biophysik

Mitglied des Betreuungsausschusses (Referent): Prof. Dr. Ralf Heinrich, Universität Göttingen, Abteilung Neurobiologie

Mitglied des Betreuungsausschusses: Prof. Dr. Michael Müller, Universität Göttingen, Abteilung Neuro- und Sinnesphysiologie

Tag der mündlichen Prüfung: 
Herewith I declare, that I prepared the thesis "Bilateral processing of thermoreception in the olfactory system of larval Xenopus laevis" on my own and with no other sources and aids than quoted.

Göttingen, November $26^{\text {th }} 2009$ 

Mapure 



\section{Table of Contents}

1. Introduction. 1

1.1 What is an adequate quality for the olfactory system?..................................

1.2 General overview of the vertebrate olfactory systems........................................

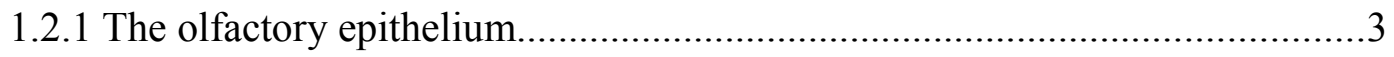

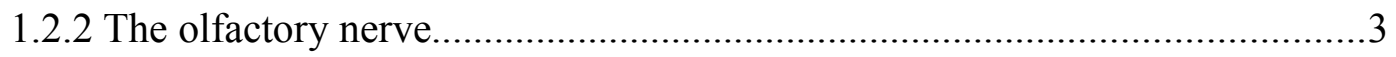

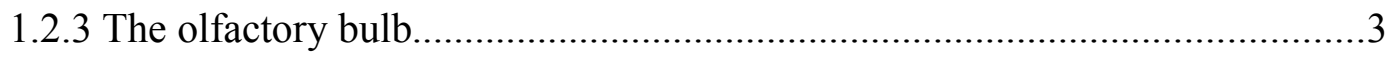

1.3 Olfactory system of larval Xenopus laevis.......................................................

1.3.1 Development of the olfactory epithelium.....................................................4

1.3.2 Development and functional organisation of the olfactory bulb...................5

1.3.3 Morphology of the primary olfactory projection in larval Xenopus laevis.....8

1.4 Bilateral processing in the olfactory system................................................... 10

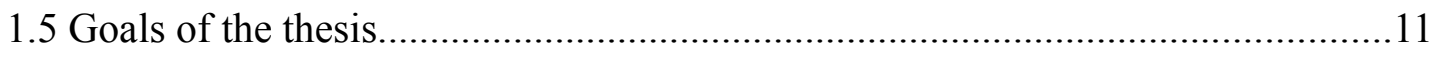

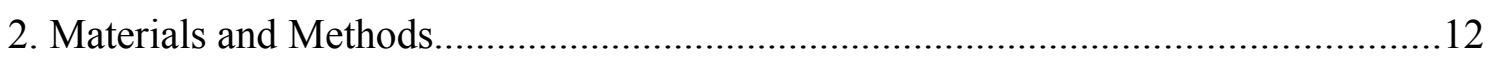

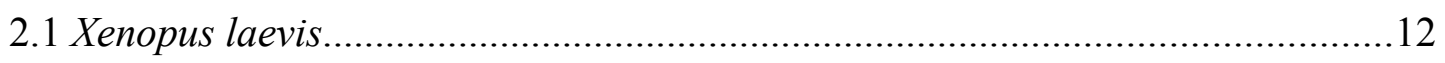

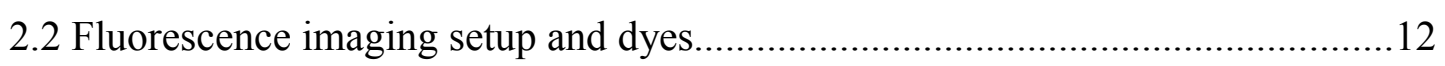

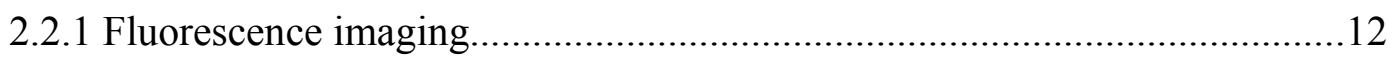

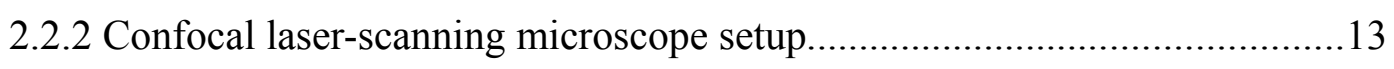

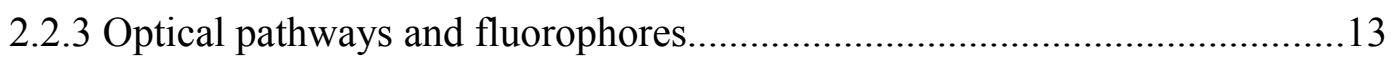

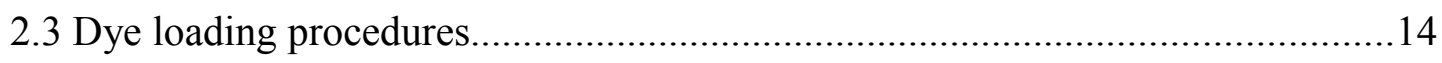

2.3.1 Electroporation loading procedure......................................................... 14

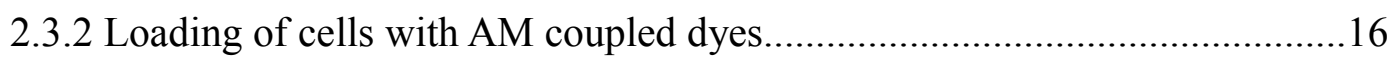

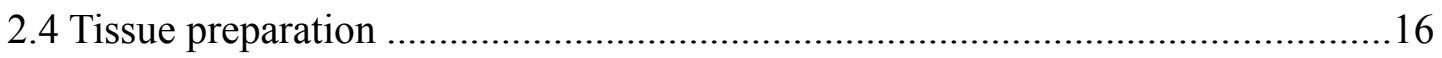

2.4.1 Sliced preparation and bath incubation staining...................................... 16

2.4.2 Explanted nose-olfactory bulb preparation and bolus loading.....................17

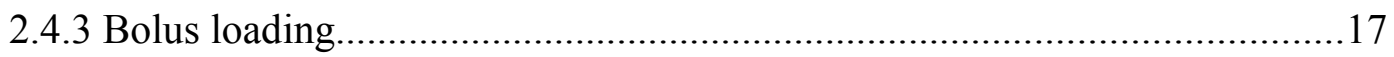

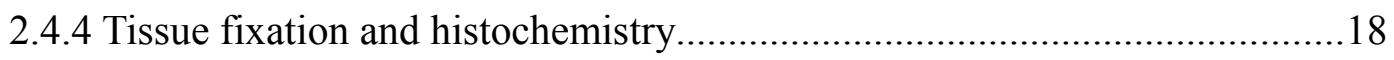

2.5 Stimulation of the olfactory epithelium........................................................ 18

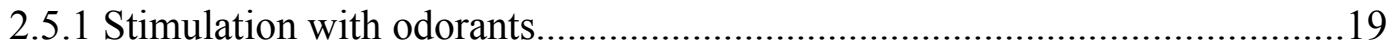

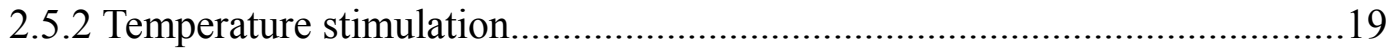

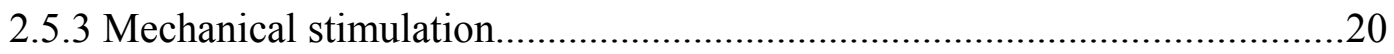

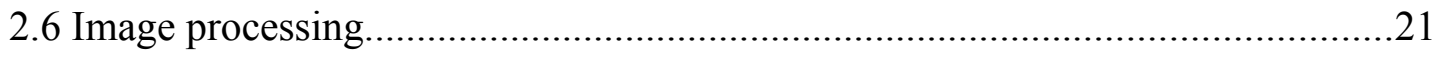

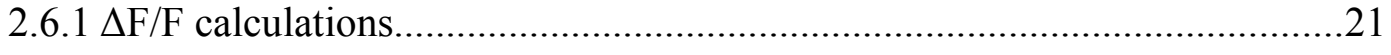

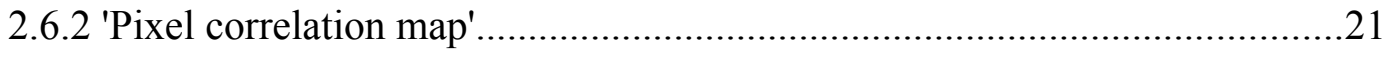

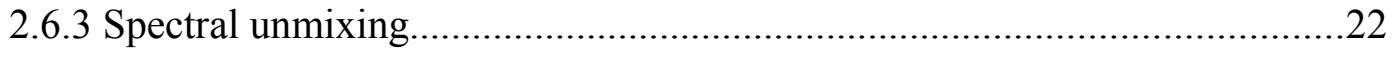




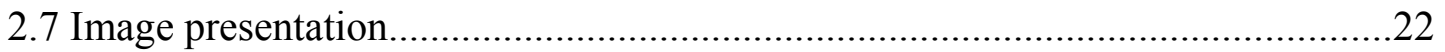

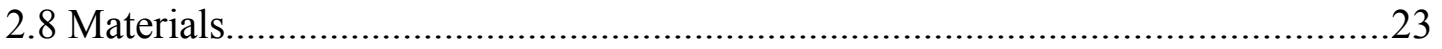

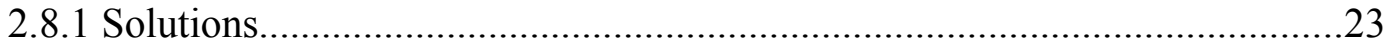

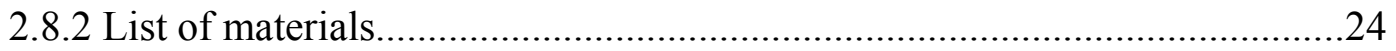

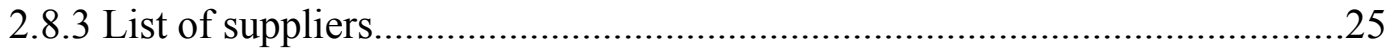

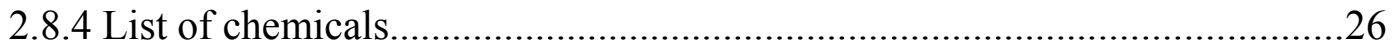

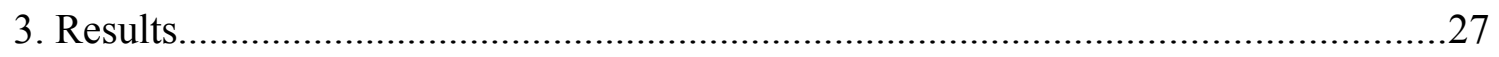

3.1 Morphology of the contralateral projecting axons of olfactory sensory neurons. 27

3.1.1 The olfactory bulb is innervated by the contralateral olfactory epithelium

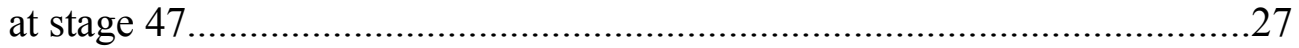

3.1.2 Two different contralateral innervation pathways for the axons of

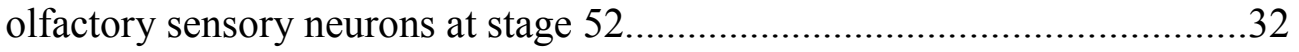

3.1.3 Mapping of deviating axons of olfactory sensory neurons at stage $52 \ldots \ldots \ldots . .35$

3.1.4 Characterisation of axons of olfactory sensory neurons invading the contralateral olfactory bulb across the fusion zone of PF9.........................38

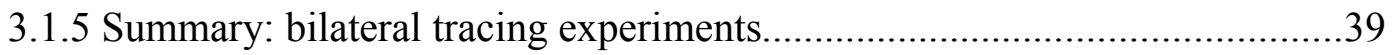

3.2 Calcium imaging of axons of olfactory sensory neurons within

the olfactory bulb.

3.2.1 $\left[\mathrm{Ca}^{2+}\right]_{\mathrm{i}}$ transients are not restricted to the presynaptic terminals of

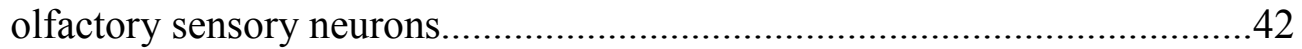

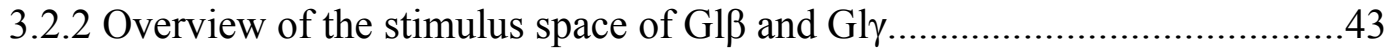

3.2.3 Spatial distribution of the G1 $\beta$ response to stimulation with amino acids

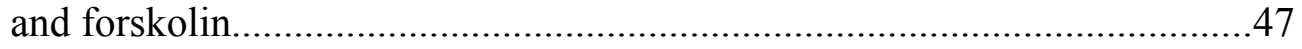

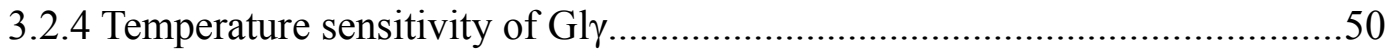

3.2.5 Summary: calcium imaging experiments with axons of olfactory sensory neurons..

3.3 Calcium imaging of processes and somata of mitral cells..

3.3.1 Determination of the calcium sensitive dye distribution within the olfactory bulb after bulk staining....

3.3.2 Response profiles of mitral cell somata and their processes upon temperature stimulation

3.3.3 Responses to contralateral temperature stimulation within the olfactory bulb...

3.3.4 Summary: results of calcium imaging of mitral cells...............................72

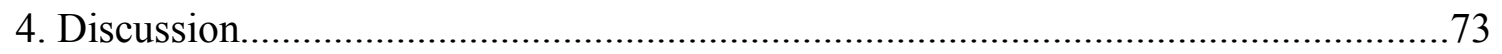

4.1 Are axons of olfactory sensory neurons the exclusive constituents of

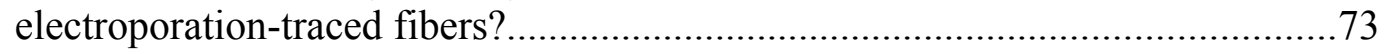

4.2 Deviating axons of olfactory sensory neurons - a new olfactory pathway?.........75 
4.3 Is Gl $\gamma$ an exclusive target for deviating axons of olfactory sensory neurons?......76

4.4 Is temperature an adequate quality for the vertebrate olfactory system?..............77

4.5 What is the function of the contralateral innervation?.......................................78

4.6 Calcium influx over the entire length of axons of olfactory sensory neurons......79

4.7 Selective postsynaptic staining by AM dyes....................................................80

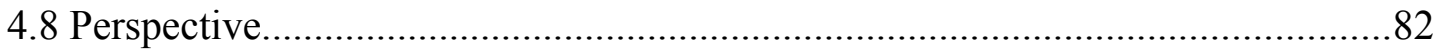

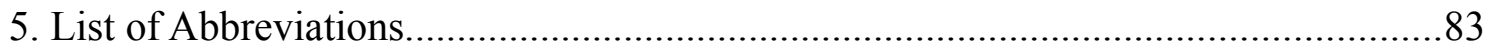

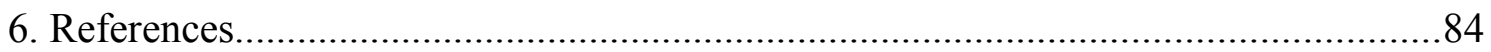

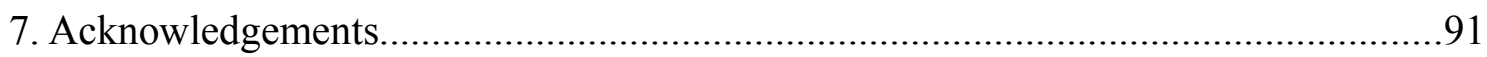

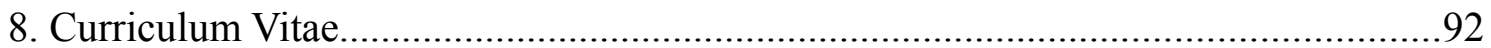




\section{List of Figures}

Figure 1.1: Schematic parasagittal sections through the olfactory system of larval Xenopus laevis. .5

Figure 1.2: Schematic overview of the main olfactory bulb of Xenopus laevis................ 7

Figure 1.3: Two nomenclatures for primary olfactory projections.................................9

Figure 3.1: Overview of bilateral projections of axons of olfactory sensory neurons at stage 47 ......

Figure 3.2: Glomerular tuft structure formed by axons of olfactory sensory neurons

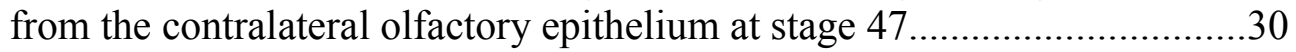

Figure 3.3: Overview of projections of olfactory sensory neurons within the olfactory bulb at stage 52

Figure 3.4: Projection of deviating axons of olfactory sensory neurons via the anterior commissure at stage 52 .

Figure 3.5: Specific innervation of Gl $\gamma$ by deviating axons of olfactory sensory neurons at stage 52 .

Figure 3.6: The direct contralateral innervation within the dorsomedial cluster.

Figure 3.7: $\left[\mathrm{Ca}^{2+}\right]_{\mathrm{i}}$ transients within the olfactory nerve as a response to odorant stimulation.

Figure 3.8: Overview of stimulus specificity in Gl $\beta$ and Gl $\gamma$.

Figure 3.9: Response of G1 $\beta$ to mechanical stimulation.

Figure 3.10: Response of G1 $\beta$ to stimulation with amino acids and forskolin. .48

Figure 3.11: Responses of ipsilateral presynaptic terminals of Gl $\gamma$ to temperature stimulation

Figure 3.12: Temperature sensitivity of deviating axons of olfactory sensory neurons. .55

Figure 3.13: Fluorescence distribution after bolus loading with Fura Red/AM..............56

Figure 3.14: Fluorescence distribution after bath incubation with Fura Red/AM...........58

Figure 3.15: Fluorescence distribution after bolus loading with fluo-4/AM .................61

Figure 3.16: Fluorescence distribution after bath incubation with fluo-4/AM ..............62

Figure 3.17: Temperature sensitivity of postsynaptic terminals of Gl $\gamma$

Figure 3.18: $\Delta \mathrm{F} / \mathrm{F}$ peak response maps during stimulation with different temperatures within the bulk stained olfactory bulb.

Figure 3.19: Response traces to graduated temperature stimulation within the bulk stained olfactory bulb.

Figure 3.20: Responses to contralateral temperature stimulation within the olfactory bulb. 


\section{List of Tables}

Table 1: Innervation of different glomerular structures by deviating axons of olfactory sensory neurons at stage 52 .

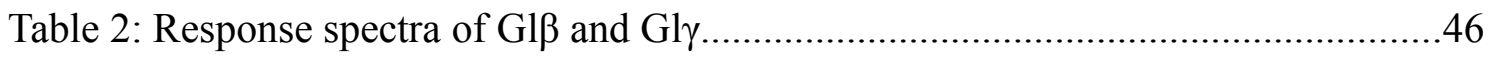





\section{Abstract}

This study of the ventral olfactory bulb of larval Xenopus laevis shows for the first time the existence of a thermosensitive olfactory glomerulus. This thermosensitive olfactory glomerulus was found to be innervated by axons of olfactory sensory neurons emerging from the ipsilateral olfactory epithelium as well as from the contralateral olfactory epithelium. The perfusion of the ipsilateral or contralateral olfactory epithelium with cold, odor-free Ringer solution elicited a transient $\left[\mathrm{Ca}^{2+}\right]_{\mathrm{i}}$ response within the axons of olfactory sensory neurons and in mitral cells. The activation of mitral cells demonstrated that the response to temperature stimulation is transmitted across the first olfactory synapse. The thermoreception was spatially restricted to this glomerulus, which did not respond to any of the tested odors. Adjacent olfactory glomeruli responded to olfactory and mechanical stimulation without showing any response to temperature changes. The spatially restricted, bilateral processing of temperature stimuli at the level of the olfactory glomerulus and mitral cells indicates that temperature represents a novel, adequate quality for the vertebrate olfactory system. 



\section{Introduction}

\subsection{What is an adequate quality for the olfactory system?}

The chemosensory neurons of the vertebrate olfactory system are able to recognize and discriminate thousands of molecules (Eisthen, 2002). During vertebrate evolution the olfactory system developed into different subsystems to suit specific needs. The first ontogenetic division into two subsystems, the main and accessory olfactory systems, emerged in early tetrapods before they conquered terrestric habitats (Eisthen, 1997). The accessory olfactory system is generally, but not exclusively (Baxi et al., 2006), associated with pheromone perception (Eisthen \& Wyatt, 2006). Further subdivision of the main olfactory system was caused by different conditions for sensing volatile molecules in a terrestrial environment. Semiaquatic vertebrates like amphibians employ different subsystems for these two environmental conditions as shown for adult Xenopus laevis (Freitag et al., 1995; Freitag et al., 1998; Weiss, 1986).

Xenopus laevis tadpoles are purely aquatic animals until their metamorphosis is finalised. The olfactory sensory neurons (OSNs) of Xenopus laevis can be activated by several classes of water-soluble molecules. First, amino acids are known to be behaviourally relevant odorants (Kiseleva, 1995) and are commonly used for studies of the olfactory system in aquatic species (Caprio and Byrd, 1984; Kang and Caprio, 1995; Rolen et al., 2003; Sato and Suzuki, 2001) including zebrafish (Friedrich and Korsching, 1997; Friedrich and Korsching, 1998; Tabor et al., 2004; Yaksi et al., 2007) and Xenopus laevis (Czesnik et al., 2007; Manzini and Schild, 2003b; Manzini et al., 2007a; Manzini et al., 2002). Second, nucleotides were reported to cause calcium responses in both OSNs and sustentacular cells (Czesnik et al., 2006) of larval Xenopus laevis and were also successfully used as odorants for other aquatic species (Friedrich and Korsching, 1998; Kang and Caprio, 1995; Rolen et al., 2003). Third, amines, bile salts, gonadal steroids and prostaglandins are also reported as potent aquatic odorants (Friedrich and Korsching, 1998; Gliem et al., 2009; Kang and Caprio, 1995; Rolen and Caprio, 2008; Rolen et al., 2003; Sato and Suzuki, 2001).

The differentiation of the olfactory system into distinct subsystems follows the physical properties of the molecules sensed (water soluble or volatile) and their meaning for the animal (e.g. species-specific pheromones or general food molecules). But this generalized classification is not absolute. Amines are an example for an odorant that is 
volatile as well as water soluble (Gliem et al., 2009; Mori et al., 2006; Rolen and Caprio, 2007; Rolen et al., 2003). The main olfactory system was shown to mediate pheromone responses while the accessory olfactory system was shown to respond to food odors (Baxi et al., 2006).

There are several other intriguing subsystems with enigmatic functions in anamniotic vertebrates (e.g. extrabulbar olfactory fibers (EBOF) (Hofmann and Meyer, 1995; Pinelli et al., 2004) and the terminal nerve (Mousley et al., 2006)) as well as in mammals (e.g. the septal organ of Masera (Breer and Strotmann, 2005; Marshall and Maruniak, 1986) and the Grüneberg ganglion (Fuss et al., 2005; Grüneberg, 1973; Koos and Fraser, 2005; Roppolo et al., 2006; Storan and Key, 2006)). Recent reports even question that the chemical properties of a molecule are the exclusive adequate quality for the olfactory system. As an example, the olfactory system of goldfish is known to respond to mechanical stimulation (Schild, 1985). Additionally, a dual function of some OSNs as odor detectors and mechanical sensors is reported for the septal organ and the main olfactory epithelium in mouse (Grosmaitre et al., 2007). The function of this mechanosensitivity might be the integration of the odorant concentration and the airflow strength over the olfactory epithelium (OE) at the single-cell level. Mechanical stimulation increases the responses of individual OSNs to stimulation with weak odorants. Furthermore, the mechanosensitivity is involved in the synchronization between respiratory cycles and theta oscillations within the olfactory bulb (OB) (Grosmaitre et al., 2007).

Mechanical excitation is not the only additional quality that is discussed as an adequate stimulus for the olfactory system. The Grüneberg ganglion is a mammalian olfactory subsystem that lies at the dorsal tip of the nasal cavity, close to the opening of the naris (Fuss et al., 2005; Grüneberg, 1973; Koos and Fraser, 2005; Roppolo et al., 2006; Storan and Key, 2006). A recent study identified low temperature as a stimulus which triggers a response within this subsystem (Mamasuew et al., 2008). The studies by Grosmaitre et al. (2007) and Mamasuew et al. (2008) indicate that the detection of chemical stimuli may not be the only function of the olfactory system. 


\subsection{General overview of the vertebrate olfactory systems}

\subsubsection{The olfactory epithelium}

The olfactory epithelium of vertebrates is a pseudostratified epithelium inside the nasal cavity. It consists of three cell types: OSNs, sustentacular cells and basal cells.

Olfactory sensory neurons are bipolar neurons that extend their dendrites to the surface of the $\mathrm{OE}$ and innervate the OB with their axons. Most OSNs express canonical odorant receptors which can be differentiated into two phylogenetic classes (Spehr and Munger, 2009). Class I olfactory receptors are the most common olfactory receptors in fish and are thought to be activated by water-soluble odorants. Class II olfactory receptors are the most common olfactory receptors in terrestrial tetrapods and are generally thought to be activated by volatile odorants (Freitag et al., 1995; Freitag et al., 1998; Niimura and Nei, 2006).

Olfactory sensory neurons are embedded in sustentacular cells that share properties with both glial and epithelial cells (Hassenklöver et al., 2008). Basal cells provide the regenerative capacity for the OE (Graziadei, 1973).

\subsubsection{The olfactory nerve}

The olfactory nerve contains unmyelinated OSN axons in all vertebrates. These axons are packed into bundles, which are surrounded by processes of the ensheathing glial cells (Burd, 1991; Gasser, 1956). The speed of propagation of action potentials for poikilothermal vertebrates $(0.14 \mathrm{~m} / \mathrm{s}$ frog, Ottoson, 1960$)$ is two to three times slower than for homeotherms $(0.47 \mathrm{~m} / \mathrm{s}$ mouse, Griff et al., 2000) while the axon diameter is in the same range of about $0.2 \mu \mathrm{m}$ (Gasser, 1956; Phillips and Griff, 2002; Scott and Sherrill, 2008). This is in line with typical coefficients of temperature dependence $\left(Q_{10}\right)$ of two to three for this temperature difference in most biological systems (Phillips and Griff, 2002).

\subsubsection{The olfactory bulb}

The vertebrate $\mathrm{OB}$ consists of several layers with their boundaries being less distinct in the ancestral condition (Eisthen, 1997). The axons of OSNs innervate the OB and converge into olfactory glomeruli, where they branch and terminate on dendritic terminals of output neurons (mitral cells) and local interneurons (periglomerular cells). In anamniotic vertebrates, the mitral cells innervate several glomeruli with their 
dendrites (Eisthen, 1997). A second population of interneurons, granule cells, lies deeper within the OB and forms reciprocal synapses with mitral cells. Axons of mitral cells leave the OB by the medial olfactory pathway towards ipsilateral ventral forebrain areas (septum, striatum) or by the lateral olfactory pathway to bilaterally innervate lateral and dorsal pallial areas (Eisthen, 1997).

\subsection{Olfactory system of larval Xenopus laevis}

The adult Xenopus laevis has three functional olfactory subsystems: aquatic, aerial and accessory (Altner, 1962; Hansen et al., 1998; Meyer et al., 1996), which appear successively during tadpole development. The development of Xenopus laevis was divided into stages by Nieuwkoop and Faber (Nieuwkoop and Faber, 1994). Following their classification, the development of the olfactory system will be described in detail in the following.

\subsubsection{Development of the olfactory epithelium}

The olfactory epithelia of Xenopus laevis originate from olfactory placodes that start to formate at stage 23 (Nieuwkoop and Faber, 1994). First OSN axons reach the OB at stage 30 (Byrd and Burd, 1991) and the expression of the first, aquatic olfactory receptors is detectable from stage 32 (Mezler et al., 1999). The olfactory sensory neurons reach morphological maturity at approximately stage $37 / 38$ while the olfactory placodes start to segregate into the principal cavity and the vomeronasal organ at stage 40 (Nieuwkoop and Faber, 1994).

The main olfactory system develops into a fully functional aquatic olfactory system with stage 47 (Figure 1.1 A) (Gaudin and Gascuel, 2005). The reorganization towards an adult-like olfactory system begins with the interspersed expression of volatile olfactory receptors at stage 49 (Mezler et al., 1999). At stage 50 the choanae are formed (Nieuwkoop and Faber, 1994). The air nose becomes apparent at stages $51-52$ as the principal cavity segregates into the medial (MD) and lateral diverticulum (LD) (Weiss, 1986). At stage 54 the expression of receptors is eventually separated into the aquatic class I receptors within the LD and the volatile class II receptors within the MD (Mezler et al., 1999). The final spatial configuration of the LD and the MD is reached at the metamorphic climax after stage 58 (Figure 1.1 B) (Weiss, 1986). 
A

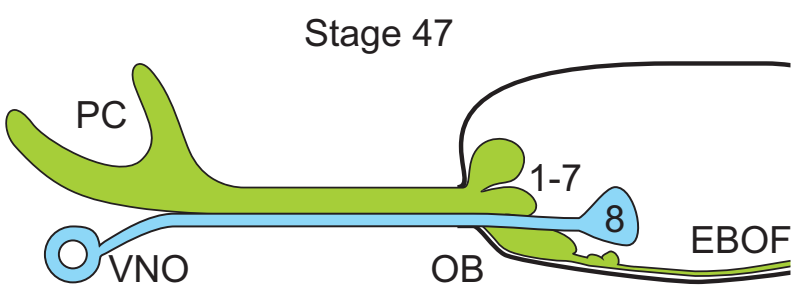

B

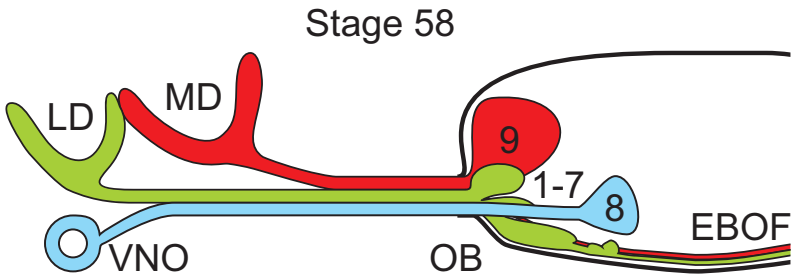

Figure 1.1: Schematic parasagittal sections through the olfactory system of larval Xenopus laevis

A: Fully developed bipartite olfactory system at stage 47 . The olfactory system is segregated into the 'larval water nose' principal cavity (PC) and the vomeronasal organ (VNO). Olfactory sensory neurons expressing class I olfactory receptors project from the principal cavity to the $\mathrm{OB}$, where they form the projection fields PF1 - 7 or leave the OB ventromedially as EBOF. The olfactory sensory neurons from the vomeronasal organ project into PF8 within the accessory OB.

B: Final spatial configuration of the tripartite olfactory system at stage 58 . The olfactory system is segregated into the 'water nose' (LD), the 'air nose' (MD) and the vomeronasal organ. Olfactory sensory neurons expressing class I olfactory receptors project from the LD to the ventral OB and form PF1 - 7. Olfactory sensory neurons expressing class II olfactory receptors project from the MD to the dorsal $\mathrm{OB}$, forming a new projection field PF9. The accessory olfactory system remains unchanged. Some OSN axons from the $\mathrm{LD}$ and the MD leave the $\mathrm{OB}$ as EBOF.

Abbreviations: 1 - 9, PF1 - 9; EBOF, extrabulbar olfactory fibers; OB, olfactory bulb; PC, principal cavity; VNO, vomeronasal organ; LD, lateral diverticulum; MD, medial diverticulum.

Modified and combined from (Reiss and Burd, 1997) and (Gaudin and Gascuel, 2005).

\subsubsection{Development and functional organisation of the olfactory bulb}

The development of the $\mathrm{OE}$ goes along with changes within the $\mathrm{OB}$. The arrival of the first OSN axons in the $\mathrm{OB}$ at stage 30 is followed by the differentiation of the $\mathrm{OB}$ starting at stage 32 (Byrd and Burd, 1991). All layers of the OB (olfactory nerve layer, glomerular layer, mitral cell layer and granule cell layer) become apparent at stage 44 . The coarse organisation of the main $\mathrm{OB}$ becomes indistinguishable from the adult pattern by stage 48 (Byrd and Burd, 1991). 
The general structure of the main OB (Figure 1.2) consists of a series of layers. In the olfactory nerve layer the OSN axons enter the OB and project towards the glomerular layer. The glomerular layer is formed by OSN axon terminals and mitral cell neuropil which aggregate into spherical structures called olfactory glomeruli. The axons from single OSNs bifurcate extensively when approaching the glomerular layer and innervate more than one glomerulus in most cases (Nezlin and Schild, 2005). There are about 350 olfactory glomeruli within the main OB at stages 51 - 56 (Manzini et al., 2007b; Nezlin and Schild, 2000). These olfactory glomeruli lack the distinct borders that are typical for the mammalian olfactory system (Nezlin et al., 2003). The periglomerular cells are scattered within the glomerular layer and their dendrites form extensive arborizations without forming visible glomerular tufts (Nezlin and Schild, 2005). The somata of mitral cells are found throughout the region between the glomerular layer and the granule cells which form a compact group of cells near the paraventricular ependyma (Byrd and Burd, 1991). The mitral cells usually innervate two to three glomeruli with their dendrites (Nezlin and Schild, 2000; Nezlin and Schild, 2005; Nezlin et al., 2003). An antiglutamate staining of the main OB revealed OSN axons, glomerular neuropil and somata of mitral cells (Nezlin and Schild, 2000). Both periglomerular cells and granule cells were identified as GABA immunopositive (Nezlin and Schild, 2000). This corresponds to the assumed functions within the main OB: the olfactory glomeruli contain the first glutamatergic synapse of the olfactory system between OSN axon terminals and mitral cell dendrites; mitral cells are the output neurons towards the olfactory cortex; periglomerular and granule cells act as inhibitory interneurons (Ache and Young, 2005; Ennis et al., 1996).

The changes related to the conversion of the main olfactory system from purely aquatic to the bipartite adult can be seen in the constitution of the primary olfactory projections and were studied in detail by Gaudin and Gascuel (2005): at stage 47 the olfactory projections are grouped into eight projection fields (PF1 - 8). The PF1 7 are formed within the main OB by OSN axons emerging from the principal cavity. Olfactory sensory neuron axons from the vomeronasal organ form PF8 within the accessory OB (Figure $1.1 \mathrm{~A}$ ). At stage 49 (synchronous with class II olfactory receptor expression as described above) an additional projection field PF9 appears in the dorsomedial part of the OB. Both olfactory bulbs start to fuse along the midline in this dorsomedial area at stage 50 (Nieuwkoop and Faber, 1994; Weiss, 1986). Some OSN axons project into the 
contralateral PF9 through this fusion zone (Byrd and Burd, 1991). At stage 58 the PF1 - 7 are innervated exclusively from LD by class I expressing OSN axons and PF9 is innervated only by class II expressing OSN axons from MD (Figure 1.1 B) (Gaudin and Gascuel, 2005; Reiss and Burd, 1997).

The extrabulbar olfactory fibers were first discovered by Szabo et al. (1991) in teleost fish. For Xenopus laevis, EBOF was first described by Hofmann and Meyer (1991a, 1991b) as primary olfactory fibers that bypass the OB and partly cross to the contralateral hemisphere by the anterior commissure. In Xenopus laevis, EBOF

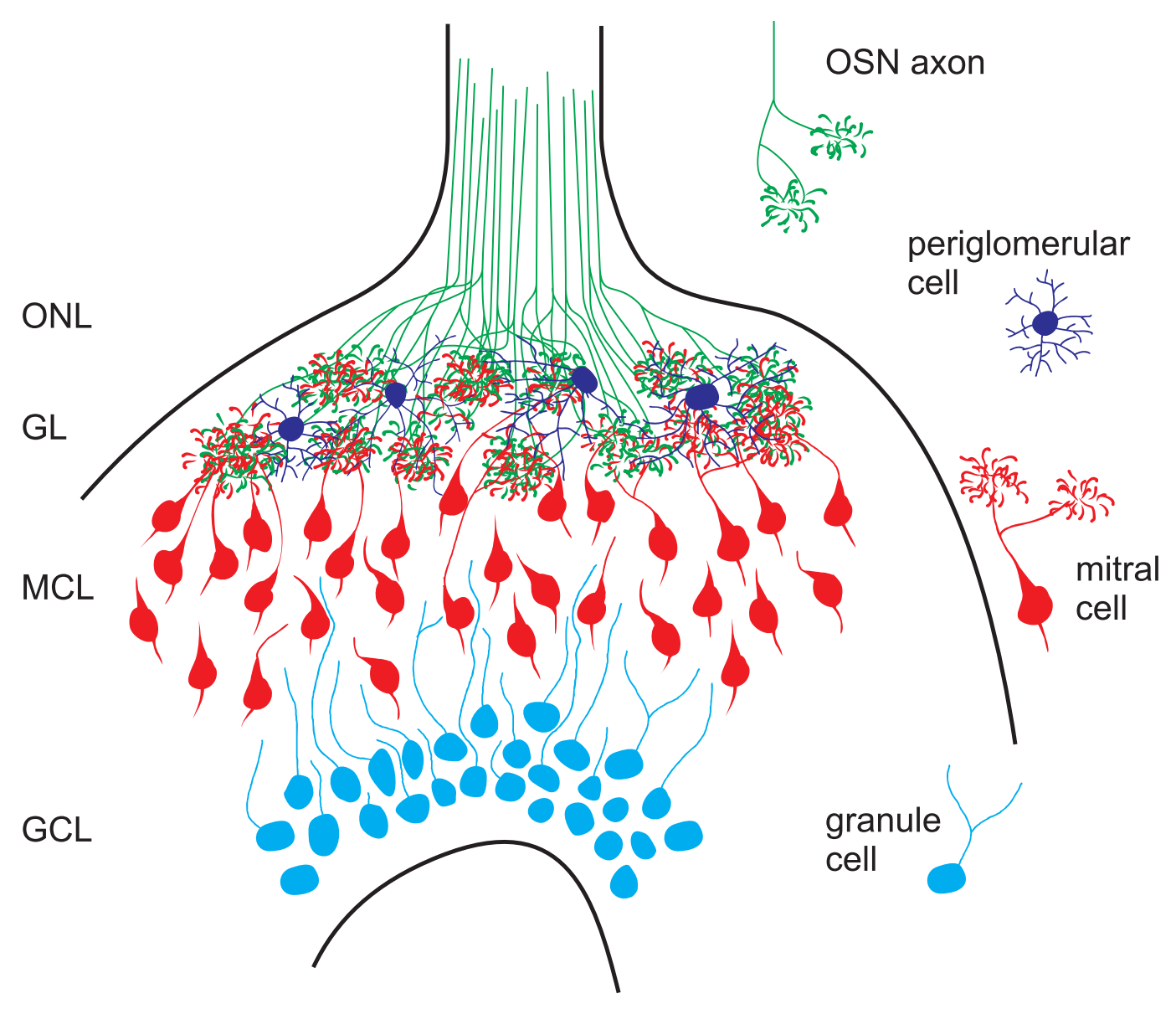

\section{Figure 1.2: Schematic overview of the main olfactory bulb of Xenopus laevis}

Olfactory sensory neuron axons enter the OB through the olfactory nerve and form tufted terminals within the glomerular layer. Periglomerular cells are scattered throughout the glomerular layer. Granule cells lie densely packed near the paraventricular ependyma and extend their dendrites into the mitral cell layer. Mitral cells lie between the glomerular layer and the granule cell layer and innervate several glomerular structures with their dendrites.

Abbreviations: GCL, granule cell layer; GL, glomerular layer; ONL, olfactory nerve layer; OSN, olfactory sensory neuron; MCL, mitral cell layer. 
innervate the ipsilateral preoptic area and bilaterally innervate the hypothalamus (Hofmann and Meyer, 1991a, 1991b; 1992; Pinelli et al., 2004). The extrabulbar olfactory fibers were found in all investigated tadpole stages from stage 45 to adults (Gaudin and Gascuel, 2005; Pinelli et al., 2004). Retrograde tracing identified these fibers as OSN axons. These backtraced OSNs were scattered within the OE without obvious preferential distribution (Pinelli et al., 2004).

\subsubsection{Morphology of the primary olfactory projection in larval Xenopus laevis}

Currently, two different nomenclatures are used for the primary olfactory projection in the main OB of larval Xenopus laevis. The coarse classification is based on the olfactory nerve tracing experiments with biocytin, presynaptic protein distribution and responses to different odorant classes (Manzini et al., 2007b). The more detailed classification is based on the analysis of carbocyanine tracings of the olfactory nerve, soybean agglutinin staining and accounts for the ontogenetic changes related to the separation of the principal cavity into the medial ('air nose') and the lateral ('water nose') diverticulum (Gaudin and Gascuel, 2005). Both classifications are compared in the following.

The classification by Manzini et al. (2007b) differentiates four glomerular clusters within the main $\mathrm{OB}$ which are schematically presented in Figure $1.3 \mathrm{~A}$. The olfactory glomeruli within the ventral part of the OB are grouped into four glomerular clusters. The three major glomerular clusters are named according to their position along the mediolateral axis: lateral cluster, intermediate cluster and medial cluster. The fourth glomerular cluster is reported to contain only about four glomeruli (compared to $71-175$ for major clusters) and is therefore named the 'small cluster'. At the height of the olfactory nerve only the three major clusters are visible. In the dorsal direction the intermediate and medial clusters fuse to a single medial cluster.

The classification of Gaudin and Gascuel (2005) differentiates eight projection fields (PF1 - 7 and PF9) in the main OB and one projection field (PF8) in the accessory OB. The classification of projection fields of the main OB for the stages $52-66$ is presented schematically in Figure 1.3 B. In the ventral part of the main OB three projection fields $(\mathrm{PF} 2-4)$ are visible. The solitary identifiable structures of the PF3 are separately outlined in this schematic. The identifiable structures beta and gamma are presumed to be separate glomeruli in this thesis and are further designated as G1 $\beta$ and Gl $\gamma$. Additionally, the identifiable structure delta (IS $\delta$ ) and EBOF are outlined. In the dorsal 
direction PF2 - 4 are replaced by three following projection fields PF1, PF5 and PF7. More dorsally these three projection fields in turn are replaced with PF6 and the projection field of the medial diverticulum (PF9). PF9 extends over the midline towards the contralateral $\mathrm{OB}$ and fuses with the contralateral PF9. The projection fields at the stages $47-51$ are similar to the projection fields at the stages $52-66$ with the exception of PF9 which does not exist until stage 49 and successively grows in size (Figure 1.1). PF8 belongs to the accessory olfactory system and is therefore not included in this schematic. The identifiable structure alpha is not associated with any projection field and was not included in this schematic either since it disappears after stage 58 .

A

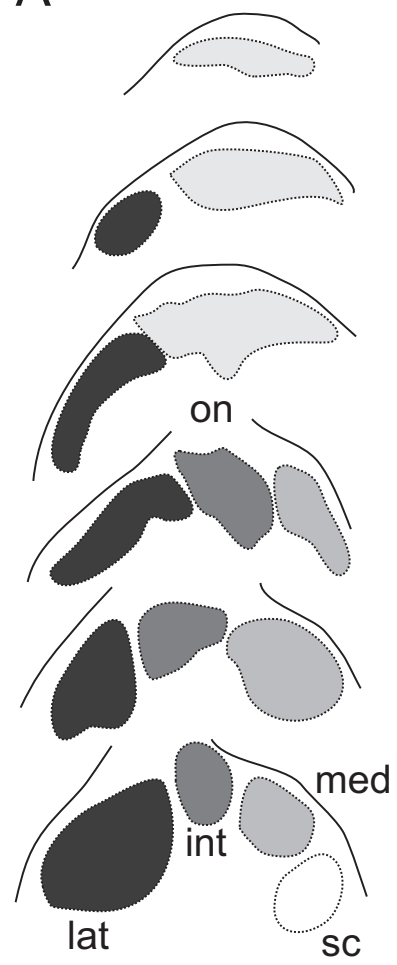

B

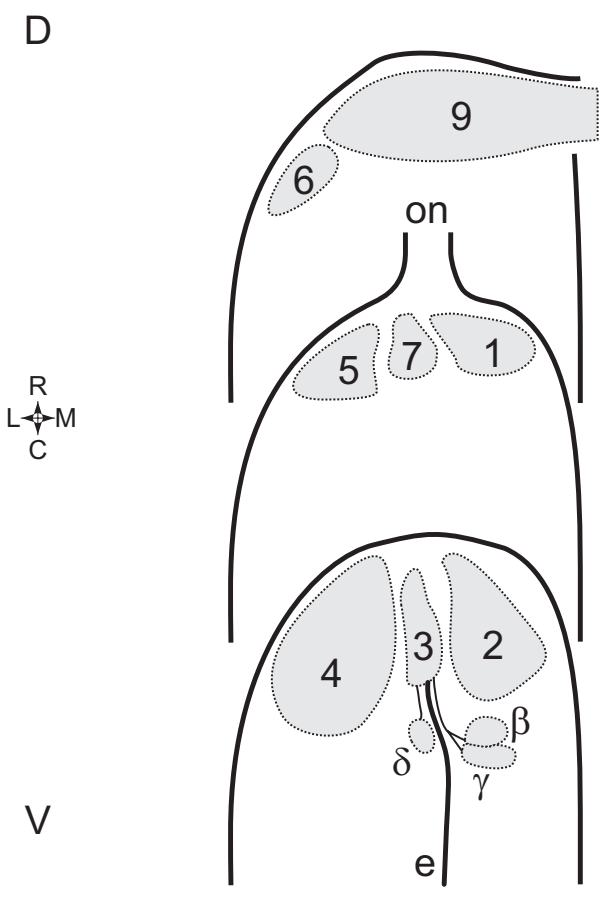

\section{Figure 1.3: Two nomenclatures for primary olfactory projections}

A: Schematic representation of glomerular clusters of the main OB. Transversal sections from dorsal to ventral (bottom section) with borders of the respective glomerular clusters. Adopted from Manzini et al., 2007b.

B: Schematic representation of the projection fields PF1 - 7 and PF9 for stage 49 - 59 tadpoles defined by Gaudin and Gascuel. Adopted from Gaudin and Gascuel, 2005.

Abbreviations: $1-7$, PF $1-7 ; 9$, PF9; $\beta$, Gl $\beta ; \gamma$, Gl $\gamma ; \delta$, IS $\delta$; e, extrabulbar olfactory fibers; on, olfactory nerve; med, medial cluster; int, intermediate cluster; lat, lateral cluster; sc, small cluster; C, caudal; D, dorsal; L, lateral; M, medial; R, rostral; V, ventral. 
The comparison of these two classifications shows that the projection fields of Gaudin and Gascuel (2005) can be consolidated to corresponding glomerular clusters as defined by Manzini et al. (2007b). PF4 - 6 correspond to the lateral cluster. PF3 and PF7 correspond to the intermediate cluster and PF1, PF2 and PF9 correspond to the medial cluster. The small cluster corresponds most likely to the identifiable structures of PF3 which usually lie caudally from PF2 (own observations). The morphological differentiation between lateral and intermediate clusters as introduced by Manzini et al. (2007b) is feasible in the transversal slices, usually used for physiological calcium imaging experiments. Further division into the individual projection fields PF3 - 7 is not feasible. However, the medial cluster consists of the projection fields with different epithelial origin and ontogeny. PF1 and PF2 consist presumably of OSN axons originating from the lateral diverticulum ('water nose'). PF9 is established later (after stage 47) and consists presumably of OSN axons originating from the medial diverticulum ('air nose') (Gaudin and Gascuel, 2005; Meyer et al., 1996). Additionally, intermingling of ipsilateral and contralateral primary olfactory fibers is reported only for PF9 after fusion of both OBs after stage 50 (Byrd and Burd, 1991; Gaudin and Gascuel, 2005). These characteristics legitimate a discrimination of the medial cluster into PF1 and PF2 as ventromedial cluster and PF9 as dorsomedial cluster. A further discrimination between PF1 and PF2 is not feasible for the same reasons as mentioned above. Only when the most ventral part of the OB is within the field of view, the lateral, intermediate and ventromedial clusters can be definitely identified as PF4, PF3 and PF2 respectively. The 'small cluster' presumably corresponds to G1 $\beta$, Gl $\gamma$ and IS $\delta$ of the PF3. This combined and modificated nomenclature will be used for the further description of primary olfactory projection in this study.

\subsection{Bilateral processing in the olfactory system}

In many sensory systems the information from bilateral receptive fields is integrated to extract spatial information. For example, in vision, the binocular perception is used to extract information about depth (Gonzalez \& Perez, 1998) while in audition, interaural phase and intensity differences are used for the localisation of sound sources (King et al., 2001; Konishi, 2000). Also the olfactory system extracts spatial information from bilateral inputs in humans (Porter et al., 2005), rats (Rajan et al., 2006) as well as flies (Duistermars et al., 2009). However, in contrast to the visual and auditory systems, the neural substrates which integrate information from both olfactory epithelia in the 
vertebrate olfactory system are described only fragmentarily. In mouse, the first interhemispheric connection within the olfactory system is relayed through neurons of the anterior olfactory nucleus pars externa (Yan et al., 2008). In anamniotic vertebrates, some mitral cells project directly to the contralateral $\mathrm{OB}$ through the anterior commissure (Eisthen, 1997; Huesa et al., 2000). While these examples represent interhemispheric connections upstream of olfactory glomeruli, it was shown in Xenopus laevis that already the axons of OSNs innervate the contralateral OB (Byrd and Burd, 1991; Gaudin and Gascuel, 2005; Nezlin and Schild, 2005). This might represent a neural substrate for bilateral processing at the level of olfactory glomeruli.

\subsection{Goals of the thesis}

While the existence of OSN axons innervating the contralateral OB is established (Byrd and Burd, 1991; Gaudin and Gascuel, 2005; Nezlin and Schild, 2005), the detailed morphology and the physiological function of this contralateral innervation remain unclear. The first goal of this thesis is to explore the morphology of the contralateral innervation by OSN axons and to investigate whether stimulation of the ipsilateral OE with odorants or alternative qualities such as temperature and mechanical stimuli is processed within the contralateral OB.

A recent study revealed that some OSNs located within the Grüneberg ganglion (Grüneberg, 1973) of mouse respond to cool ambient temperatures (Mamasuew et al., 2008). This observation raised the question whether thermosensitivity is a more general feature of the olfactory system and can be found in an anamniotic vertebrate, such as larval Xenopus laevis. The second goal of this thesis is therefore to examine whether olfactory glomeruli formed by axon terminals of thermosensitive OSNs can be found and whether the response upon temperature stimulation is transmitted through the first olfactory synapse leading to an activation of mitral cells. 


\section{Materials and Methods}

\subsection{Xenopus laevis}

The animal model used for this study is the African clawed frog, Xenopus laevis (Daudin, 1802). Xenopus laevis is an anamniotic vertebrate of the family Pipidae, order Anura of the class Amphibia. It is a well established laboratory animal and is frequently used as an animal model in developmental biology and physiology. Particularly, the olfactory system of Xenopus laevis tadpoles was studied extensively in respect to morphology, development and physiology. Available data on the constitution of the $\mathrm{OE}$ (Hansen et al., 1998; Mezler et al., 1999) and the OB (Byrd and Burd, 1991) are supplemented with physiological studies. These studies range from cellular level (Gliem et al., 2009; Hassenklöver et al., 2008; Manzini and Schild, 2003b; Manzini and Schild, 2004) to behavioural experiments (Altner, 1962). These fundamental studies on the olfactory system of Xenopus laevis deliver insights into the functioning of the vertebrate olfactory system, thereby providing a suitable model system for further studies.

The tadpoles used for the experiments in this thesis were bred in the laboratory. The adult animals were bought from a commercial supplier or raised in house. The breeding was artificially induced by human chorionic gonadotropin injections for both male and female animals to initiate amplexus and egg laying.

For experiments, tadpoles of developmental stages 47 - 53 were used. These were classified according to Nieuwkoop and Faber (1994). All experiments with Xenopus laevis tadpoles were conducted according to guidelines approved by the Göttingen University Committee of Ethics in Animal Experimentation.

\subsection{Fluorescence imaging setup and dyes}

\subsubsection{Fluorescence imaging}

Fluorescence is a physical property of molecules to absorb energy of a specific excitation wavelength and re-emit part of this energy as a photon of lower wavelength. Fluorescence microscopes detect fluorescence of the biological tissue itself (autofluorescence) or specific fluorescent dyes. The optical pathway of a fluorescence microscope comprises an excitation and an emission pathway. The excitation pathway consists of an excitation light source (usually a laser or a xenon arc lamp), a spectral 
band pass filter and a dichroic mirror which reflects the excitation light to the objective's back aperture. The objective focuses the light onto the sample. The fluorophore (the fluorescent part of the molecule) within the sample is excited by the absorption of a photon. Following thermal relaxation it emits a photon at a lower wavelength that can pass the dichroic mirror in the emission pathway. The emitted light is filtered through an optical filter to block the remaining excitation light and is detected with a light sensor (e.g. a photomultiplier or a Charge-coupled Device (CCD) chip). The optical pathways can be modified for the use of different fluorophores at the same time. In the excitation pathway different excitation wavelengths can be used. The emission pathway can contain additional dichroic mirrors that reflect the emitted light from different fluorophores to different detectors. The utilisation of several emission pathways can lead to a 'bleed-through' artefact caused by the incomplete separation of overlapping emission spectra. This artefact is usually avoided by choosing fluorophores with clearly separated emission spectra and a consecutive acquisition of the single channels. The residual 'bleed-through' was corrected by spectral unmixing as described in image processing (section 2.6.3).

\subsubsection{Confocal laser-scanning microscope setup}

In this study a confocal laser-scanning microscope (CLSM) was used for fluorescence imaging experiments. This CLSM consisted of an inverted microscope (Axiovert 100M) with an attached laser scanning unit (LSM 510). The configuration of the optical pathways for the different combinations of dyes as well as the fluorophores used are described in the following section.

\subsubsection{Optical pathways and fluorophores}

The fluorophores Alexa Fluor 488 and Alexa Fluor 568 are optimised for low photobleaching and high quantum yield at low excitation intensities. Alexa Fluor 488 (excitation maximum at $\lambda_{\mathrm{ex}}=499 \mathrm{~nm}$, emission maximum at $\lambda_{\mathrm{em}}=519 \mathrm{~nm}$ ) was excited with a monochromatic laser light, $\lambda=488 \mathrm{~nm}$. The emission filter was a $505-530 \mathrm{~nm}$ bandpass filter. Alexa Fluor $568\left(\lambda_{\mathrm{ex}}=579 \mathrm{~nm}, \lambda_{\mathrm{em}}=603 \mathrm{~nm}\right)$ was excited with a monochromatic laser light, $\lambda=543 \mathrm{~nm}$. The emission filter was a $585 \mathrm{~nm}$ longpass filter. Both fluorophores were used for the morphological imaging of OSN axons.

Fluo-4 and Fura Red are calcium sensitive fluorophores. Calcium sensitive fluorophores change their excitation or emission spectrum when binding to calcium. Consequently, a 
change in fluorescence intensity indicates calcium binding. In the present study the qualitative $\left[\mathrm{Ca}^{2+}\right]_{\mathrm{i}}$ transients were used to detect neuronal activity. A quantification of $\left[\mathrm{Ca}^{2+}\right]_{\mathrm{i}}$ within these neurons was not performed. Fluorescence intensity changes were normalised to the fluorescence intensity at the start of the measurement and are presented either as $\Delta \mathrm{F} / \mathrm{F}$ traces or as spatial maps (see section 2.6.1 for details).

Fluo-4 has a low basal fluorescence and a very high increase in fluorescence intensity upon binding to calcium (Gee et al., 2000). Fluo-4 $\left(\lambda_{\mathrm{ex}}=494 \mathrm{~nm}, \lambda_{\mathrm{em}}=516 \mathrm{~nm}\right)$ was excited with a monochromatic laser light, $\lambda=488 \mathrm{~nm}$. The emission filter was a $505 \mathrm{~nm}$ longpass filter when used as the only fluorescence dye. When used in combination with a second dye, the emitted light was filtered with a $505-530 \mathrm{~nm}$ bandpass filter.

Fura Red has a large stokes shift $\left(\lambda_{\mathrm{ex}}=458 \mathrm{~nm}, \lambda_{\mathrm{em}}=597 \mathrm{~nm}\right)$ and shows a significant decrease of fluorescence upon calcium binding. This fluorescence decrease makes Fura Red a valuable tool for visualising vital cells which have a low basal $\left[\mathrm{Ca}^{2+}\right]_{\mathrm{i}}$ (Manzini and Schild, 2003a). This dye was excited with a monochromatic laser light, $\lambda=488 \mathrm{~nm}$. The emission filter was a $585 \mathrm{~nm}$ longpass filter.

These two calcium sensitive fluorophores were used for different purposes. Fluo-4 was used to detect short $\left[\mathrm{Ca}^{2+}\right]$ transients within neurons that occurred as a response during stimulation. Fura Red was used to visualise the morphology of neurons.

\subsection{Dye loading procedures}

\subsubsection{Electroporation loading procedure}

The application of an electric field over the cell membrane during electroporation induces pores permeable for macromolecules, which last for $50 \mathrm{~ms}$ to several seconds (Teruel and Meyer, 1997) within this membrane. The electroporation procedure has been successfully used for in vivo loading of a large repertoire of macromolecules (DNA (Boutin et al., 2008), antisense morpholinos (Falk et al., 2007) and dextran bound dyes (Bonnot et al., 2005; Nagayama et al., 2007)). The electroporation loading is possible at different scales, from single cells (Bestman et al., 2006; Nevian and Helmchen, 2007) to entire brains of Xenopus laevis tadpoles (Haas et al., 2002). The survival of the electroporated cells greatly depends on the strength of the electric field applied, the duration of the voltage pulses and the number of repetitions. A detailed study of single cell electroporation reports a membrane potential recovery of 
electroporated cells within a minute after successful electroporation (Nevian and Helmchen, 2007).

In this study the OSNs were loaded with a dye using electroporation in the nasal cavities. The loading conditions used were determined empirically to cause a sufficient loading without noticeable damage to the tissue during the electroporation. A minimal time of one day between electroporation and imaging of the OB was sufficient to allow a recovery of the OSNs. All dyes used for electroporation consisted of fluorophores conjugated to $10 \mathrm{kDa}$ dextran. Dextrans are biologically inert, hydrophilic polysaccharides used as carriers during long term tracing experiments. The dextran conjugation prevents an extrusion of the fluorophore (Kreitzer et al., 2000). After electroporation loading of OSNs in the OE, the dextran-conjugated dye is transported by axonal anterograde transport through the olfactory nerve towards the OSN axon terminals in the $\mathrm{OB}$.

The electroporation loading was performed using a custom-built device. This device contained a square pulse generator (Isolated Stimulator, Type 2533) with adjustable voltage (maximal $100 \mathrm{~V}$ ) and pulse length. This current source was connected to two platinum wire electrodes of $250 \mu \mathrm{m}$ diameter placed in $3 \mathrm{~mm}$ distance. The distance between the electrodes was slightly adjusted to fit into both nasal cavities of the tadpole prior to electroporation. For electroporation, Xenopus laevis tadpoles were anaesthetised with $0.02 \%$ MS-222 for at least one minute until all movement ceased. The anaesthetised animals were transferred to a silicone-covered petri dish and water was partially soaked up from their nasal cavities using tissue paper. Small crystals $(1-10 \mu \mathrm{g})$ of $10 \mathrm{kDa}$ dextran-conjugated dye were placed into the nasal cavity. These crystals dissolved within one minute. The platinum electrodes were then inserted into both nasal cavities without damaging the OE tissue. Twelve $30 \mathrm{~V}$ square pulses of $20 \mathrm{~ms}$ duration were applied with alternating polarity. Each pulse was manually triggered at about $1 \mathrm{~Hz}$. The animals were placed back into an aquarium and kept at low light and under otherwise normal conditions for one to five days prior to the experiments. At least one day was required for sufficient anterograde axonal transport of the $10 \mathrm{kDa}$ dextran to the axon terminals in the $\mathrm{OB}$. 


\subsubsection{Loading of cells with AM coupled dyes}

Bulk loading of cells with acetoxymethyl (AM) coupled dyes is a staining technique frequently used. Fluorescent dyes can be coupled to AM by their carboxylic acid groups. This masks the charges of the dye, makes them lipophilic and thus membrane permeable. When applied in the extracellular solution these AM coupled dyes cross the cell membrane and enter the cytosol. Inside the cells the AM ester groups are cleaved by non-specific esterases and the dye molecule becomes charged. This prevents the dye molecule from leaving the cell by crossing the cell membrane again. While the deesterificated, charged dye is trapped inside the cell, more AM coupled, lipophilic dye molecules enter the cell and are consequently de-esterificated. This process can lead to a dye accumulation that exceeds the extracellularly applied concentration. The intracellular dye accumulation might be reduced by mechanisms that extrude xenobiotics (Jakob et al., 1998). In the OE of Xenopus laevis (and to a lesser extent in OB neurons) staining with AM coupled dyes can be improved by blocking multidrug resistance transporters with MK571 (Gekeler et al., 1995; Manzini and Schild, 2003a; Manzini et al., 2008).

The staining with AM coupled dyes can be achieved by bath incubation or bolus loading. Bath incubation was used to load cells at tissue surfaces of sliced tissue. Bolus loading was performed by pressure-injecting the AM coupled dye solution through a patch-pipette deep into intact tissue. The staining procedures and the dye solutions mentioned here are described in detail in section 2.4.

\subsection{Tissue preparation}

The preparations were conducted after anaesthetizing Xenopus laevis tadpoles in a mixture of water and ice and a subsequent decapitation. A rectangular block containing the forebrain and nose was cut out of the head leaving the OBs, the olfactory nerves and the olfactory epithelia undamaged. This block was used for the following, experimentspecific preparation.

\subsubsection{Sliced preparation and bath incubation staining}

A sliced preparation containing both the nose and the OB ('sliced nose-olfactory bulb preparation') was performed as described by Manzini et al. (Manzini et al., 2002). The tissue block was glued to a stage of a vibroslicer (VT 1000S) and covered with Ringer solution. For imaging of the $\mathrm{OB}$, the dorsal part of the OB was cut just dorsal from the 
olfactory nerve with the first cut. For imaging of the $\mathrm{OE}$, the first cut was performed at the middle level of the olfactory mucosa. In both cases (OB or OE imaging), a second cut was performed about $200 \mu \mathrm{m}$ deeper (without damaging the olfactory nerve) to create a second plain surface. This preparation was transferred to a recording chamber containing Ringer solution.

The sliced preparations were stained by bath incubation with AM coupled dyes. The acetoxymethyl coupled dye was dissolved in DMSO containing $20 \%$ Pluronic F-127. This solution was mixed with Ringer solution to reach the final bath solution concentration of $50 \mu \mathrm{M}$ dye/AM, $0.5 \%$ DMSO. To improve the dye uptake, multidrug resistance transporters were specifically blocked by adding $50 \mu \mathrm{M}$ MK571 to the bath solution. The slice was incubated for 30 minutes in this bath solution and was perfused with Ringer solution for at least 20 minutes before imaging experiments.

\subsubsection{Explanted nose-olfactory bulb preparation and bolus loading}

For the 'explanted nose-olfactory bulb preparation', the block with the olfactory system was placed in a silicon-covered petri dish with the ventral side up. The block was fixed with two fine needles pierced between the olfactory nerves and covered with a drop of Ringer solution. Cartilaginous tissue ventral of the telencephalon was cut away (with fine scissors under the binocular) to expose the OBs. This explant was transferred to the recording chamber.

\subsubsection{Bolus loading}

Bolus loading of AM coupled dyes was performed with explanted nose-olfactory bulb preparations at an upright microscope (Axioskop 2). The microscope was equipped with a patch pipette holder coupled to a micromanipulator and an air pressure device. Patchpipettes were pulled from borosilicate glass capillaries $(1.8 \mathrm{~mm}$ outer diameter) with a two stage electrode puller. The concentration of AM dye in the Ringer solution for bolus loading was tenfold higher than that for bath incubation (500 $\mu \mathrm{M}$ AM dye, $5 \%$ DMSO, $1 \%$ Pluronic F-127, $500 \mu \mathrm{M}$ MK571). A patch pipette (resistance 5-8 M 2 , tip diameter $1-2 \mu \mathrm{m}$ ) was filled with $6 \mu \mathrm{l}$ of this solution. Air pressure of $50-300 \mathrm{hPa}$ was applied to the patch-pipette to avoid clogging of the pipette tip. The patch pipette was carefully inserted into the ventral $\mathrm{OB}$ at the olfactory nerve layer and pushed dorsocaudally until the mitral cell layer. Slight displacements of the tissue caused by the outstream of solution from the pipette tip were monitored to assure a continuous 
injection. After reaching the intended injection depth, the outflow of the dye solution was continued for one to six minutes. The pipette was retracted and used for the injection of the second OB. Eventually, the explant was left in Ringer solution for 30 minutes to allow uptake and intracellular de-esterification of the AM coupled dye.

\subsubsection{Tissue fixation and histochemistry}

Histochemistry was performed with OBs from tadpoles that had previously been electroporated with biocytin. The electroporation with biocytin was performed in the same way as described previously for $10 \mathrm{kDa}$ dextrans. Biocytin is a much smaller molecule $(372 \mathrm{Da})$ than the $10 \mathrm{kDa}$ dextrans and therefore reaches the OSN axon terminals in the OB faster. Xenopus laevis tadpoles that had been electroporated with biocytin within their nasal cavities were anaesthetized and killed (as described above) two hours later. A tissue block containing the OB was cut out and fixed in $4 \%$ paraformaldehyde over night at $4{ }^{\circ} \mathrm{C}$. The fixed tissue was washed in phosphate buffered saline (PBS) for 15 minutes, embedded in $5 \%$ low melting point agarose and vibratome sectioned at $70 \mu \mathrm{m}$. The sections were washed in PBS containing $0.2 \%$ Triton X-100 (PBST) for 15 minutes. Subsequently, the sections were incubated with Alexa Fluor 488 conjugated avidin $(100 \mu \mathrm{g} / \mathrm{ml}$ in PBST) for two hours at room temperature. The glycoprotein avidin binds biocytin with high selectivity and affinity $\left(\mathrm{Kd} \sim 10^{-15} \mathrm{M}\right)$ and thereby links the conjugated fluorophore to biocytin (Heitzmann and Richards, 1974). The sections were washed at least three times for 15 minutes with PBS. The sections were then transferred to a solution consisting of $60 \%$ glycerol/PBS, following $80 \%$ glycerol/PBS (one hour each) and finally mounted on glass slides.

\subsection{Stimulation of the olfactory epithelium}

The sliced and explanted preparations of the stained olfactory system were imaged with an inverted microscope and were positioned accordingly. The explanted nose-olfactory bulb preparation was placed with its previously exposed ventral OB facing the cover slip. The sliced nose-olfactory bulb preparation and the sliced OE preparation were positioned with their first sliced surface facing the cover slip. The stimuli were applied through a custom-built funnel applicator (Schild, 1985), the outlet of which was placed in front of the OE. The experimental chamber was constantly perfused with Ringer solution $(2.4 \mathrm{ml} / \mathrm{min})$. The stimulus solution was applied through an electronic pipette (HandyStep electronic). This electronic pipette was modified so that it released the 
stimulus solution upon arrival of a transistor-transistor logic (TTL) trigger from a custom-built control device. The control device also triggered the start of the image acquisition. The constant perfusion of the OE with Ringer solution was not interrupted during the release of the stimulus solution into the funnel. The pressure changes at the outlet were minimized by a funnel overflow as described by Schild (1985). The time interval between the TTL trigger from its control device and the arrival of the stimulus solution at the OE consisted of two delays. The first delay was the time between the arrival of the TTL signal at the electronic pipette and the start of the release of the stimulus solution. The second delay was the time between the release of the stimulus solution in the funnel and the outflow of the stimulus solution through the outlet in front of the OE. The sum of both delays was measured by imaging a fluorescein-containing solution as dummy stimulus. The imaged region was placed directly at the funnel outlet for this measurement. The time between the TTL trigger release and arrival of the fluorescein solution at the outlet of the funnel was $2.5 \pm 0.2$ seconds (mean \pm standard deviation). This delay was taken into account when representing the stimulus application in a figure.

\subsubsection{Stimulation with odorants}

Odorant stimulation was performed by releasing Ringer solution with dissolved aquatic odorants with the electronic pipette into the funnel. The odorant solutions are listed in section 2.8.1.

\subsubsection{Temperature stimulation}

Ringer solution heated (up to $50{ }^{\circ} \mathrm{C}$ ) or cooled (down to $0{ }^{\circ} \mathrm{C}$ ) was used as temperature stimulation. The Ringer solution applied as temperature stimulus was always aliquoted from the same bottle as the constantly perfused Ringer solution to avoid any artefacts caused by concentration differences. The temperature of the Ringer solution pipetted into the funnel was measured in advance (abbreviated as $T_{R}$ ) of the stimulation experiment. It was kept constant between the measurements to assure the reproducibility of experiments with the same temperature. The stimulus Ringer solution was placed on ice to attune the temperature to $0{ }^{\circ} \mathrm{C}$. For other temperatures adjustable heating-cooling units were used.

A thermocouple (NiCr-Ni) was placed between the outlet of the funnel applicator and the OE. This thermocouple measured the temperature differences at the OE caused by 
the Ringer solution of a certain temperature. It consisted of a twisted pair of teflon insulated thermowell wires (diameter $0.2 \mathrm{~mm}$ ). The response time of this thermocouple was 0.3 seconds according to the manufacturer. Temperatures measured at the $\mathrm{OE}$ were read out every second using datalogger (K202) and imported to MATLAB for further analysis. This temperature measurement at the OE was necessary because the temperature difference of the stimulus solution to the room temperature was reduced significantly within the application system. The pipetted Ringer solution mixed with the constantly perfused Ringer solution within the funnel. Additionally, some differences between the stimulus solution temperature and the room temperature decreased on the way towards the OE due to the contact with the metallic funnel. For these reasons, the measured temperature of the pipetted solution can only be considered as an upper estimate for the actual temperature difference at the OE.

The temperature measured directly at the $\mathrm{OE}$ with the thermocouple gives a lower estimate for the actual temperature difference at the OE because of the thermal inertia of the thermocouple. The actual temperature at the OE after applicating the stimulus Ringer solution lies between the temperature of the stimulus solution (that was measured in advance) and the temperature measured by the thermocouple. Hence, in the results section both temperatures were stated for a complete description of a temperature stimulation experiment.

Temperature differences caused by temperature stimulation at the OE were calculated as $\Delta \mathrm{T}=\mathrm{T}(\mathrm{t})-\mathrm{T}_{0}$, with $\mathrm{T}_{0}$ being the temperature measured at the beginning of the experiment. The peak of $\Delta \mathrm{T}$ (abbreviated as $\Delta \mathrm{T}_{\mathrm{p}}$ ) for an experiment was calculated as the maximum of $\Delta \mathrm{T}$ for warm temperature stimulation experiment and as the minimum of $\Delta \mathrm{T}$ for cold temperature stimuli. $\Delta \mathrm{T}_{\mathrm{p}}$ is presented as mean and standard deviation for repetitive stimulations with the same $T_{R}$.

\subsubsection{Mechanical stimulation}

The mechanical stimulus consisted of a complete stop of the Ringer perfusion and a subsequent restart of the perfusion at the previous speed. This was suitable as mechanical stimulus for two reasons. First, the strength of this mechanical stimulus was in the physiological range because it was caused by the solution flow that is otherwise constantly applied. Second, the time course of this stimulus is well defined and easily reproducible. The complete stop and restart of the perfusion were performed by manual 
closing or opening a valve and were registered by the image acquisition software using a trigger from the custom control device.

\subsection{Image processing}

Image processing was done in MATLAB. The acquired images were imported into MATLAB using the DIPimage toolbox. The analysis of images in the MATLAB environment was performed with MATLAB functions, the DIPimage toolbox and matVis. matVis was written by Stephan Junek, Department of Neurophysiology and Cellular Biophysics, University of Göttingen.

\subsection{1 $\Delta \mathrm{F} / \mathrm{F}$ calculations}

Odor-induced changes in $\left[\mathrm{Ca}^{2+}\right]_{i}$ were represented as $\Delta \mathrm{F} / \mathrm{F}$. The detector background $\left(\mathrm{F}_{\mathrm{B}}\right)$ was measured by averaging images acquired using the same settings but without laser light excitation. The basal fluorescence for every pixel at the beginning of the experiment $\left(\mathrm{F}_{0}\right)$ was calculated as the mean of the first ten time points prior to stimulation. $\Delta \mathrm{F} / \mathrm{F}$ was then calculated for every pixel as $\Delta \mathrm{F} / \mathrm{F}=\left(\mathrm{F}(\mathrm{t})-\mathrm{F}_{0}\right) /\left(\mathrm{F}_{0}-\mathrm{F}_{\mathrm{B}}\right)$. The $\Delta \mathrm{F} / \mathrm{F}$ traces for regions of interest (ROIs) were calculated as the mean value of all pixel fluorescence intensities within the ROI. All $\Delta \mathrm{F} / \mathrm{F}$ spatial maps were Gauss-filtered (width $=1$ pixel for the spatial dimensions). Odor responses are shown for the time point where the response peaked.

\subsection{2 'Pixel correlation map'}

A 'pixel correlation map' was calculated by cross-correlating the fluorescence time course of each pixel to the fluorescence time course of its adjacent pixels (Manzini et $a l ., 2007 \mathrm{a})$. The resulting correlation coefficients were then averaged and displayed for every pixel giving a map of correlation coefficients. The image regions containing coherent signals within several pixels were highlighted and pixels with uncorrelated signals were shaded. Since the cross-correlation algorithm used to calculate the 'pixel correlation map' is not sensitive to the actual time point of the transient, the map depends both on spontaneous activity as well as on responses to stimulation. Thus the temporal information within the complete time series was used to calculate a spatial distribution of coherent signals. 


\subsubsection{Spectral unmixing}

The 'bleed-through' artefact could be avoided almost completly by choosing appropriate imaging conditions. Only scans with Alexa Fluor 488 and Fura Red fluorophores caused considerable 'bleed-through' because both were excited at $488 \mathrm{~nm}$ and have overlapping emission spectra. The Alexa Fluor 488 fluorophore showed considerable signal above $585 \mathrm{~nm}$ (red channel), while the emission of Fura Red was restricted to the red channel and did not contribute to the green channel $(505-530 \mathrm{~nm})$.

For spectral unmixing, specimens separately stained with only one of the dyes each were prepared and imaged under the same conditions as the double stained specimens. The detector background was measured without excitation light for both channels and subtracted from all measurements in the first step. The scan of the specimens electroporated with Alexa Fluor 48810 kDa dextran contained signals in both channels, while no signal from the specimens stained with Fura Red in the green channel was detected. The mean signal from both channels of the specimens stained with Alexa Fluor 488 was used to calculate the 'bleed-through' ratio for the Alexa Fluor 488 fluorophore $\left(\mathrm{R}_{\mathrm{g}}\right)$. The amount of the Alexa Fluor 488 signal in the red channel from images of double stained specimens was calculated by multiplying the green channel from the image of double stained specimens with $\mathrm{R}_{\mathrm{g}}$. This product was then subtracted from the red channel to remove the 'bleed-through' of Alexa Fluor 488.

\subsection{Image presentation}

All olfactory bulb images are presented such that the mediolateral axis is oriented from right (medial) to left (lateral). In cases where the imaged OB was in the right hemisphere of the brain, the images were mirrored horizontally for presentation. The allocation of fluorescence signals from specimens electroporated with Alexa Fluor 488 (in the left OE) and Alexa Fluor 568 (in the right OE) to the red or green channel of the image was chosen depending on the side of the OB imaged, thus independent of dye emission spectrum. The traced axons from ipsilateral $\mathrm{OE}$ are presented in green, the axons emerging from the contralateral $\mathrm{OE}$ are presented in red.

Orthogonal maximum intensity projections (MIPs) were calculated for the presentation of imaged volumes. The dorsoventral view was calculated as MIP in the dorsoventral axis, the lateral view was calculated as MIP in the mediolateral direction and the frontal view was calculated as MIP along the rostrocaudal direction. 


\subsection{Materials}

\subsubsection{Solutions}

The Ringer solution used contained $98 \mathrm{mM} \mathrm{NaCl}, 2 \mathrm{mM} \mathrm{KCl}, 1 \mathrm{mM} \mathrm{CaCl}, 2 \mathrm{mM}$ $\mathrm{MgCl}_{2}, 5 \mathrm{mM}$ glucose, $5 \mathrm{mM}$ Na-pyruvate, $10 \mathrm{mM}$ HEPES solved in Milli-Q purified water. It was adjusted to $\mathrm{pH} 7.8$. Its osmolarity was $230 \mathrm{mOsmol} \mathrm{l}^{-1}$.

Odorant solutions were prepared in Ringer solution and stored at $4{ }^{\circ} \mathrm{C}$ for a maximum of five days.

The amino acids mixture contained L-alanine, L-arginine, L-asparagine, L-aspartat, L-cystein, L-glutamat, L-glutamine, L-glycine, L-histidine, L-isoleucine, L-leucine, L-lysine, L-methionine, L-phenylalanine, L-proline, L-serine, L-threonine, L-tryptophan and L-valine at $200 \mu \mathrm{M}$ each. L-tyrosine was not in the mixture because of its low water solubility.

The amines mixture contained 2-phenylethylamine, tyramine, butylamine, cyclohexylamine, hexylamine, 3-methylbutylamine, N,N-dimethylethylamine, 2-methylbutylamine, 1-formylpiperidine, 2-methylpiperidine, N-ethylcyclohexylamine, 1-ethylpiperidine and piperidine at $200 \mu \mathrm{M}$ each.

The Altner alcohol mixture contained beta-ionone, citral, beta-phenylethyl alcohol and gamma-phenylpropyl alcohol at $200 \mu \mathrm{M}$ each.

The $\mathrm{CO}_{2}$ stimulation solution was made of Ringer solution bubbled with pure $\mathrm{CO}_{2}$ gas for 15 minutes prior to application.

Forskolin $(50 \mu \mathrm{M})$ was used as pseudostimulus for OSNs that respond to cAMP mediated stimuli (Manzini and Schild, 2003b).

The bile acids mixture was made from a crude preparation of taurocholic acid from ox bile containing $200 \mu \mathrm{M}$ taurocholic acid and traces of glycocholic, deoxycholic and other bile acids.

The PBS contained $137 \mathrm{mM} \mathrm{NaCl}, 2.7 \mathrm{mM} \mathrm{KCl}, 8 \mathrm{mM} \mathrm{Na}_{2} \mathrm{HPO}_{4}, 1.4 \mathrm{KH}_{2} \mathrm{PO}_{4}$ solved in Milli-Q purified water. It was adjusted to $\mathrm{pH}$ 7.4. 


\subsubsection{List of materials}

\begin{tabular}{ll} 
Adult Xenopus laevis & Kaehler \\
Axioskop 2 & Zeiss \\
Borosilicate glass capillary & Hilgenberg \\
Datalogger K202 & Conrad \\
DIPimage & The Quantitive Imaging Group of TU Delft \\
Electrode puller & Narishige \\
HandyStep electronic & Brand \\
Isolated Stimulator Type 2533 & Digitimer \\
LSM 510/Axiovert 100M & Zeiss \\
MATLAB & Mathworks \\
Mikroliterzentrifuge Biofuge fresco & Kendro Laboratory Products \\
Milli-Q & Millipore \\
NiCr-Ni thermocouple, GTF 300 & Greisinger Elektronik \\
Patch-clamp amplifier EPC7 & List \\
Preparation instruments & Fine Science Tools \\
Thermomixer comfort & Eppendorf \\
Vibroslicer VT 1000S & Leica \\
\hline
\end{tabular}




\subsubsection{List of suppliers}

Alexis Biochemicals

Lörrach

Germany

Brand

Wertheim

Germany

Conrad

Hirschau

Germany

Digitimer

Welwyn GC

UK

Eppendorf

Hamburg

Germany

Fine Science Tools

Heidelberg

Germany

Greisinger Elektronik

Regenslauf

Germany

Hilgenberg

Malsfeld

Germany

Invitrogen

Karlsruhe

Germany

Kendro Laboratory Products

Langenselbold

Germany

Leica Microsystems

Bensheim

Germany

List

Darmstadt

Germany

Mathworks

Natick, MA

USA

Merck

Darmstadt

Germany

Millipore

Schwalbach

Germany

Narishige

Tokyo

Japan

Sigma

Deisenhofen

Germany

The Quantitive Imaging Group of TU Delft

Delft

Netherlands

Zeiss

Jena

Germany 


\subsubsection{List of chemicals}

All common chemicals not included in the list were purchased from Sigma or Merck.

Chemical

Alexa Fluor 48810 kDa dextran

Alexa Fluor 488 avidin conjugate

Alexa Fluor 56810 kDa dextran

Biocytin ( $\varepsilon$-biotinoyl-L-lysine)

Chorionic gonadotropin, human

Fluo-4 10kDa dextran $\left(\mathrm{K}_{\mathrm{d}} \sim 600 \mathrm{nM}\right)$

Fluo-4/AM

Fura Red/AM

MK571

MS-222 (Tricaine)

Pluronic F-127 20 \% solution in DMSO

Taurocholic Acid from ox bile
Product number Supplier

D-22910 Invitrogen

A-21370 Invitrogen

D-22912 Invitrogen

B-1592 Invitrogen

CG10-1VL Sigma

F-36250 Invitrogen

F-14210 Invitrogen

F-14219 Invitrogen

Alexis Biochemicals

A5040

Sigma

P-3000MP Invitrogen

T-0750 Sigma 


\section{Results}

The results are divided into three chapters. In the first chapter, the morphology of the OSN axon projections within the OB is presented. The main focus in this chapter is set on OSN axons that project to the respective contralateral OB. In the second chapter the responses of OSN axons within the OB to odorants, temperature and mechanical stimulation of the OE are presented. The third chapter deals with responses of mitral cells to temperature stimulation of the OE.

\subsection{Morphology of the contralateral projecting axons of olfactory sensory neurons}

In adult Xenopus laevis an innervation of the OB by fibers emerging from the contralateral OE was first reported by Ebbesson et al. (1986). It was also noted in PF9 of larval Xenopus laevis (Byrd and Burd, 1991; Gaudin and Gascuel, 2005; Nezlin and Schild, 2005). A detailed study on the amount of the contralateral innervation and its distribution within different glomerular clusters of Xenopus laevis tadpoles at stage 47 and 52 was performed in this thesis.

To distinguish between the ipsi- and contralaterally projecting OSN axons, Xenopus laevis tadpoles were electroporated with different fluorophores: the left nasal cavity was electroporated with Alexa Fluor $48810 \mathrm{kDa}$ dextran, while the right one was electroporated with Alexa Fluor $56810 \mathrm{kDa}$ dextran. The olfactory bulbs of the electroporated tadpoles were explanted and imaged from the ventral side using the confocal laser scanning microscope.

\subsubsection{The olfactory bulb is innervated by the contralateral olfactory epithelium at stage 47}

A surprising result of the tracing experiments was that already at stage 47 OSN axons were found in the contralateral OB. To date, various studies reported contralateral innervation of the OB by OSN axons only after stage 50 (Byrd and Burd, 1991; Gaudin and Gascuel, 2005; Nezlin and Schild, 2005). Starting at stage 50 both OBs fuse along the midline and thus establish a pathway for OSN axons to the contralateral OB (Nieuwkoop and Faber, 1994; Weiss, 1986). Contrary to these previous studies, OSN axons innervating the contralateral $\mathrm{OB}$ at stage 47 follow the extrabulbar olfactory 
pathway and enter the contralateral hemisphere through the anterior commissure as shown in Figure 3.1.
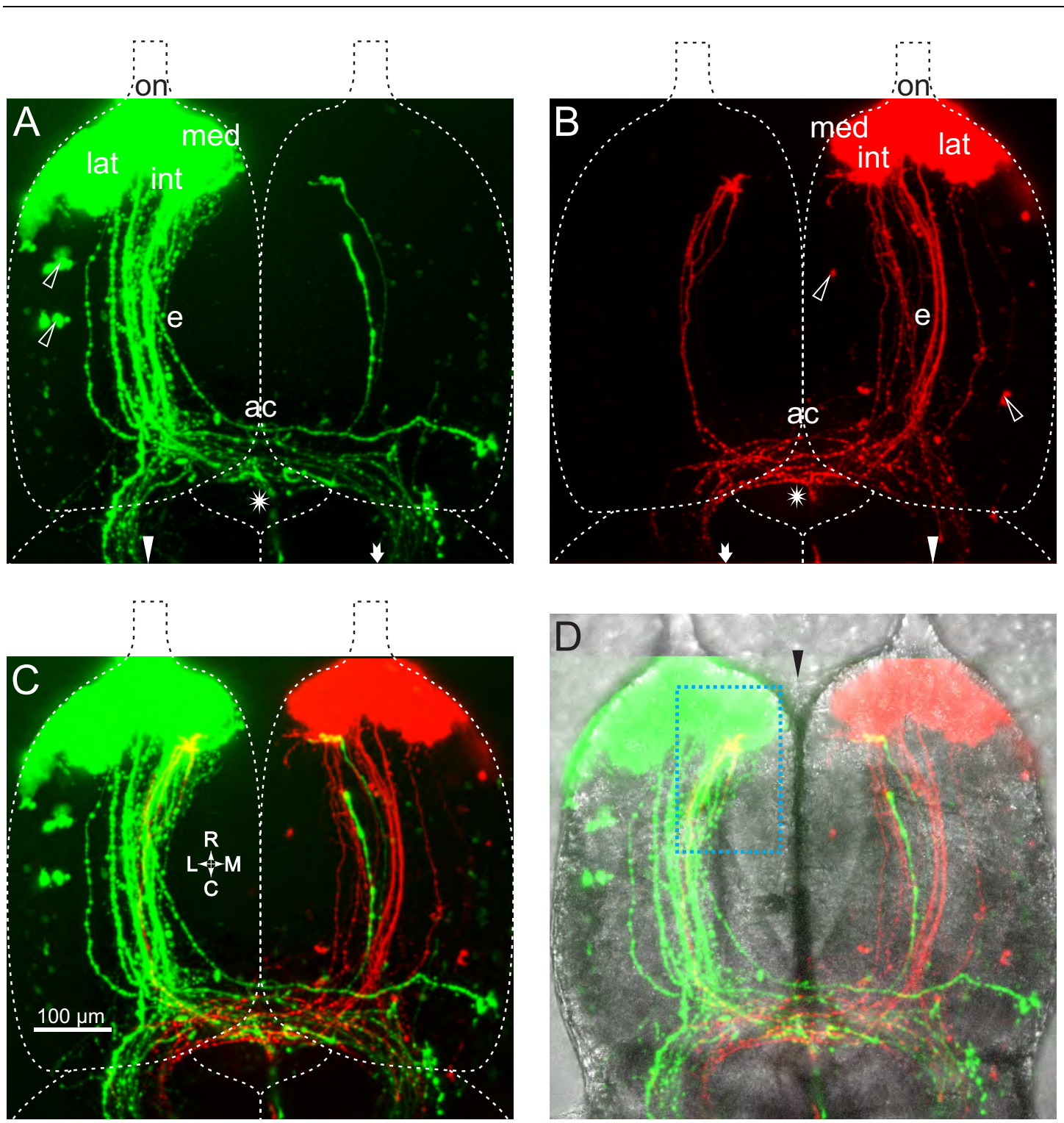

Figure 3.1: Overview of bilateral projections of axons of olfactory sensory neurons at stage 47

Maximum intensity projection (MIP) along the dorsoventral axis of a $128 \mu \mathrm{m}$ thick imaged volume (A to C) and a pseudo bright-field image (D) within the ventral OB. The left nasal cavity was electroporated with Alexa Fluor $48810 \mathrm{kDa}$ dextran. The signal from the Alexa Fluor 488 fluorophore is presented in the green channel. The right nasal cavity was electroporated with Alexa Fluor $56810 \mathrm{kDa}$ dextran. The signal from the Alexa Fluor 568 fluorophore is presented in the red channel.

A and B: Olfactory sensory neuron axons emerging from the left OE (A) or from the right $\mathrm{OE}(\mathrm{B})$ enter the $\mathrm{OB}$ at their respective ipsilateral side through the olfactory nerve and fan out to form the glomerular clusters. Several fibers bypass the glomerular layer and leave the OB caudally along the extrabulbar olfactory pathway towards the anterior 
The olfactory sensory neuron axons that follow the extrabulbar olfactory pathway to the contralateral hemisphere and proceed rostrally in the contralateral telencephalon do not belong to the EBOF, since these fibers terminate in a glomerular structure within the contralateral OB. A detailed image of this structure formed by the OSN axons that innervate the intermediate glomerular cluster within the contralateral $\mathrm{OB}$ is presented in Figure 3.2.

The electroporation tracing experiments with different dyes for the two nasal cavities of stage 47 tadpoles were performed in four animals. In all eight OBs of these animals the EBOF and the glomerular clusters formed by ipsilateral OSN axons could be identified. Olfactory sensory neuron axons that emerged from the contralateral $\mathrm{OE}$ and innervated the OB through the anterior commissure could be found in six of eight (75\%) cases. The lack of a contralateral staining within two OBs might be due to an incomplete tracing of OSNs by electroporation.

The novel innervation pathway consists of OSN axons running alongside the EBOF through the ipsilateral telencephalon towards and across the anterior commissure into the contralateral hemisphere. In the contralateral hemisphere the EBOF turn caudally

\section{Figure 3.1 continued:}

commissure. At the caudal end of the telencephalon these fibers divide into ipsilaterally projecting EBOF and contralaterally projecting fibers. The ipsilaterally projecting EBOF continue caudally, enter the ipsilateral diencephalon and project further caudally towards ipsilateral termination areas (marked by closed arrowheads). The contralaterally projecting fibers proceed through the anterior commissure towards the contralateral hemisphere. In the contralateral hemisphere single OSN axons turn rostrally and proceed towards the contralateral OB while residual EBOF turn caudally towards their contralateral termination areas (marked by arrows). Single EBOF leave the anterior commissure at the midline in caudal direction (marked by an asterisk). Examples of possible microglia are marked by open arrowheads.

C: The overlay of both channels shows that some OSN axons that enter the contralateral hemisphere through the anterior commisure follow the extrabulbar olfactory pathway in rostral direction. These fibers reach the contralateral $\mathrm{OB}$ and form a glomerular structure in the region of the intermediate glomerular cluster.

D: Overlay of both channels with a pseudo bright-field image of the same preparation. The closed arrowhead in $\mathrm{D}$ marks the anatomical separation between both OBs (midline). The dashed rectangle in D outlines the area presented in Figure 3.2.

Abbreviations: ac, anterior commissure; e, extrabulbar olfactory pathway; on, olfactory nerve; med, medial cluster; int, intermediate cluster; lat, lateral cluster; C, caudal; L, lateral; M, medial; R, rostral. 

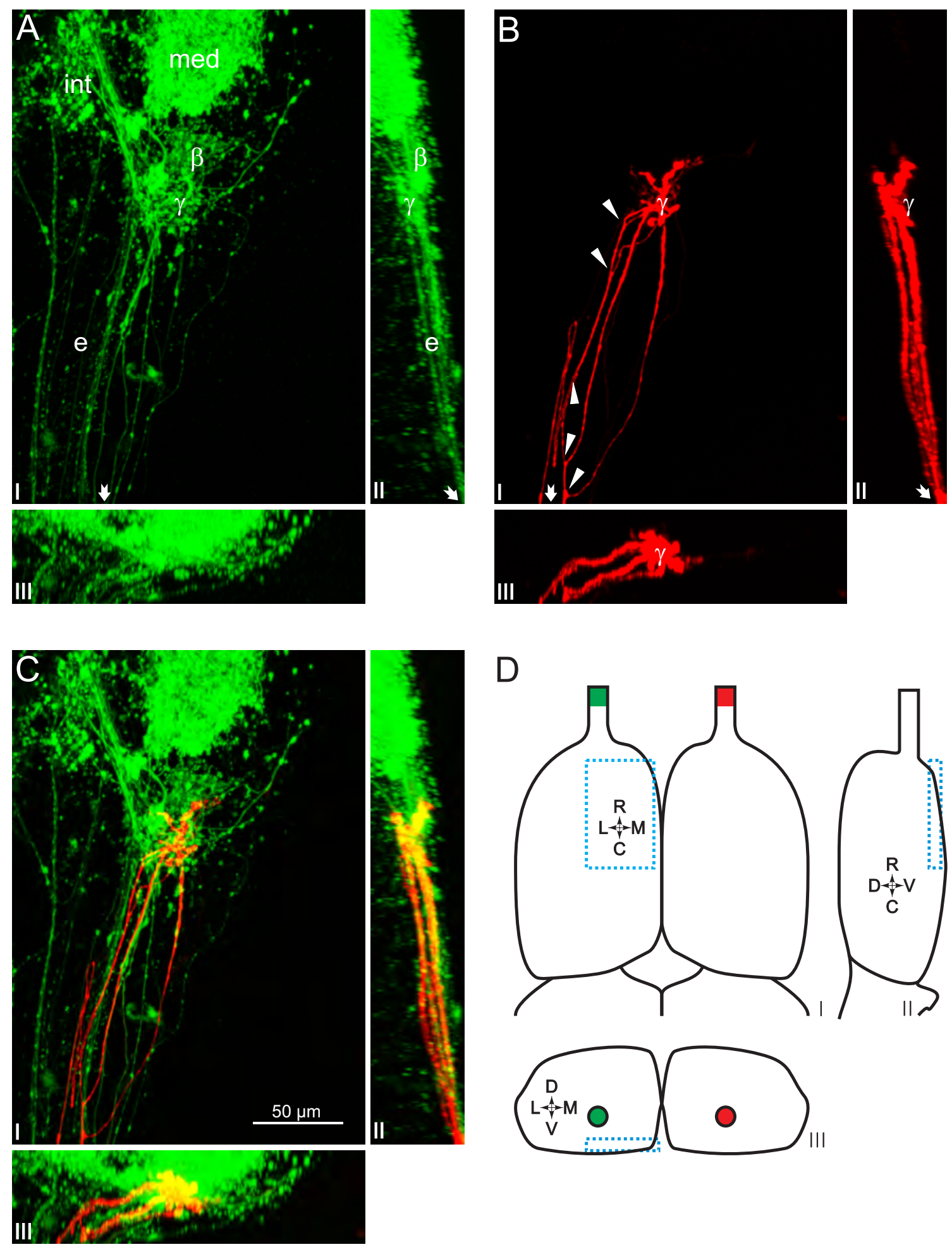

Figure 3.2: Glomerular tuft structure formed by axons of olfactory sensory neurons from the contralateral olfactory epithelium at stage 47

High resolution scan of the ventral OB (boxed area in Figure $3.1 \mathrm{D}$ ). The imaged volume is presented in separate MIPs for all three axes. Images marked with 'I' show the dorsoventral view where the imaged volume is projected along the dorsoventral axis. Images marked with 'II' show the lateral view where the imaged volume is projected along the mediolateral axis. Images marked with 'III' show the frontal view where the imaged volume is projected along the rostrocaudal axis. 
towards the diencephalon while the OSN axons of the novel innervation pathway turn rostrally towards the contralateral $\mathrm{OB}$. This new innervation pathway must be distinguished from previously reported OSN axons that enter the contralateral OB through the fusion of both OBs after stage 50 (Byrd and Burd, 1991; Gaudin and Gascuel, 2005; Nezlin and Schild, 2005). Since the OSN axons of this novel innervation pathway reach the contralateral $\mathrm{OB}$ by a deviation through the anterior commissure they are further referred to as 'deviating' OSN axons.

A detailed investigation of the innervation pattern by deviating OSN axons was performed on two OBs in stage 47 tadpoles (Figure 3.2). In both cases Gl $\gamma$ was the exclusive target for the deviating OSN axons.

The next step of this study was to investigate whether this contralateral innervation pathway changes in stage 52 tadpoles, after the dorsal parts of the OBs fuse along the midline and establish a direct route for OSN axons to the contralateral OB.

\section{Figure 3.2 continued:}

A: Olfactory sensory neuron axons emerging from the left, ipsilateral OE are presented in the green channel. Gl $\beta$ and Gl $\gamma$ lie completely within the imaged volume and appear partly fused along the rostrocaudal and dorsoventral axis. Olfactory sensory neuron axons of the extrabulbar pathway leave the OB in caudal direction (arrows).

B: Olfactory sensory neuron axons from the right, contralateral OE are presented in the red channel. These contralateral fibers enter the imaged volume at the ventrocaudal edge (arrows) and run rostrally, alongside with ipsilateral EBOF proceeding in the opposite direction. These fibers divide several times (arrowheads) until they reach the area of Gly and form glomerular tufts. The bifurcation pattern of the contralateral fibers resemble the multiple bifurcation of a single OSN axon in proximity of its target glomerula as described by Nezlin and Schild (2005). This interpretation of the apparent bifurcation of the contralateral olfactory fibers could not be verified as the imaging resolution is not sufficiently high to distinguish between single OSN axons and bundles including several OSN axons.

C: Overlay of both channels. The contralateral fibers run alongside the ipsilateral EBOF at the ventral rim of the telencephalon and approach Gl $\gamma$. The ipsilateral and contralateral fibers form Gl $\gamma$ by intermingling their glomerular tufts within the same area.

D: Location of the imaged volume within the telencephalon is schematically visualised by a dotted rectangle within each schematic view. The colour representing OSN axons emerging from respective $\mathrm{OE}$ is indicated by squares in $\mathrm{D}_{\text {I }}$ and circles in $\mathrm{D}_{\text {III. }}$.

Abbreviations: $\beta, \mathrm{Gl} \beta ; \gamma, \mathrm{Gl} \gamma$; e, extrabulbar olfactory fibers; med, medial cluster; int, intermediate cluster; $\mathrm{C}$, caudal; D, dorsal; L, lateral; M, medial; R, rostral; V, ventral. 


\subsubsection{Two different contralateral innervation pathways for the axons of olfactory sensory neurons at stage 52}

The tracing experiments with two different dyes for the two nasal cavities were repeated with stage 52 tadpoles. At this stage both OBs are fused in the dorsal part along the midline and the contralateral innervation within the PF9 is established (Gaudin and Gascuel, 2005). The aim of these experiments was to investigate if deviating OSN axons also exist in stage 52 tadpoles and if these deviating OSN axons can be morphologically differentiated from OSN axons that emerge from the contralateral OE and enter the OB directly through the fusion zone of PF9.

The contralateral innervation by OSN axons was analysed in eight OBs from four stage 52 tadpoles. The contralateral innervation of the dorsomedial cluster was detected in all eight OBs. In seven of the eight OBs deviating OSN axons were detected. In five cases the deviating OSN axons innervating exclusively Gl $\gamma$ were clearly separated from contralateral fibers innervating the dorsomedial cluster (Figure 3.3). In two cases the deviating OSN axons innervated not only Gl $\gamma$ : single fibers of deviating OSN axons also proceeded rostrally towards the intermediate and medial clusters. In these two cases a clear anatomical separation between the deviating OSN axons and OSN axons emerging from the contralateral $\mathrm{OE}$ and entering the $\mathrm{OB}$ directly through the dorsomedial fusion zone was not unambiguously possible.

In three OBs with exceptionally strong staining of the deviating OSN axons, these axons were followed towards the anterior commissure (Figure 3.4). This backtracking of deviating OSN axons demonstrated in all three specimens that deviating OSN axons take the same route at stage 52 as at stage 47. The deviating OSN axons run alongside

\section{Figure 3.3: Overview of projections of olfactory sensory neurons within the olfactory bulb at stage 52}

The imaged volume is presented in separate MIP for all three axes (I: dorsomedial; II: mediolateral; III: rostrocaudal).

A: Olfactory sensory neuron axons emerging from the left, ipsilateral OE are presented in the green channel. These fibers constitute the lateral, intermediate, dorsomedial and ventromedial clusters (separated by dashed lines). Within the intermediate cluster G1 $\beta$, Gl $\gamma$ and IS $\delta$ are distinguishable. Several EBOF leave the OB along the ventral rim of the $\mathrm{OB}$ in caudal direction (marked with arrowheads in $\mathrm{A}_{\mathrm{I}}$ and $\mathrm{A}_{\mathrm{II}}$ ).

B: Olfactory sensory neuron axons emerging from the right, contralateral OE are presented in the red channel. These contralateral OSN axons innervate the OB in two distinct areas: the dorsomedial cluster and Gl $\gamma$. The predominant part of the contralateral 

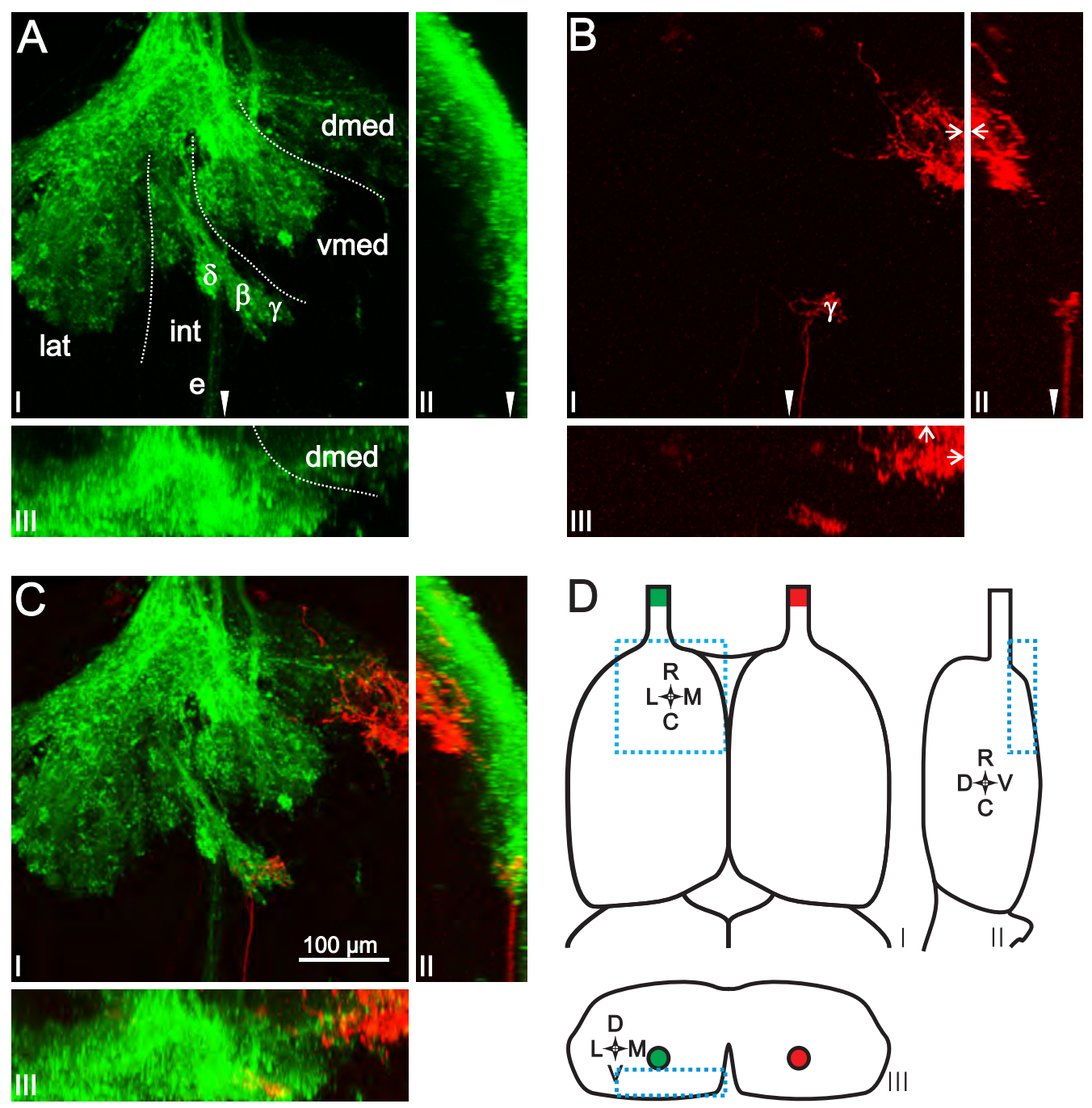

Figure 3.3 continued:

OSN axons enter the OB through the dorsally fused midline between both OBs (arrows) and terminate in the dorsomedial cluster (as reported by Gaudin and Gascuel, 2005). A small fraction of the contralateral OSN axons approach the OB from the caudal direction (arrowheads) and innervate Gl $\gamma$. This contralateral innervation of Gl $\gamma$ corresponds to the innervation by the deviating OSN axons in stage 47 tadpoles. There is no connection of the deviating OSN axons innervating Gl $\gamma$ and the residual contralateral OSN axons innervating the dorsomedial cluster in this specimen.

C: Overlay of both channels.

D: A schematic overview of the orientation of the $\mathrm{OB}$ in the orthogonal projections and the approximate position of the scanned volume within the OB (dotted rectangle). The colour representing OSN axons emerging from respective OE is indicated by squares in $\mathrm{D}_{\mathrm{I}}$ and circles in $\mathrm{D}_{\mathrm{III}}$.

Abbreviations: $\beta, \mathrm{Gl} \beta ; \gamma, \mathrm{Gl} \gamma ; \delta$, IS $\delta$; e, extrabulbar olfactory fibers; dmed, dorsomedial cluster; vmed, ventromedial cluster; int, intermediate cluster; lat, lateral cluster; $\mathrm{C}$, caudal; D, dorsal; L, lateral; M, medial; R, rostral; V, ventral. 
EBOF on the ventral border of the telencephalon (Figure $3.3 \mathrm{C}_{\mathrm{I}}$ and $\mathrm{C}_{\mathrm{II}}$ ) towards the anterior commissure. At the anterior commissure the deviating fibers intermingled with EBOF from the contralateral OE that progressed caudally towards the diencephalon.
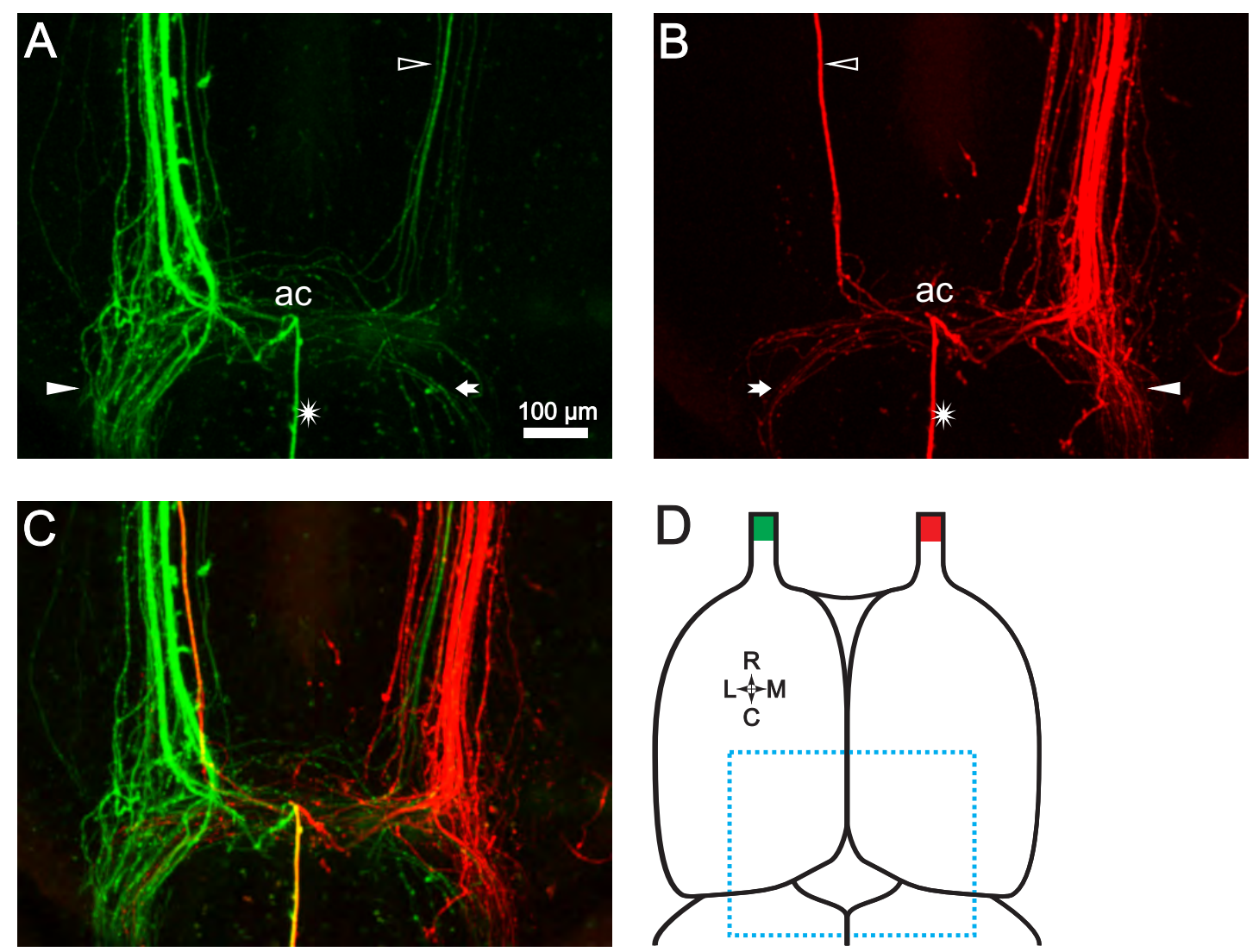

Figure 3.4: Projection of deviating axons of olfactory sensory neurons via the anterior commissure at stage 52

Maximum intensity projection (MIP) along the dorsoventral axis of a $145 \mu \mathrm{m}$ thick imaged volume at the anterior commissure. The left nasal cavity was electroporated with Alexa Fluor $48810 \mathrm{kDa}$ dextran; its fluorescence is presented in the green channel (A). The right nasal cavity was electroporated with Alexa Fluor $56810 \mathrm{kDa}$ dextran; its fluorescence is presented in the red channel (B). Both signals are overlaid in $\mathbf{C}$. The location of the imaged area is schematically presented in $\mathbf{D}$. The colour representing OSN axons emerging from the respective $\mathrm{OE}$ is indicated by squares in $\mathrm{D}_{\mathrm{I}}$ and circles in $\mathrm{D}_{\text {III. }}$ The olfactory sensory neuron axons which bypass the OB approach the anterior commissure and split up into ipsilaterally projecting EBOF (closed arrowheads) or contralaterally projecting axons. The contralaterally projecting axons proceed through the anterior commissure towards the contralateral hemisphere. In the contralateral hemisphere, deviated OSN axons turn rostrally and proceed towards the contralateral OB (open arrowheads) while contralaterally projecting EBOF (arrows) turn caudally towards their termination areas. Single EBOF leave the anterior commissure at the midline in caudal direction (asterisk).

Abbreviations: ac, anterior commissure; C, caudal; L, lateral; M, medial; R, rostral. 
These tracing results indicate the existence of two distinct OB innervation pathways by OSN axons emerging from the contralateral OE in stage 52 tadpoles. The first pathway consists of deviating OSN axons. The second pathway consists of OSN axons that enter the contralateral OB through the dorsomedial fusion of the OBs after stage 50.

The deviating OSN axons entered the OB caudally and innervated Gl $\gamma$ at stage 52 in a similar way as at stage 47 . In two cases the innervation by deviating OSN axons was not restricted to Gl $\gamma$ but appeared to extend into adjacent glomerular structures. This divergent innervation can be explained with misrouting of single OSN axons. Another possible explanation is the existence of further innervation areas for deviating OSN axons. To rule out this possibility, the innervation pattern of deviating OSN axons was studied in detail.

\subsubsection{Mapping of deviating axons of olfactory sensory neurons at stage 52}

The experiments described so far unambiguously showed the existence of deviating OSN axons that innervate the contralateral OB through the anterior commissure. The two cases with some deviating OSN axons that bypassed Gl $\gamma$ and strayed rostrally towards the intermediate and medial clusters made it necessary to perform additional experiments. Four additional stage 52 tadpoles were electroporated with two different dyes for the two nasal cavities and the innervation pattern of deviating OSN axons was studied in detail.

Figure 3.5 shows an example of the typical innervation pattern with all deviating axons terminating in glomerular tufts in Gl $\gamma$. The results of the complete analysis are presented in Table 1. 

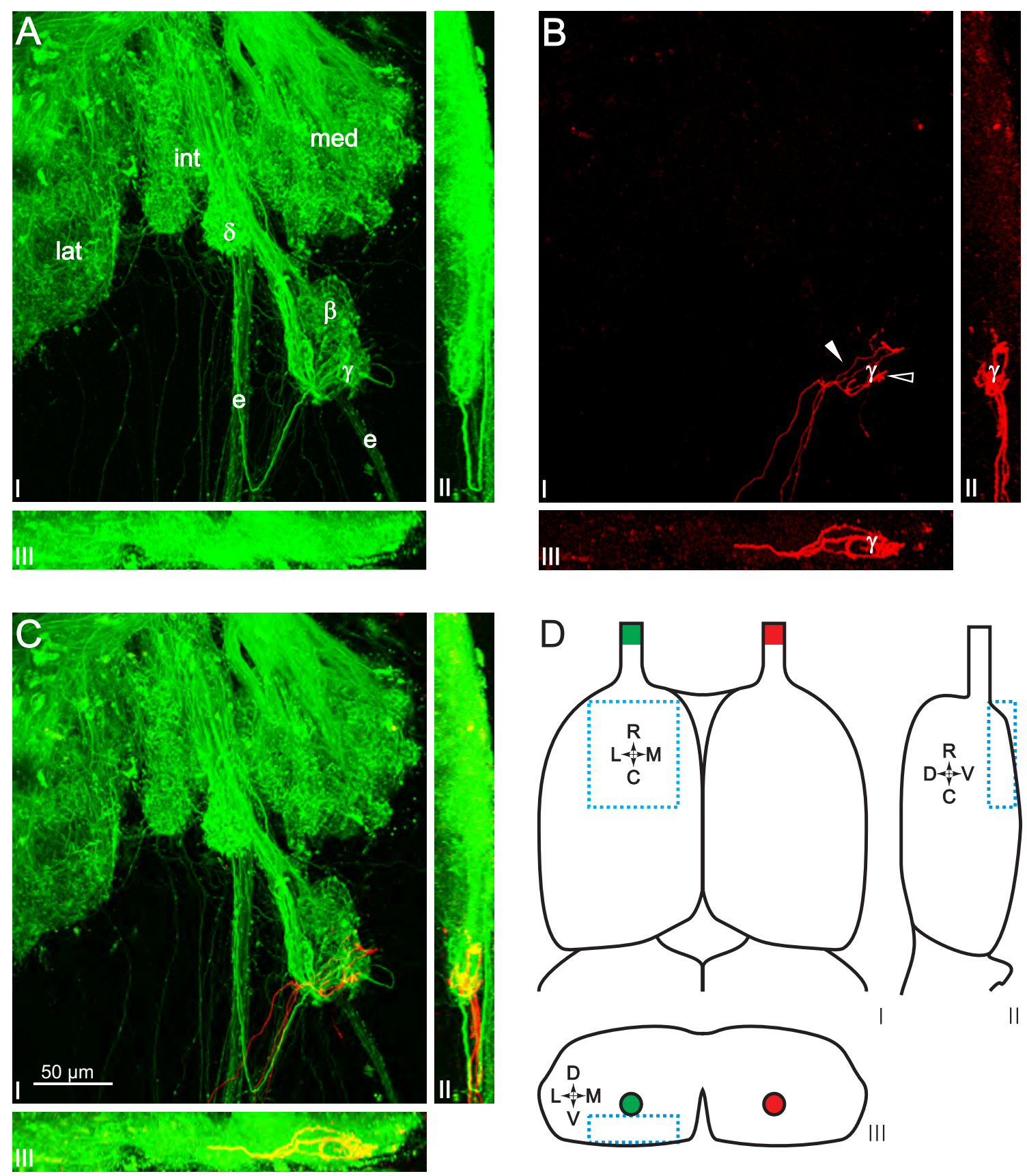

Figure 3.5: Specific innervation of Gly by deviating axons of olfactory sensory neurons at stage 52

The imaged volume is presented in orthogonal MIP for all three axes (I: dorsomedial; II: mediolateral; III: rostrocaudal).

A: Olfactory sensory neuron axons emerging from the left, ipsilateral OE constitute the glomerular clusters and EBOF. Individial glomeruli G1 $\beta$ and Gl $\gamma$ of the intermediate cluster appear fused along the rostrocaudal axis. These glomeruli lie dorsally to EBOF ( $\mathrm{A}_{\mathrm{II}}$. The extrabulbar olfactory fibers leave the OB and run caudally along the ventral rim of the telencephalon. 


\begin{tabular}{|c|c|c|c|}
\hline \multirow[b]{2}{*}{ Glomerular structure } & \multirow{2}{*}{$\begin{array}{l}\text { Number of } \\
\text { innervations }\end{array}$} & \multicolumn{2}{|c|}{ Innervation by } \\
\hline & & $\begin{array}{l}\text { filamentary } \\
\text { fibers only }\end{array}$ & $\begin{array}{c}\text { glomerular } \\
\text { tufts }\end{array}$ \\
\hline Gly & 14 & 0 & 14 \\
\hline G1 $\beta$ & 3 & 1 & 2 \\
\hline IS $\delta$ & 3 & 2 & 1 \\
\hline residual intermediate cluster & 3 & 2 & 1 \\
\hline medial cluster & 1 & 1 & - \\
\hline lateral cluster & 1 & 1 & - \\
\hline
\end{tabular}

Table 1: Innervation of different glomerular structures by deviating axons of olfactory sensory neurons at stage 52

Innervation pattern of deviating fibers from 16 OBs. The innervations were classified into two categories: innervation by filamentary fibers only (closed arrowhead in Figure $3.5 \mathrm{~B}$ ) or fibers that formed glomerular tufts (open arrowhead in Figure $3.5 \mathrm{~B}$ ). A quantification of the degree of innervation of individual glomeruli or cluster by deviating OSN axons was not performed: an individual glomerulus or cluster was counted as innervated when at least one deviating OSN axon entered this structure.

The deviating OSN axons were found in 14 out of 16 analysed OBs (88\%). In all 14 cases Gl $\gamma$ was innervated by deviating OSN axons with distinct glomerular tufts. Innervation of glomeruli other than Gl $\gamma$ was found only in six out of 16 specimens (37.5\%). The number of the deviating OSN axons outside of Gl $\gamma$ appeared to decrease with distance from Gl $\gamma$. The adjacent Gl $\beta$ was innervated in three specimens, in two cases with glomerular tufts. Two more cases of glomerular tufts detected outside of Gl $\gamma$

Figure 3.5 continued:

B: Deviating OSN axons emerging from the contralateral OE approach the OB from caudal direction and terminate exclusively in Gl $\gamma$. An example of deviating OSN axons that were classified as filamentary fibers is marked with a closed arrowhead. An example of presumed axon terminals is marked with an open arrowhead.

C: Overlay of both channels.

D: Schematic overview of the orientation of the OB in the orthogonal MIP and the approximate position of the scanned volume within the $\mathrm{OB}$ (dotted rectangle). The colour representing OSN axons emerging from the respective OE is indicated by squares in $\mathrm{D}_{\mathrm{I}}$ and circles in $\mathrm{D}_{\mathrm{III}}$.

Abbreviations: $\beta, \mathrm{Gl} \beta ; \gamma, \mathrm{Gl} \gamma ; \delta$, IS $\delta$; e, extrabulbar olfactory fibers; med, medial cluster; int, intermediate cluster; lat, lateral cluster; C, caudal; D, dorsal; L, lateral; M, medial; R, rostral; V, ventral. 
were restricted to the intermediate cluster, too. The medial and lateral clusters which lie remote from Gl $\gamma$ were innervated only in one specimen each. The deviating OSN axons appeared to run straight through these clusters without further bifurcation and glomerular tufts. These results indicate Gl $\gamma$ as the presumable target for deviating OSN axons.

\subsubsection{Characterisation of axons of olfactory sensory neurons invading the contralateral olfactory bulb across the fusion zone of PF9}

Additionally to the detailed study of deviating OSN axons, the new electroporation tracing technique allowed also a detailed investigation of the direct innervation of the contralateral OB by OSN axons through the fusion zone of PF9. The direct contralateral innervation by OSN axons was mentioned by Nezlin and Schild (2005) and by Gaudin and Gascuel (2005) for Xenopus laevis tadpoles after stage 50. However, these studies do not provide details about number and morphology of the direct contralateral innervation. In this study a detailed information about the amount and projections of direct contralateral innervating OSN axons within the OB are presented.

The experimental procedure to investigate the dorsal OB had to be slightly adjusted because the dorsal $\mathrm{OB}$ is not easily accessible with the explanted nose-olfactory bulb preparation used for the previous experiments. To perform detailed scans of the dorsal $\mathrm{OB}$, the tissue was fixed and sliced using a vibratome. Preliminary experiments showed that the fixation process substantially decreased the signal intensity from fibers traced with Alexa Fluor 488 and Alexa Fluor $56810 \mathrm{kDa}$ dextran. A sufficient staining of the OSN axons emerging from the contralateral $\mathrm{OE}$ was achieved by electroporation of biocytin into the contralateral nasal cavity and a subsequent visualisation by avidin coupled Alexa Fluor 488 (as described in the methods section 2.4.4). The ipsilateral nasal cavity was electroporated with Alexa Fluor $56810 \mathrm{kDa}$ dextran. The signal of Alexa Fluor 568 was sufficient to identify the glomerular clusters in the ipsilateral side while the signal of the Alexa Fluor 488 fluorophore was strong enough to follow even single fibers invading the $\mathrm{OB}$ from the contralateral side as presented in Figure 3.6. 
These tracing experiments were performed with three animals. All specimens showed a pronounced intermingling of OSN axons emerging from both OE at the fusion zone of PF9. In all cases the number of OSN axons from the contralateral side decreased with increasing distance from this fusion zone. Single fibers of contralateral OSN axons could be followed throughout the whole extent of the dorsomedial cluster and formed glomerular tufts up to $300 \mu \mathrm{m}$ from the midline (Figure $3.6 \mathrm{G}$ to I). It was not possible to determine whether PF1 is innervated by contralateral OSN axons because no morphological boundary between PF9 and PF1 could be found. It was not possible to distinguish between these two projection fields. No innervation of the most ventral projection field of the medial cluster (PF2) was found. This is in line with the results described in the previous section 3.1.3.

\subsubsection{Summary: bilateral tracing experiments}

The tracing experiments performed with two different dyes for the two nasal cavities revealed a new pathway for OSN axons to innervate the contralateral OB after entering the contralateral hemisphere through the anterior commissure. These deviating OSN axons consistently innervated Gl $\gamma$. While the deviating OSN axons were detected already at stage 47 , the fusion of both OBs at stage 50 enables a second, direct pathway for contralateral OSN axons to innervate the medial cluster of the OB. The degree of innervation by this second, direct pathway decreased with the distance to the fusion zone.

Gl $\gamma$ is the presumed target for the deviating OSN axons at stage 52. The next step in understanding the function of this new contralateral innervation pathway was to analyse the physiological role of Gl $\gamma$ by exploring the responses of this structure to stimulation of the $\mathrm{OE}$. 

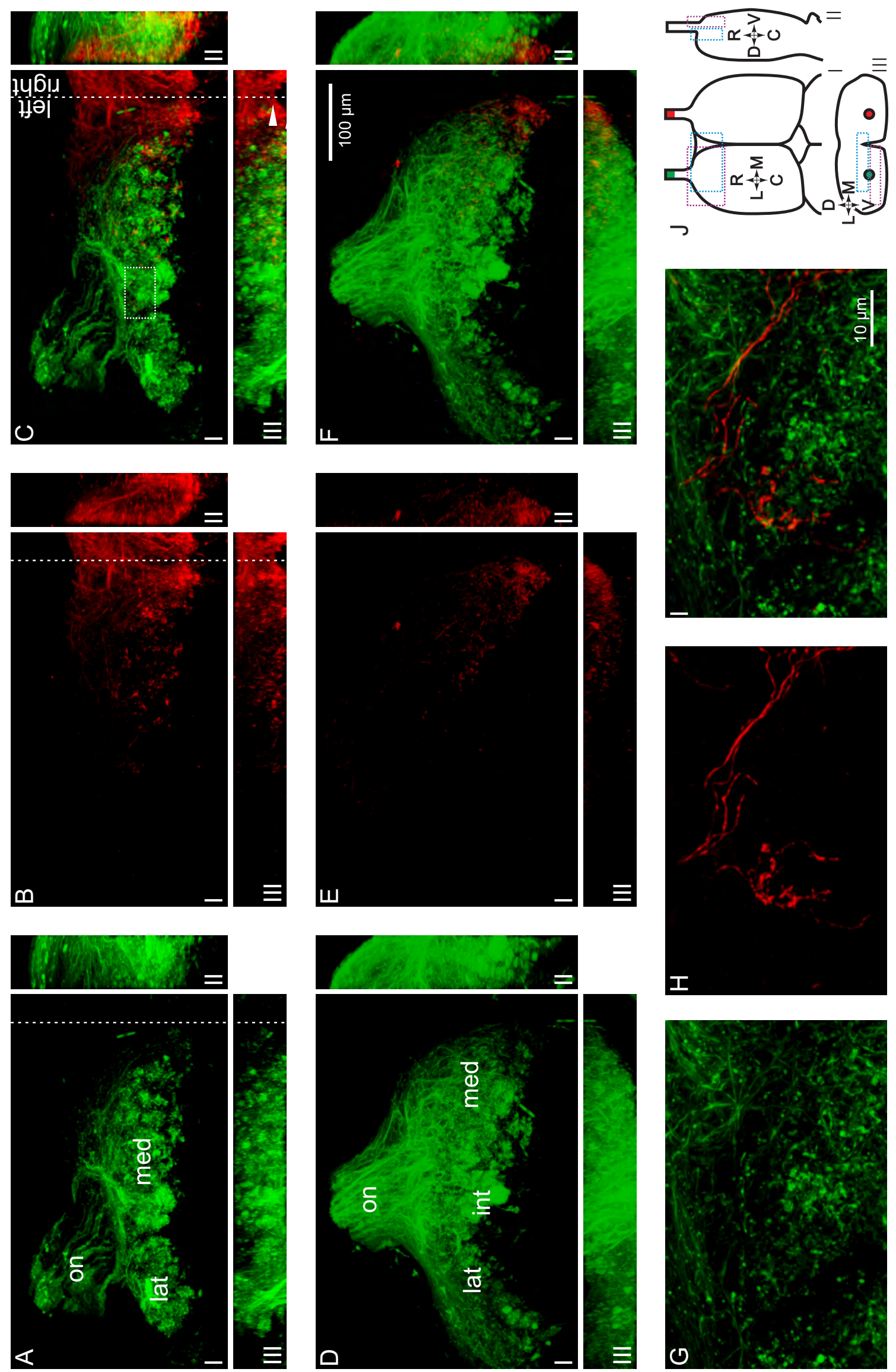

Figure 3.6: The direct contralateral innervation within the dorsomedial cluster 


\section{Figure 3.6: The direct contralateral innervation within the dorsomedial cluster}

The signal from OSN axons emerging from the ipsilateral OE is presented in the green channel. The signal from OSN axons emerging from the contralateral OE is presented in the red channel. Two imaged volumes are presented in separate MIP for all three axes (I: dorsoventral; II: mediolateral; III: rostrocaudal).

A to $\mathbf{C}$ : Vibratome slice containing the ventral rim of the PF9 fusion area. The midline separating the ipsilateral (left) $\mathrm{OB}$ and the contralateral (right) $\mathrm{OB}$ is marked with a dotted vertical line. The location of the imaged volume is schematically shown with blue dotted rectangles in J. Olfactory sensory neuron axons emerging from the ipsilateral $\mathrm{OE}$ enter the $\mathrm{OB}$ through the olfactory nerve and divide into the medial and lateral cluster. The intermediate cluster cannot be distinguished at this height. The medial cluster shows the most prominent staining at its temporal border and a decreasing staining intensity towards the midline. Ipsilateral fibers that cross the midline are barely visible because of the weak staining. Olfactory sensory neuron axons from the contralateral (right) $\mathrm{OE}$ enter through the right surface of the imaged volume. The amount of contralateral fibers decreases with distance to the midline. In the lateral cluster no innervation by contralateral OSN axons can be detected. In the overlay of frontal projections $\left(\mathrm{C}_{\mathrm{III}}\right)$ the ventral edge of the $\mathrm{OB}$ fusing zone is marked with an arrowhead. Dorsal to this edge contralateral fibers cross from the right to the left OB. Ventral to this edge, the OBs are anatomically separated.

D to F: Vibratome slice located ventrolaterally from the area presented in A to C (location schematically shown with purple dotted rectangles in J). The ipsilateral OSN axons enter the OB through the olfactory nerve and fan out to the medial, intermediate and lateral clusters $\left(\mathrm{D}_{\mathrm{I}}\right)$. The contralateral OSN axons enter the scanned volume through the dorsal surface $\left(\mathrm{E}_{\mathrm{II}}\right.$ and $\left.\mathrm{E}_{\mathrm{III}}\right)$. The amount of contralateral fibers decreases with increasing distance to the fusing zone of PF9 in lateral $\left(\mathrm{E}_{\mathrm{I}}\right)$ and ventral direction $\left(\mathrm{E}_{\mathrm{III}}\right)$. The intermediate and lateral cluster are not innervated by contralateral fibers.

G to I: Magnification of the small region marked in $\mathrm{C}$ is presented as a dorsoventral MIP. The contralateral fibers enter the field of view from the medial direction and form glomerular tuft structures with ipsilateral fibers.

J: Schematic location of both imaged volumes within the telencephalon (A to C blue, $\mathrm{D}$ to $\mathrm{F}$ purple) and their respective location to each other. The colour representing OSN axons emerging from the respective $\mathrm{OE}$ is indicated by squares in $\mathrm{J}_{\mathrm{I}}$ and circles in $\mathrm{J}_{\mathrm{III}}$.

Abbreviations: med, medial cluster; int, intermediate cluster; lat, lateral cluster; on, olfactory nerve; C, caudal; D, dorsal; L, lateral; M, medial; R, rostral; V, ventral. 


\subsection{Calcium imaging of axons of olfactory sensory neurons within the olfactory bulb}

The responses of OSN axons within the OB upon stimulation of the OE were explored on explanted nose-olfactory bulb preparations from stage 52 tadpoles. The olfactory sensory neurons were loaded with a calcium sensitive dye (fluo-4 $10 \mathrm{kDa}$ dextran) using electroporation. The preparation of the $\mathrm{OB}$ and nose was performed as described in the methods section. The explanted nose-olfactory bulb preparation was placed in the recording chamber and the outlet of the stimulation funnel was placed in front of the left OE.

\subsection{1 $\left[\mathrm{Ca}^{2+}\right]_{i}$ transients are not restricted to the presynaptic terminals of olfactory sensory neurons}

Calcium imaging experiments revealed an interesting observation concerning calcium signalling within OSN axons. A response to stimulation of the OE was not only detected within OSN axon terminals forming glomerular tufts (Figure $3.7 \mathrm{~B}$, open arrowhead) but also in the whole extent of the OSN axons including the sorting zone and the olfactory nerve (Figure 3.7 B, closed arrowhead). This finding is in contrast to previous calcium imaging studies in traced OSN axons of zebrafish (Friedrich and Korsching, 1997) and mouse (Wachowiak et al., 2004). The result has significant relevance for the interpretation of the following calcium imaging experiments. The solitary location of Gl $\beta$ and Gl (Figure 3.5 A) makes it possible to distinguish the presynaptic terminals from the bypassing axons projecting towards other glomeruli. Within densely packed glomerular clusters this separation is not possible. It can not be excluded that fibers innervating adjacent glomeruli could pass through the measured glomerulus and thus affect the measured response.

While this intriguing observation was accounted for in the analysis of the calcium imaging experiments, the identification of channels that are involved in the calcium influx during a spike train in the OSN axons of Xenopus laevis was beyond the scope of this thesis. 

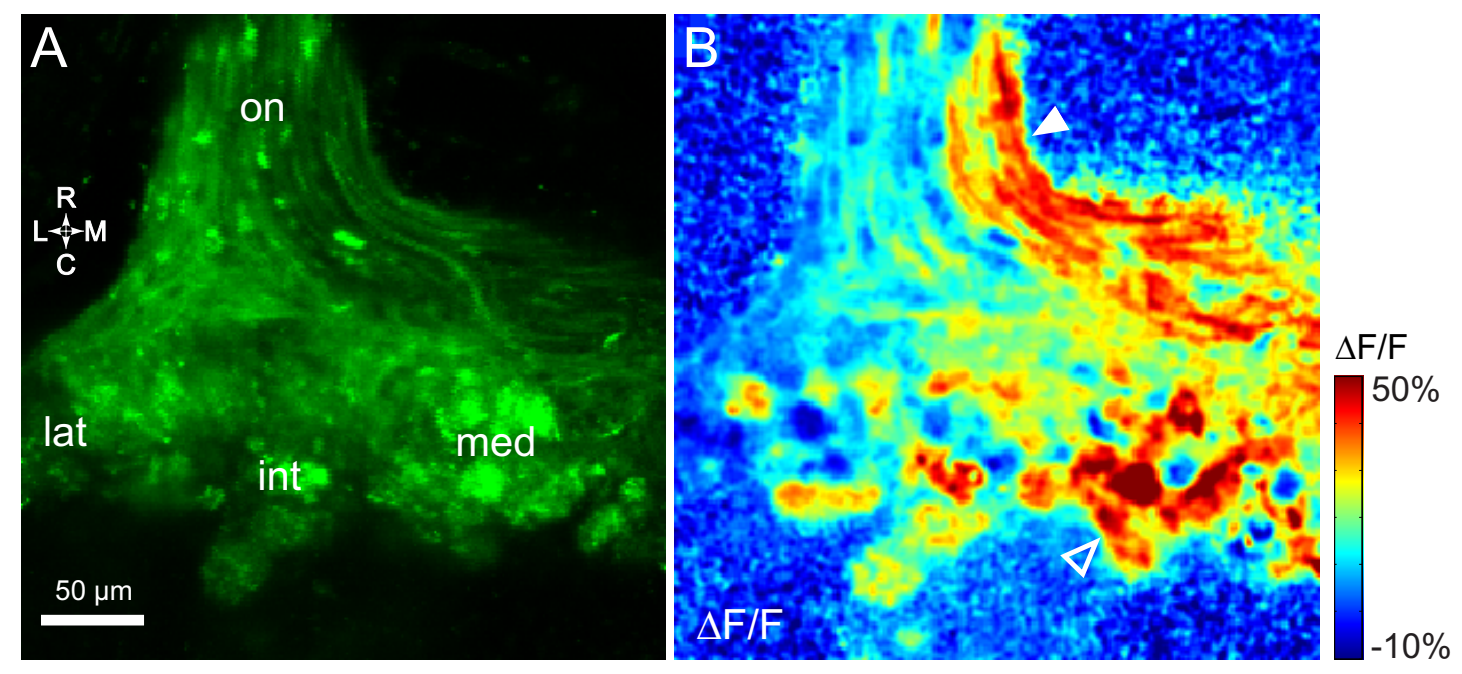

Figure 3.7: $\left[\mathrm{Ca}^{2+}\right]_{i}$ transients within the olfactory nerve as a response to odorant stimulation

A: Basal fluorescence of fluo-4 electroporated primary olfactory projection at the height of the olfactory nerve.

B: Peak response as $\Delta \mathrm{F} / \mathrm{F}$ map upon odorant stimulation with Altner alcohol mixture. Open arrowhead marks strongly responding glomeruli. Closed arrowhead marks $\left[\mathrm{Ca}^{2+}\right]_{\mathrm{i}}$ influx within the olfactory nerve.

Abbreviations: on, olfactory nerve; med, medial cluster; int, intermediate cluster; lat, lateral cluster; C, caudal; L, lateral; M, medial; R, rostral.

\subsubsection{Overview of the stimulus space of GI $\beta$ and Gl $\gamma$}

The calcium imaging experiments that were performed to find the adequate stimulus of Gl $\gamma$ additionally revealed the responses of G1 $\beta$ because of its adjacent location. This section gives an overview of the responses of both glomeruli to various stimuli. The specific results for the separate glomeruli are presented in detail in following sections.

The morphological identification of Gl $\beta$ and Gl $\gamma$ by the fluorescence signal of the fluo- 4 fluorophore is difficult because of the very low fluo-4 fluorescence signal from vital OSN axons with their low basal $\left[\mathrm{Ca}^{2+}\right]_{\mathrm{i}}$ (Figure $3.8 \mathrm{~A}$ ). To simplify the localisation of Gl $\gamma$ within the ventral OB, the experimental animals were additionally electroporated with Alexa Fluor $56810 \mathrm{kDa}$ dextran in the right nasal cavity. The deviating OSN axons emerging from the right OE presumably innervate specifically Gl $\gamma$ within the left OB as presented in the previous chapter 3.1 (Figure $3.8 \mathrm{~B}$ ). The optical slice was placed through both glomeruli (Figure $3.8 \mathrm{C}$ ). 

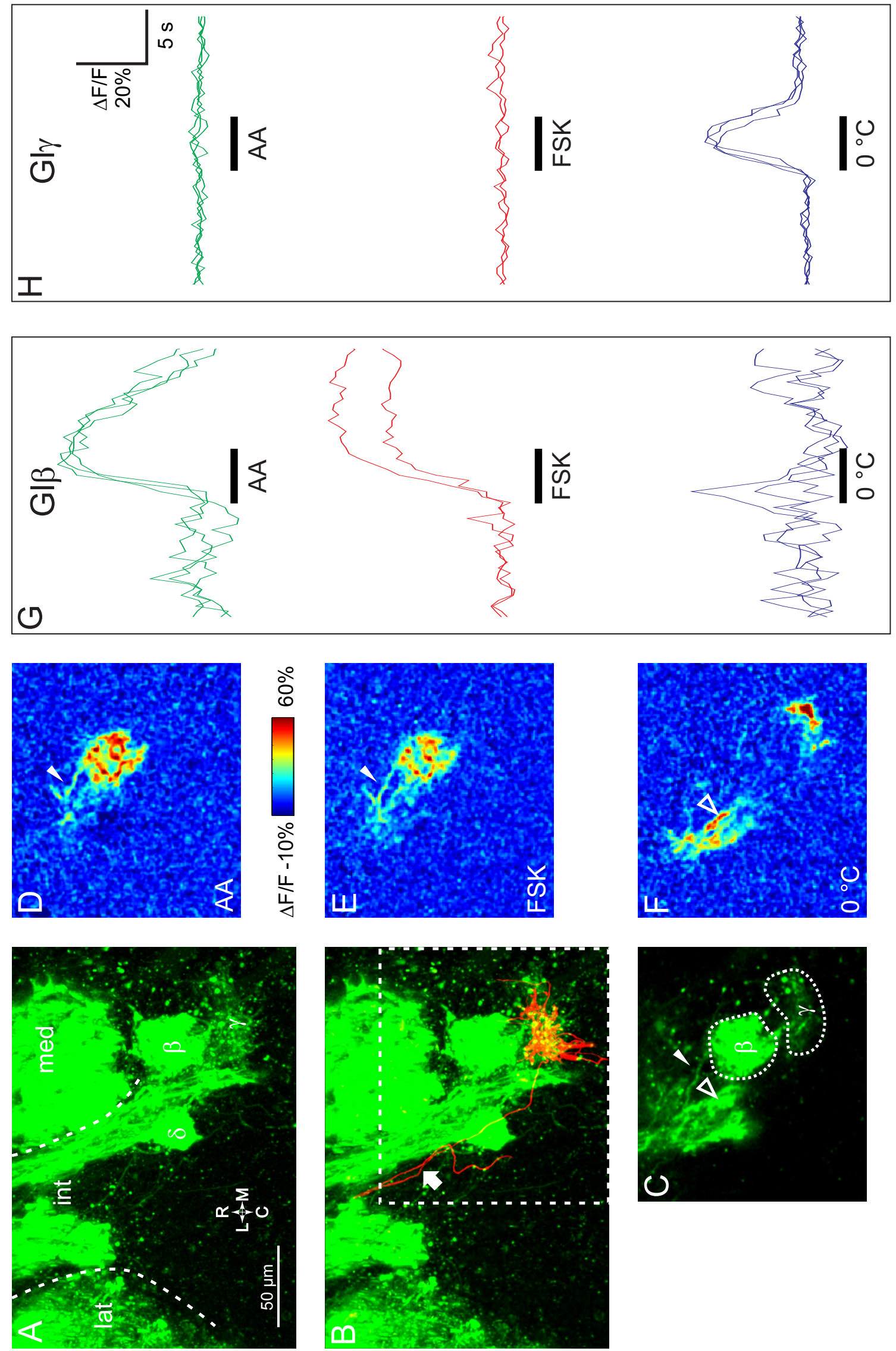

Figure 3.8: Overview of stimulus specificity in GI and Gly 
The transient $\left[\mathrm{Ca}^{2+}\right]_{i}$ response was not limited to the glomeruli as some concurrent response from OSN axons adjacent to these glomeruli was observed. As described in the previous section, this signal originated from OSN axons that presumably terminated within these glomeruli. Examples of these concurrent responses of OSN axons outside the glomeruli are marked in Figure $3.8 \mathrm{C}$ to $\mathrm{F}$.

To find the adequate stimuli for Gl $\gamma$ and Gl$\beta$, different odorants were tested. The results of these experiments are summarized in Table 2. Water soluble odorants that are known to stimulate OSNs of Xenopus laevis were applied in mixtures as described in the methods section. Forskolin was used as a pseudostimulus to stimulate all OSNs that respond to cAMP-transduced odors (Manzini and Schild, 2003b). Out of all used odorants G1 $\beta$ responded repeatedly to the amino acid mixture and to forskolin (Figure 3.8 G, Table 2). No reaction of Gly upon application of water soluble odorants could be detected.

\section{Figure 3.8: Overview of stimulus specificity in GI $\beta$ and Gl $\gamma$}

A and B: Morphological overview of the scanned region as MIP along the dorsoventral axis. The signal from fluo-4 $10 \mathrm{kDa}$ dextran dye which was electroporated in the ipsilateral OE is presented at basal fluorescence level in the green channel. The signal from Alexa Fluor $56810 \mathrm{kDa}$ dextran dye is presented in the red channel (only as overlay in B). A single deviating fiber which does not innervate Gl $\gamma$ is marked with an arrow.

C: Basal fluo-4 fluorescence signal of single optical slice through G1 $\beta$ and Gl $\gamma$ (boxed area in B) used for time series measurements .

D to $\mathbf{F}$ : Peak response $\Delta \mathrm{F} / \mathrm{F}$ maps during stimulation with amino acids (D), forskolin (E) and cold Ringer solution (F). Single fibers that reacted during the stimulation but lie outside of G1 $\beta$ or Gl $\gamma$ are marked with a closed arrowhead (G1 $\beta)$ or an open arrowhead (Gl $\gamma)$. The Gl $\gamma$ innervating axons lie partly outside of the imaged slice, see A for their continuous course.

$\mathbf{G}$ and $\mathbf{H}: \Delta \mathrm{F} / \mathrm{F}$ response traces from $\mathrm{Gl} \beta(\mathrm{G})$ and $\mathrm{Gl} \gamma(\mathrm{H})$ upon repeated stimulation with amino acids $(3 \mathrm{x})$, forskolin $(2 \mathrm{x})$ and cold Ringer solution (3x). Start and length of applications are marked with a black bar. ROIs used for calculating the time traces are outlined in $\mathrm{C}$.

Abbreviations: $\beta$, G1 $\beta ; \gamma$, Gl $\gamma ; \delta$, IS $\delta$; AA, amino acids; FSK, forskolin; med, medial cluster; int, intermediate cluster; lat, lateral cluster; C, caudal; L, lateral; M, medial; R, rostral. 
To find an adequate stimulus for Gl $\gamma$, several alternative stimulations of OSNs were tested. Amazingly, Gl $\gamma$ reproducibly responded to ice-cold Ringer solution that was pipetted into the application funnel (Figure $3.8 \mathrm{H}$ ). This response could be reproduced with repeated application of the cold stimulus in the same preparation as well as in four additional preparations. In all experiments control applications of Ringer solution at room temperature were performed to exclude the possibility of unspecific artefacts.

\begin{tabular}{lccc}
\hline Stimuli & Number of preparations & Response of Gl乃 & Response of Gl $\boldsymbol{y}$ \\
\hline forskolin & 12 & 12 & - \\
amino acids & 12 & 12 & - \\
amines & 7 & - & - \\
bile acids & 6 & - & - \\
nucleotides & 4 & - & - \\
altner alcohols & 8 & - & - \\
$\mathrm{CO}_{2}$ & 6 & - & - \\
ice-cold Ringer & 5 & - & 5 \\
flow disruption & 4 & 3 & - \\
\hline
\end{tabular}

Table 2: Response spectra of GI $\beta$ and Gl $\gamma$

Number of preparations with responses of Gl $\beta$ or Gl $\gamma$ to different stimuli. Single specimens were tested with varying stimulus configurations. The number of stimulated preparations is provided for each stimulus.

Occasionally, slight movements of the tissue were observed during temperature stimulation. To exclude activation of OSNs innervating Gl $\gamma$ due to movements, experiments with a mechanical stimulus were performed. This mechanical stimulus consisted of a complete interruption of the perfusion (see the methods section 2.5.3 for details). While strong responses to the mechanical stimulation were found in G1 $\beta$, no reaction of Gl $\gamma$ was detected (Figure 3.9). 

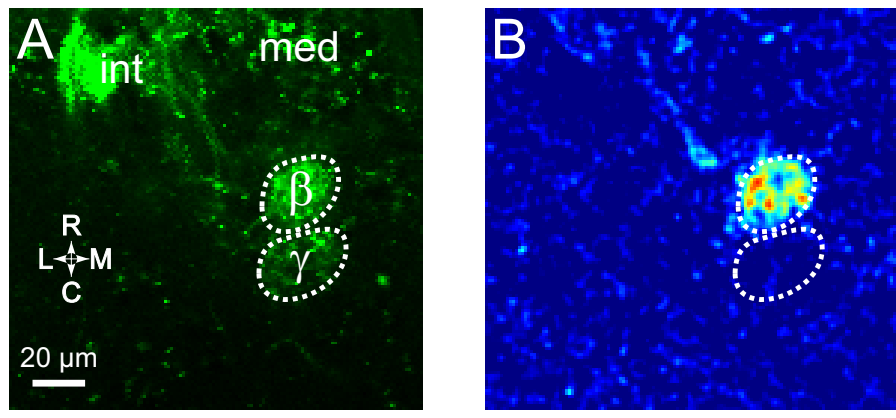

$\Delta F / F \quad-5 \% \square 50 \%$

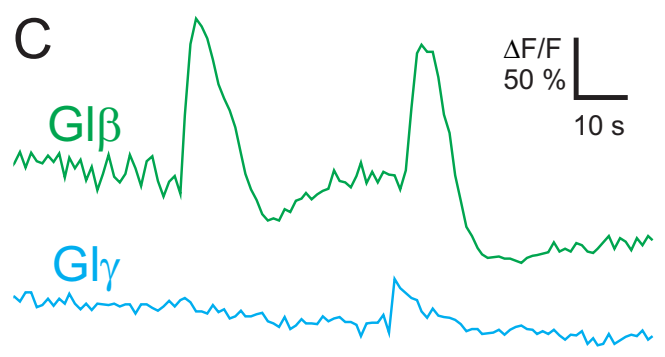

perfusion off

on

Figure 3.9: Response of GI $\beta$ to mechanical stimulation

A: Basal fluorescence of fluo-4 electroporated OSN axons in the ventral OB.

B: $\Delta \mathrm{F} / \mathrm{F}$ map of peak response to mechanical stimulation by interrupting of the Ringer perfusion.

C: $\Delta \mathrm{F} / \mathrm{F}$ time trace of G1 $\beta$ and Gl $\gamma$. G1 $\beta$ responds to the stop of perfusion as well as to the restart of the perfusion with $\left[\mathrm{Ca}^{2+}\right]$ i transients. Gl $\gamma$ shows only spontaneous activity that is uncorrelated to the mechanical stimulus. Regions that were used for the calculation of the time traces are marked in A and B by dotted lines.

Abbreviations: $\beta, \mathrm{Gl} \beta ; \gamma, \mathrm{Gl} \gamma$; med, medial cluster; int, intermediate cluster; $\mathrm{C}$, caudal; $\mathrm{L}$, lateral; M, medial; R, rostral.

\subsubsection{Spatial distribution of the GIß response to stimulation with amino acids and forskolin}

G1 $\beta$ shows calcium responses upon stimulation with both amino acids and forskolin. Previous studies of the olfactory system of Xenopus laevis tadpoles indicated an almost exclusive response to either of these two stimuli. From the population of OSNs sensitive to amino acids and/or forskolin, only $5.12 \%$ responded to both stimuli, while $47.7 \%$ responded exclusively to amino acids and $47.2 \%$ responded exclusively to forskolin (Manzini and Schild, 2003b). In calcium imaging experiments using OB slices stained by fluo-4/AM bath incubation no overlapping calcium response within the glomerular 
layer to stimulation with amino acids or forskolin was found (Manzini et al., 2007b). This major subdivision (amino acid sensitive OSN axons projecting into the lateral cluster and forskolin sensitive OSN axons projecting into the medial cluster) was also observed in calcium imaging experiments of OSN axons within the ventral $\mathrm{OB}$ in this thesis. Figure 3.10 shows peak response $\Delta \mathrm{F} / \mathrm{F}$ images after stimulation with amino acids (Figure $3.10 \mathrm{C}$ ) or forskolin (Figure $3.10 \mathrm{D}$ ). The responding regions can be roughly divided into the lateral cluster which responded to amino acids and the medial cluster which responded to forskolin. Several parts of the intermediate cluster responded either to amino acids or forskolin. Only G1 $\beta$ responded to both stimuli (Figure $3.10 \mathrm{C}$ and D, arrow).

G1 $\beta$ is the first described glomerulus with concurrent sensitivity to amino acids and forskolin. This concurrent sensitivity is caused either by OSN axons that are sensitive to both stimuli or by two distinct populations of OSN axons that are sensitive either to amino acids or forskolin but project into the same glomerulus. If Gl $\beta$ contains two distinct populations of axons that respond to mutually exclusive stimuli, this should be visible at high resolution as divergent spatial distribution of $\left[\mathrm{Ca}^{2+}\right]_{i}$ transients. To exclude this possibility of heterogeneous innervation, the spatial distribution of the calcium signal within G1 $\beta$ was analysed in detail. Responses of G1 $\beta$ to amino acids and forskolin were additionally scanned at high resolution in three specimens (Figure $3.10 \mathrm{E}$ to $\mathrm{H})$. These scans showed only small differences in the spatial distribution of the $\Delta \mathrm{F} / \mathrm{F}$ signal between applications of different stimuli as well as between repeated applications of the same stimulus. The responding regions to amino acids and forskolin were predominantly congruent in all three specimens.

The high resolution scans verified that OSN axons constituting G1 $\beta$ respond likewise to amino acids and forskolin. The response was detectable in the entire morphological structure of G1 $\beta$, indicating that G1 $\beta$ acts as a uniform, physiological unit.

\section{Figure 3.10: Response of GIß to stimulation with amino acids and forskolin}

A and B: Morphological overview of the imaged volume as MIP along the dorsoventral axis. The signal from fluo-4 $10 \mathrm{kDa}$ dextran, which was electroporated in the ipsilateral $\mathrm{OE}$, is presented at basal fluorescence in the green channel. The signal from Alexa Fluor $56810 \mathrm{kDa}$ dextran, which was electroporated in the contralateral $\mathrm{OE}$, is presented in the red channel (only as overlay in B). 

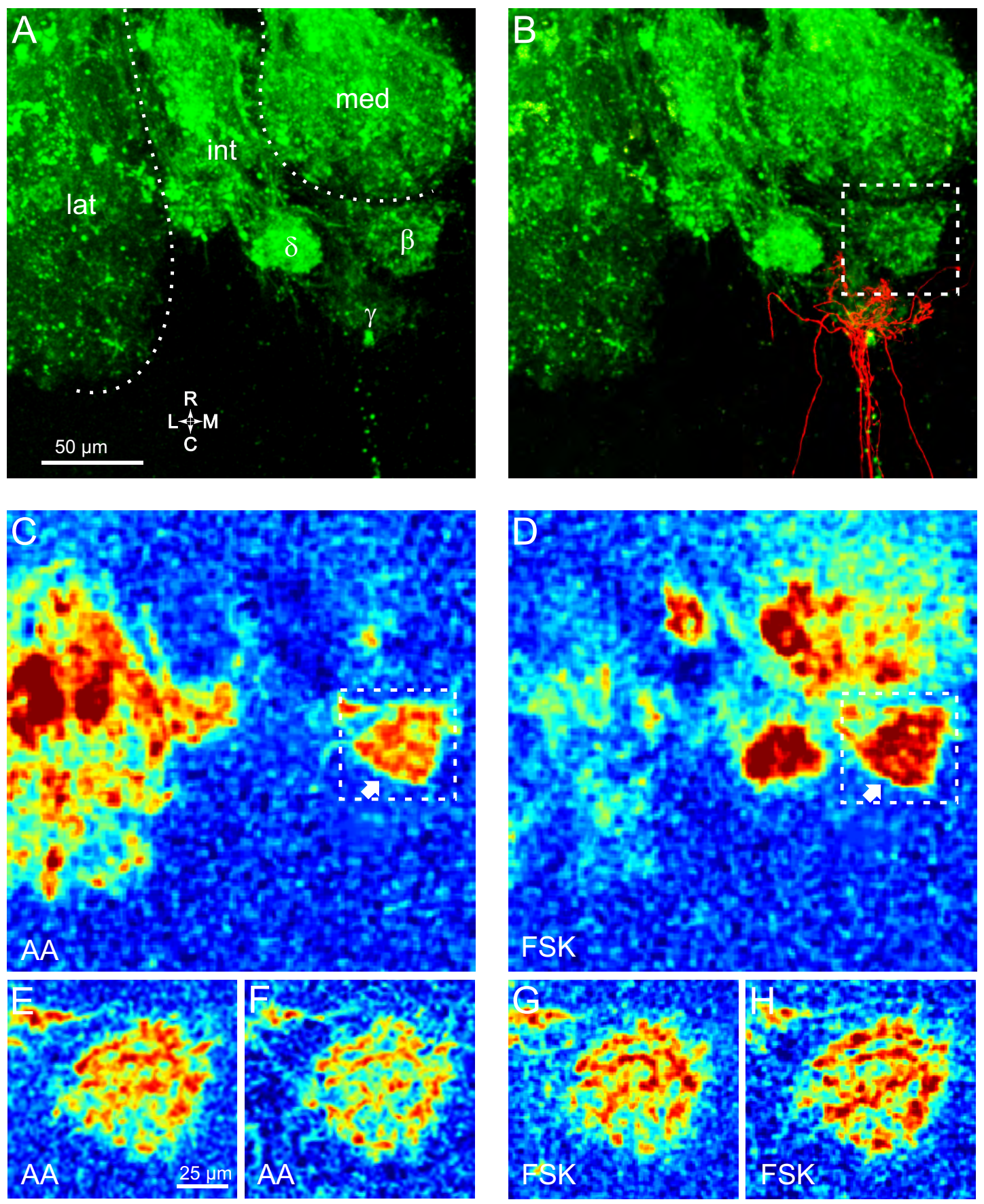

Figure 3.10 continued:

$\mathbf{C}$ to $\mathbf{H}$ : Spatial $\Delta F / F$ map at peak response upon stimulation with amino acid mix $(\mathrm{C}, \mathrm{E}$, F) or forskolin $(D, G, H)$. Gl $\beta$ is marked with an arrow in C and D. Response in Gl $\beta$ and lateral cluster to stimulation with amino acids in C. Response in Gl $\beta$ and medial cluster to stimulation with forskolin in D. Magnification of repeated odorant applications in $\mathrm{F}$ to $\mathrm{H}$ (boxed areas in $\mathrm{B}, \mathrm{C}$ and $\mathrm{D}$ ). The single stimulations were performed in the sequence E-G-F-H.

Abbreviations: $\beta$, G1 $\beta ; \gamma$, Gl $\gamma ; \delta$, IS $\delta$; AA, amino acids; FSK, forskolin; med, medial cluster; int, intermediate cluster; lat, lateral cluster; C, caudal; L, lateral; M, medial; R, rostral. 


\subsubsection{Temperature sensitivity of Gl $\gamma$}

\subsubsection{Gl $\gamma$ shows calcium increase in ipsilateral axons of olfactory sensory neurons upon cold temperature stimulation}

To study the temperature sensitivity of Gl $\gamma$ the explanted nose-olfactory bulb preparation was stimulated with graduated temperature stimuli. The results of these measurements within a single preparation are presented in Figure 3.11 A to F.

The olfactory epithelium was stimulated by application of Ringer solution attuned to the temperature $T_{R}$ into the perfusion funnel. The application caused a peak temperature difference $\Delta \mathrm{T}_{\mathrm{p}}$ to the temperature at the beginning of the experiment. The temperature difference measured at the $\mathrm{OE}$ upon temperature stimulation ranged from $\Delta \mathrm{T}_{\mathrm{p}}=-3.2 \pm 0.2{ }^{\circ} \mathrm{C} \quad\left(\mathrm{T}_{\mathrm{R}}=0{ }^{\circ} \mathrm{C}\right)$ to $\Delta \mathrm{T}_{\mathrm{p}}=+1.6 \pm 0.2{ }^{\circ} \mathrm{C} \quad\left(\mathrm{T}_{\mathrm{R}}=40{ }^{\circ} \mathrm{C}\right)$. The calcium response of Gl $\gamma$ was maximal when stimulated with the lowest temperature $\Delta \mathrm{T}_{\mathrm{p}}=-3.2 \pm 0.2{ }^{\circ} \mathrm{C}\left(\mathrm{T}_{\mathrm{R}}=0{ }^{\circ} \mathrm{C}\right)$ as shown in Figure $3.11 \mathrm{~A}$. With increasing Ringer temperature, the response decreased (Figure 3.11 B and C) and was not detectable with the control stimulus $\Delta \mathrm{T}_{\mathrm{p}}=0 \pm 0.2{ }^{\circ} \mathrm{C}\left(\mathrm{T}_{\mathrm{R}}=25{ }^{\circ} \mathrm{C}\right)$. Stimulation with warm temperature $\Delta \mathrm{T}_{\mathrm{p}}=+1.6 \pm 0.2{ }^{\circ} \mathrm{C}\left(\mathrm{T}_{\mathrm{R}}=40{ }^{\circ} \mathrm{C}\right)$ did not elicit detectable responses in Gl $\gamma$ either (Figure $3.11 \mathrm{E}$ ).

To compare the responses of Gl $\gamma$ to graduated temperature stimulations, the maximal $\Delta \mathrm{F} / \mathrm{F}$ of measurements performed with this preparation were plotted against $\Delta \mathrm{T}_{\mathrm{p}}$ in a scatter plot (Figure $3.11 \mathrm{G}$ ). This plot shows a linearly increasing response to decreasing temperatures and no significant change of maximal $\Delta \mathrm{F} / \mathrm{F}$ for rising temperatures. In order to test for inhibitory responses, the minimal $\Delta \mathrm{F} / \mathrm{F}$ was calculated for the experiments using warm temperature. The results were virtually identical to the data shown in Figure $3.11 \mathrm{G}$. To exclude that this might be due to inferior properties of fluo-4 for the detection of inhibitory responses, the result was confirmed using rhod $10 \mathrm{kDa}$ dextran and Oregon Green 488 BAPTA-1 $10 \mathrm{kDa}$ dextran (data not shown).

Corresponding results were achieved with five preparations stimulated with temperatures from $0{ }^{\circ} \mathrm{C}$ to $50{ }^{\circ} \mathrm{C}$. The response using low temperatures included the complete structure of Gl $\gamma$ in all measured specimens. In all cases the responses of Gl $\gamma$ increased apparently linearly with decreasing temperature. There was no detectable response upon stimulation with control and high temperature. No saturation of the response was detectable in all five scatter plots. The coldest stimulus used in these 

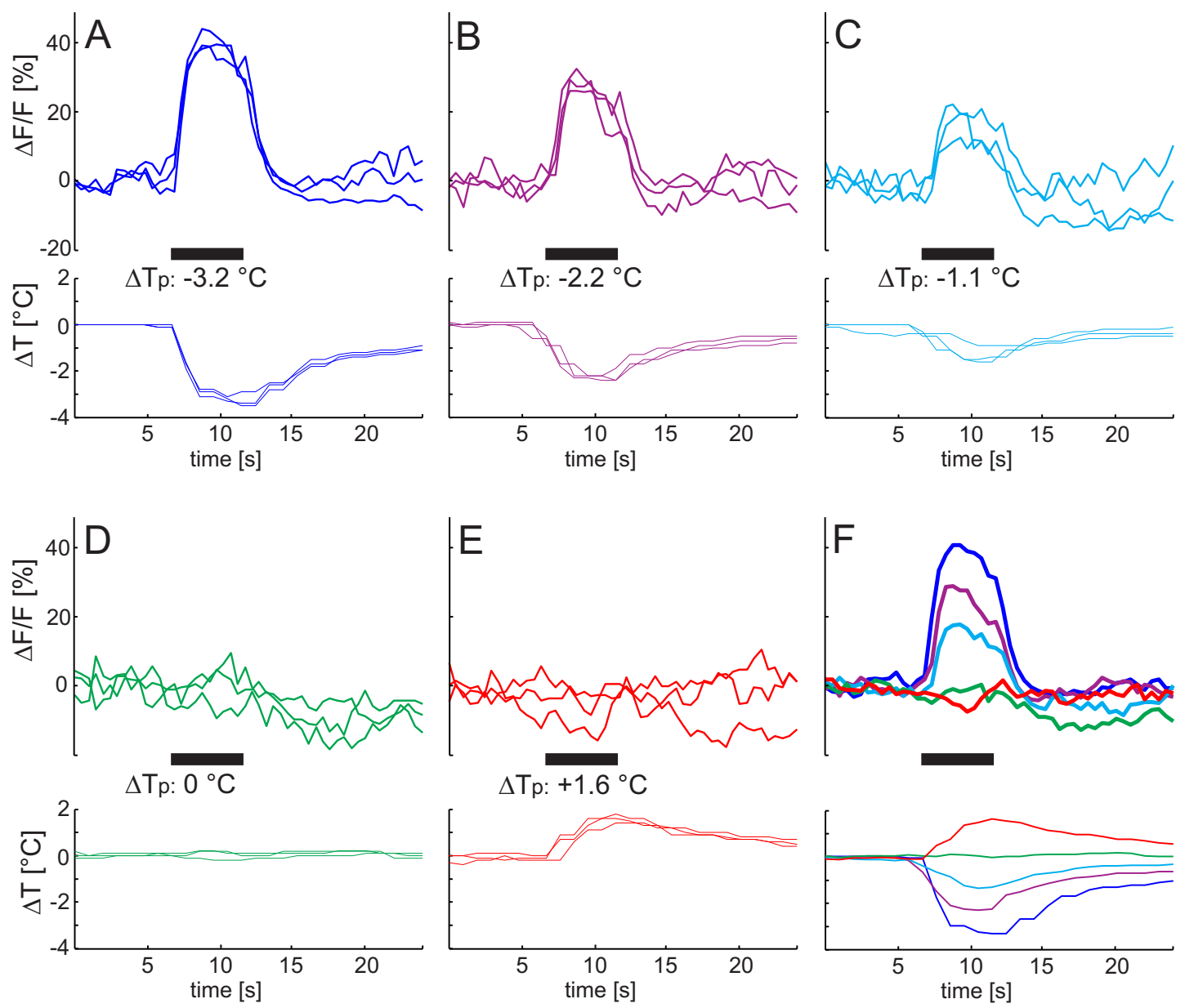

$\Delta \mathrm{Tp}:+1.6^{\circ} \mathrm{C}$

G
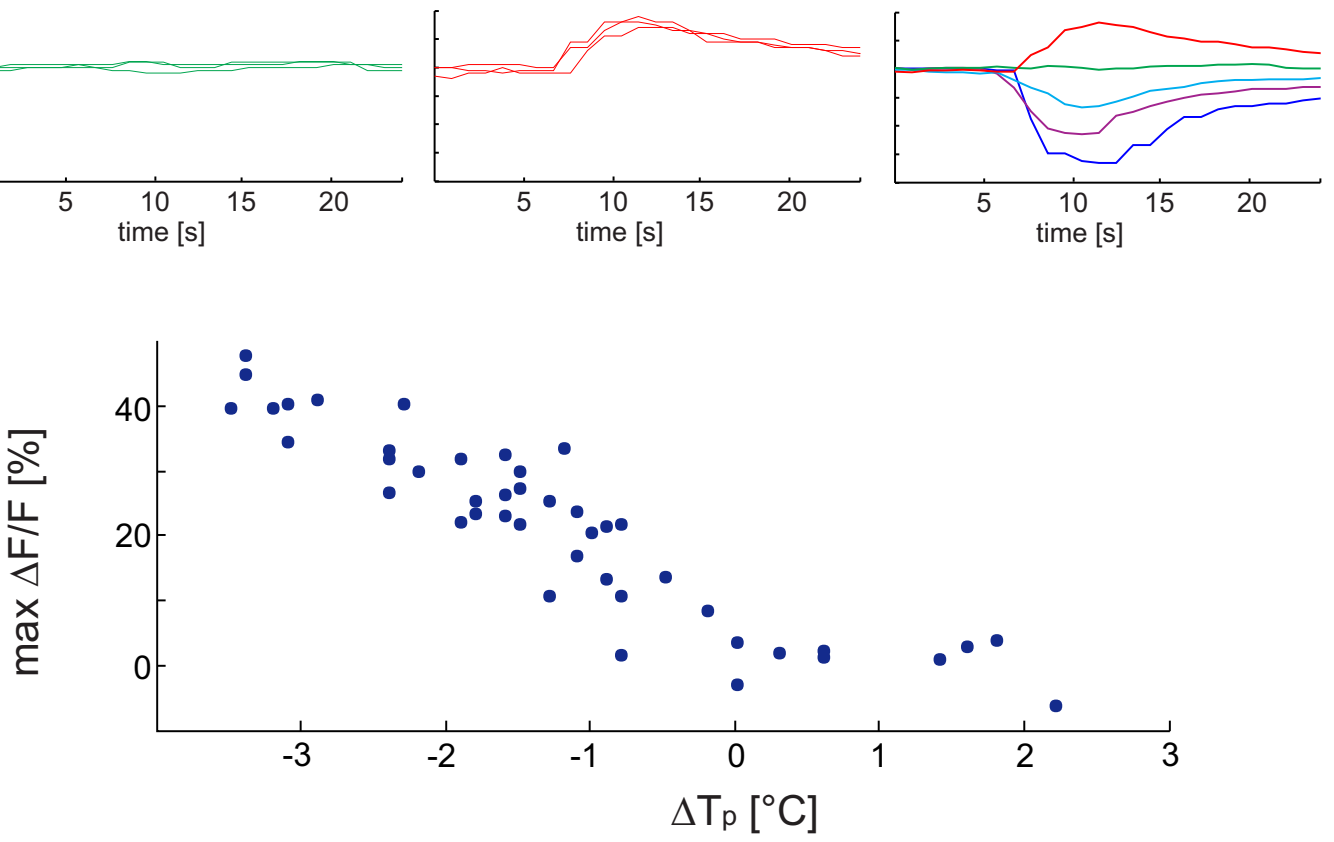

Figure 3.11: Responses of ipsilateral presynaptic terminals of Gl $\gamma$ to temperature stimulation

A to $\mathbf{E}: \Delta \mathrm{F} / \mathrm{F}$ time traces of $\mathrm{Gl} \gamma$ with corresponding $\Delta \mathrm{T}$ time traces upon temperature stimulation. Different temperatures of pipetted Ringer solution used for stimulations are presented with three typical traces each. The time intervals of the temperature stimulus application are visualised with a black line. A: $\Delta \mathrm{T}_{\mathrm{p}}=-3.2 \pm 0.2{ }^{\circ} \mathrm{C}\left(\mathrm{T}_{\mathrm{R}}=0{ }^{\circ} \mathrm{C}\right)$
B: $\Delta \mathrm{T}_{\mathrm{p}}=-2.2 \pm 0.3{ }^{\circ} \mathrm{C}\left(\mathrm{T}_{\mathrm{R}}=8{ }^{\circ} \mathrm{C}\right)$
$\mathrm{C}: \Delta \mathrm{T}_{\mathrm{p}}=-1.1 \pm 0.4^{\circ} \mathrm{C}\left(\mathrm{T}_{\mathrm{R}}=15^{\circ} \mathrm{C}\right)$
D: $\Delta \mathrm{T}_{\mathrm{p}}=0 \pm 0.2{ }^{\circ} \mathrm{C}\left(\mathrm{T}_{\mathrm{R}}=25^{\circ} \mathrm{C}\right)$
$\mathrm{E}: \Delta \mathrm{T}_{\mathrm{p}}=1.6 \pm 0.2{ }^{\circ} \mathrm{C}\left(\mathrm{T}_{\mathrm{R}}=40^{\circ} \mathrm{C}\right)$.

F: Overlay of mean $\Delta \mathrm{F} / \mathrm{F}$ time traces with corresponding mean $\Delta \mathrm{T}$ time traces presented in $\mathrm{A}$ to $\mathrm{E}$.

G: Scatter plot of response strength (maximal $\Delta \mathrm{F} / \mathrm{F}$ ) to $\Delta \mathrm{T}_{\mathrm{p}}$ for all measurements with this specimen. 
experiments achieved a temperature decrease of $\Delta \mathrm{T}_{\mathrm{p}}=-3.2 \pm 0.2{ }^{\circ} \mathrm{C}\left(\mathrm{T}_{\mathrm{R}}=0{ }^{\circ} \mathrm{C}\right)$. This temperature decrease was apparently not sufficient to achieve a saturation of the Gly response.

Gl $\gamma$ is not only innervated by OSN axons emerging from the ipsilateral OE but also by deviating OSN axons. The intriguing question was: are deviating OSN axons also temperature sensitive? The experiments that were performed to answer this question are presented in the following section.

\subsubsection{Temperature sensitivity of deviating axons of the olfactory sensory neurons}

To explore the temperature sensitivity of deviating OSN axons, the right OB was imaged. The olfactory sensory neuron axons emerging from the right $\mathrm{OE}$ were traced with Alexa Fluor $56810 \mathrm{kDa}$ dextran and were used to locate Gl $\gamma$ within the right OB. The deviating OSN axons emerging from the left OE were traced with fluo-4 $10 \mathrm{kDa}$ dextran.

The fluorescence of fluo-4 at basal calcium levels within vital OSN axons was barely detectable. It was not possible to verify a successful tracing of the deviating OSN axons in advance of the actual experiment. Additionally, it was not possible to exclude preparations with damaged deviating OSN axons from the experiments since no alternative stimulation to test the vitality of the deviating OSN axons was available.

A total of 13 animals were scanned for responses to temperature stimulation of the contralateral OE. Only in four preparations a calcium response within Gl $\gamma$ could be detected (Figure 3.12). The residual nine preparations most probably included specimens with failed tracing of the deviating OSN axons (see previous chapter 3.1.3) and specimens with successfully traced deviating OSN axons that were subsequently damaged during the preparation.

The images presented in Figure 3.12 A and B show the morphology of the ipsilateral olfactory projection that was used to localise Gl $\gamma$. The basal fluorescence of fluo-4 in the deviating OSN axons was not detectable. The signal from deviating OSN axons was only visible at high calcium concentrations during spontaneous activity or during response to stimulation with cold Ringer solution. This response signal was spatially visualised in two different ways: a 'pixel correlation map' and spatial map of $\Delta F / F$ at response peak. The 'pixel correlation map' was calculated for a time series of each single experiment to analyse the spatial distribution of the coherent activity within the 
measured optical slice (Figure 3.12 C). The overlay of the 'pixel correlation map' with the ipsilateral tracing showed that the transient $\left[\mathrm{Ca}^{2+}\right]_{\mathrm{i}}$ response was restricted to the area of Gl $\gamma$. This corresponds to morphological tracings of deviating OSN axons as presented in chapter 3.1. The spatial map of $\Delta \mathrm{F} / \mathrm{F}$ at response peak (Figure $3.12 \mathrm{E}$ ) verified that the deviating OSN axons coherently responded to the cold temperature stimulus. Typical time courses of $\Delta \mathrm{F} / \mathrm{F}$ calcium response to stimulation with different temperatures are presented in Figure $3.12 \mathrm{~F}$ to $\mathrm{H}$. A clear response of the deviated OSN axons during stimulation with cold Ringer solution could be reproduced three times for all four preparations. Stimulation with warm Ringer solution and negative control stimulation failed to elicit a response.

These results for deviating OSN axons correspond with responses of ipsilateral OSN axons within Gl $\gamma$ presented in the previous section. In both cases the stimulation with cold Ringer solution resulted in a clear response. The stimulation with warm or roomtemperature Ringer solution did not elicit a response. 

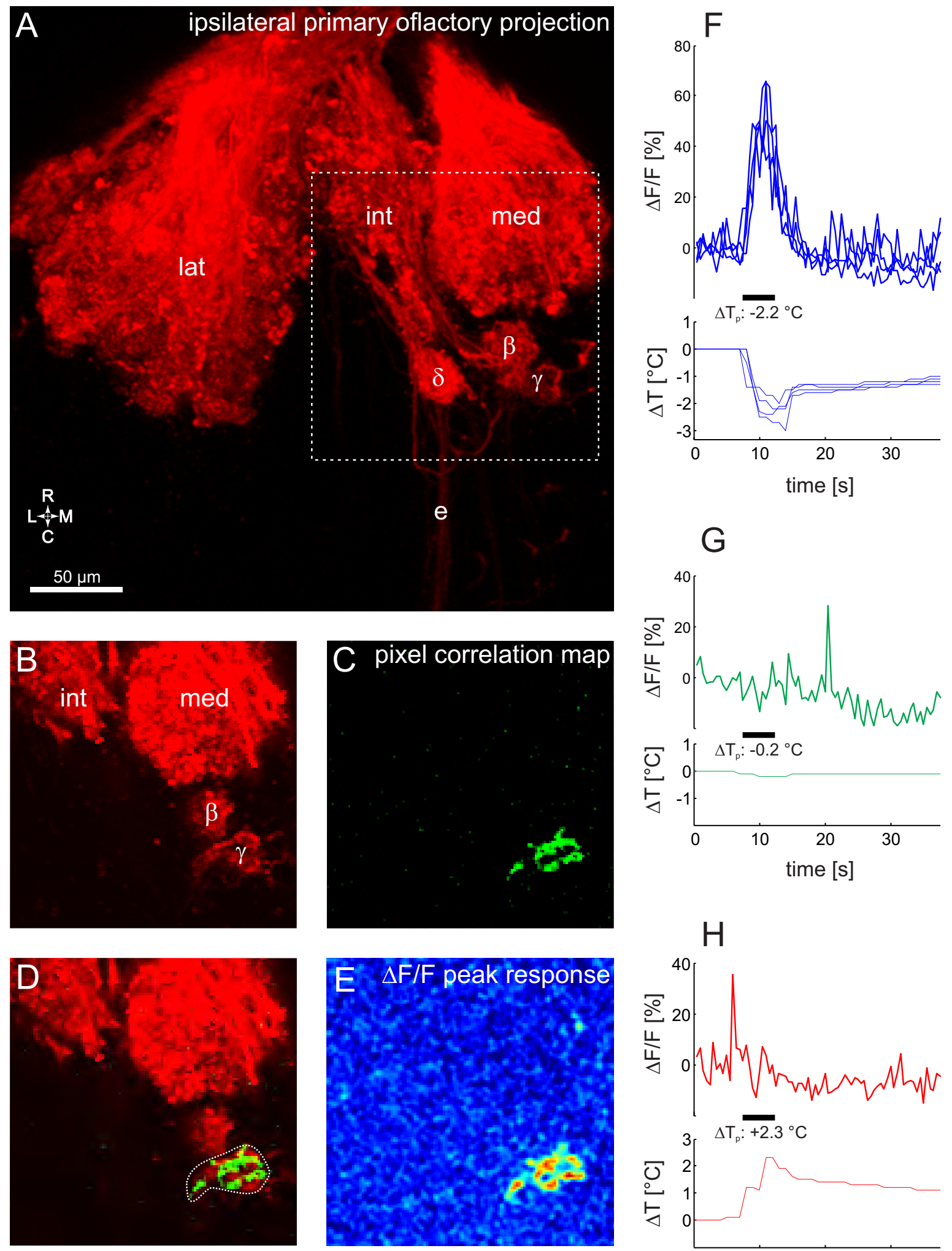

$\Delta \mathrm{F} / \mathrm{F}-5 \%$ $30 \%$

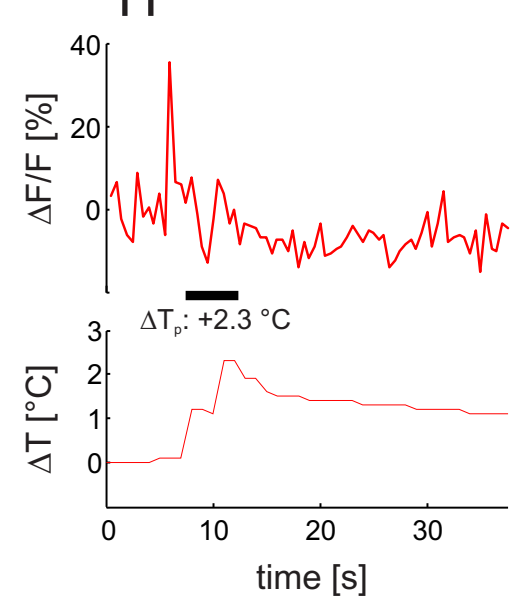

Figure 3.12: Temperature sensitivity of deviating axons of olfactory sensory neurons 


\subsubsection{Summary: calcium imaging experiments with axons of olfactory sensory neurons}

The calcium imaging revealed a sensitivity of Gl $\gamma$ to stimulation of the OE with cold Ringer solution within both OSN axon populations that constitute Gly: ipsilateral OSN axons as well as deviating OSN axons. The sensitivity of OSN axons to temperature changes was not ubiquitous since G1 $\beta$ innervating OSN axons were not temperature sensitive but responded specifically to amino acids, forskolin and mechanical stimulation. These experiments verify that a change in temperature elicits a transient $\left[\mathrm{Ca}^{2+}\right]_{\mathrm{i}}$ response within Gl $\gamma$ innervating OSN axons. The experiments in the next chapter address the question of whether the response to temperature stimulation is relayed through the first olfactory synapse.

Figure 3.12: Temperature sensitivity of deviating axons of olfactory sensory neurons

A: Dorsoventral MIP of $47 \mu \mathrm{m}$ thick scanned volume as overview of OSN projections within the ventral OB. Signal from OSN axons emerging from the ipsilateral OE is presented in the red channel.

B: Single optical slice from the boxed area in A used for the time series acquisition.

C: Pixel correlation map of a single response to $\Delta \mathrm{T}_{\mathrm{p}}=-2.5^{\circ} \mathrm{C}\left(\mathrm{T}_{\mathrm{R}}=0{ }^{\circ} \mathrm{C}\right)$ stimulation at contralateral OE.

D: Overlay of B and C. ROI used to compute response traces presented in $\mathrm{F}$ to $\mathrm{H}$ marked with a dotted line.

E: Peak response to a single stimulation experiment $\Delta \mathrm{T}_{\mathrm{p}}=-2.5^{\circ} \mathrm{C}\left(\mathrm{T}_{\mathrm{R}}=0{ }^{\circ} \mathrm{C}\right)$ as $\Delta \mathrm{F} / \mathrm{F}$ with corresponding colorbar.

$\mathbf{F}$ to $\mathbf{H}$ : Four typical $\Delta \mathrm{F} / \mathrm{F}$ response traces to temperature stimulations with corresponding $\Delta \mathrm{T}$ traces upon stimulation with $\Delta \mathrm{T}_{\mathrm{p}}=-2.2 \pm 0.5^{\circ} \mathrm{C}\left(\mathrm{T}_{\mathrm{R}}=0{ }^{\circ} \mathrm{C}\right)$ in $\mathrm{F}$ and one trace upon stimulation with $\Delta \mathrm{T}_{\mathrm{p}}=-0.2{ }^{\circ} \mathrm{C}\left(\mathrm{T}_{\mathrm{R}}=25^{\circ} \mathrm{C}\right)$ in $\mathrm{G}$ and $\Delta \mathrm{T}_{\mathrm{p}}=+2.3{ }^{\circ} \mathrm{C}$ $\left(\mathrm{T}_{\mathrm{R}}=50^{\circ} \mathrm{C}\right)$ in $\mathrm{H}$ each. The time intervals of the temperature stimulus application are visualised with a black line.

Abbreviations: $\beta, \mathrm{Gl} \beta ; \gamma, \mathrm{Gl} \gamma ; \delta$, IS $\delta$; e, EBOF; int, intermediate cluster; lat, lateral cluster; med, medial cluster. 


\subsection{Calcium imaging of processes and somata of mitral cells}

The evaluation of dye distribution within the glomerular structures after bulk staining of the OB with AM coupled calcium sensitive dyes revealed that this staining is specific for the postsynaptic glomerular neuropil. It was used to study the responses within the postsynaptic neuropil of Gl $\gamma$ and somata of mitral cells to temperature stimulation. All following experiments were performed using stage $51-53$ tadpoles.

\subsubsection{Determination of the calcium sensitive dye distribution within the olfactory bulb after bulk staining}

\subsubsection{Specific staining of postsynaptic glomerular neuropil with Fura Red/AM}

Fura Red was chosen as a fluorophore for the evaluation of the dye distribution after bulk staining of the OB with an AM coupled dye as it has a strong emission at low $\left[\mathrm{Ca}^{2+}\right]$ levels.

Four tadpoles were electroporated with Alexa $48810 \mathrm{kDa}$ dextran in both nasal cavities to stain OSN axons within olfactory glomeruli. The explanted nose-olfactory bulb preparation was then stained with Fura Red/AM by bolus loading. Figure 3.13 clearly shows that the fluorescence of Fura Red was complementary to the fluorescence of Alexa Fluor $48810 \mathrm{kDa}$ dextran.

\section{Figure 3.13: Fluorescence distribution after bolus loading with Fura Red/AM}

The fluorescence from the Alexa Fluor 488 fluorophore is presented in the green channel. The fluorescence from the Fura Red fluorophore is presented in the red channel.

A to C: Dorsoventral MIP of $26.5 \mu \mathrm{m}$ thick scanned volume as overview of ventral part of medial cluster. The traced OSN axons are clearly visible by Alexa Fluor 488 fluorescence in A. The somata of $\mathrm{OB}$ neurons and their neuropils can be clearly distinguished in the fluorescence signal of the Fura Red fluorophore that was applied through bolus loading. An example of a mitral cell dendrite that enters the glomerular layer is marked with an arrow in B. No axons of OSNs that enter the glomerular layer from the rostral direction are visible within the Fura Red signal. The overlay of both channels in C shows some apparent overlap caused by MIP, as pointed out by a single optical slice (D to F) from the boxed area in A.

D to F: Single optical slice through a glomerular structure. Example of prominent axonal compartment (D) that lacks Fura Red signal (E) and is thus complementary to the red channel $(\mathrm{F})$ is marked with an arrowhead.

Abbreviations: C, caudal; L, lateral; M, medial; R, rostral. 

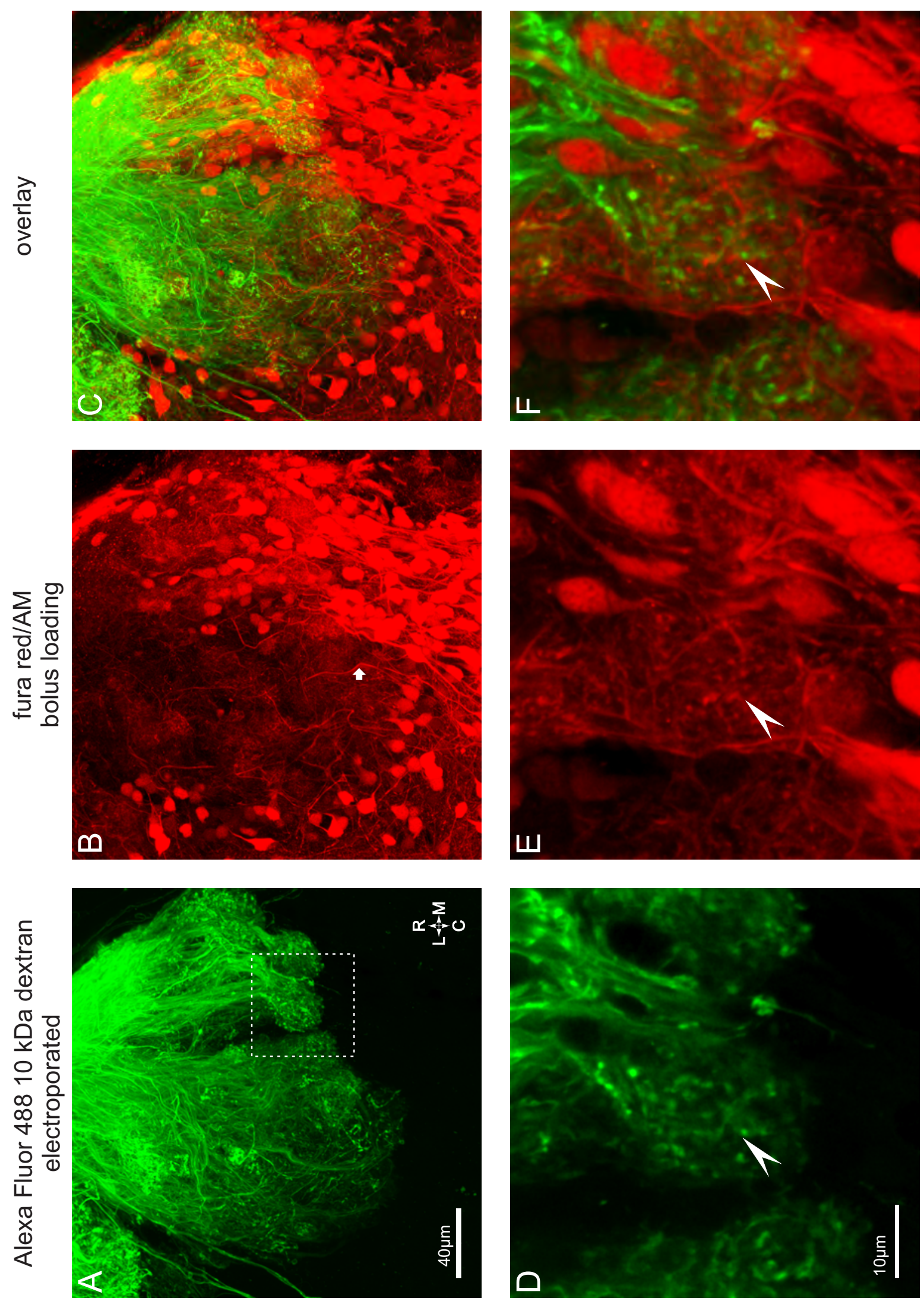

Figure 3.13: Fluorescence distribution after bolus loading with Fura Red/AM 
To verify that the specific staining of $\mathrm{OB}$ neurons was not due to an insufficient availability of AM coupled dye using the bolus loading approach, this experiment was repeated using bath incubation of sliced nose-olfactory bulb preparations with the AM coupled dye. The bath application of Fura Red/AM assured an abundance of the AM coupled dye at the sliced surface of the OB. The staining pattern of the OB after bath incubation with Fura Red/AM was consistent with the results of bolus loading (Figure $3.14 \mathrm{~A}$ to $\mathrm{C}$ ) and indicated that the absence of staining within OSN axons was not caused by limited availability of the injected dye. The specific staining of the OB neurons and their glomerular neuropil after bath incubation with Fura Red/AM was confirmed in five preparations.

The possibility that electroporated Alexa Fluor $48810 \mathrm{kDa}$ dextran interfered with dye loading of Fura Red/AM was investigated by a control experiment. Three tadpoles were electroporated with Alexa Fluor $48810 \mathrm{kDa}$ dextran in both nasal cavities. The OE of these animals was sliced and bulk stained with Fura Red/AM using bath incubation. Subsequent imaging of this OE preparation showed OSN somata that were stained by both dyes in varying portions. This staining pattern indicates that electroporation with Alexa Fluor $48810 \mathrm{kDa}$ dextran did not interfere with the subsequent bulk staining of OSNs by Fura Red/AM (Figure 3.14 D to F).

\section{Figure 3.14: Fluorescence distribution after bath incubation with Fura Red/AM}

A to $\mathbf{C}$ : Single optical slice through a glomerular structure of the sliced nose-olfactory bulb preparation. Green channel presents the signal from OSN axons that were traced by electroporation of OE with Alexa Fluor $48810 \mathrm{kDa}$ dextran in A. Red channel presents fluorescence signal from Fura Red that was applied as AM coupled dye in bath Ringer in B. Overlay of both predominantly complementary signals presented in C. Arrowheads mark examples of OSN axons that are clearly visible by their Alexa Fluor 488 fluorescence but were not stained with Fura Red.

D to F: Control experiment showing sliced OE after Alexa Fluor $48810 \mathrm{kDa}$ dextran electroporation and subsequent incubation in Fura Red/AM bath solution. Green channel presents the signal from Alexa Fluor $48810 \mathrm{kDa}$ dextran dye in D. Red channel presents the signal from Fura Red dye in E. Overlay of both channels is presented in F. Representative examples of OSN somata that are stained by both applicated dyes are marked with an asterisk. An example of OSN soma that is stained only by Alexa Fluor $48810 \mathrm{kDa}$ dextran is marked with a triangle. An example of OSN soma that is stained only by Fura Red is marked with a square.

Abbreviations: pc, principal cavity; C, caudal; L, lateral; M, medial; R, rostral. 

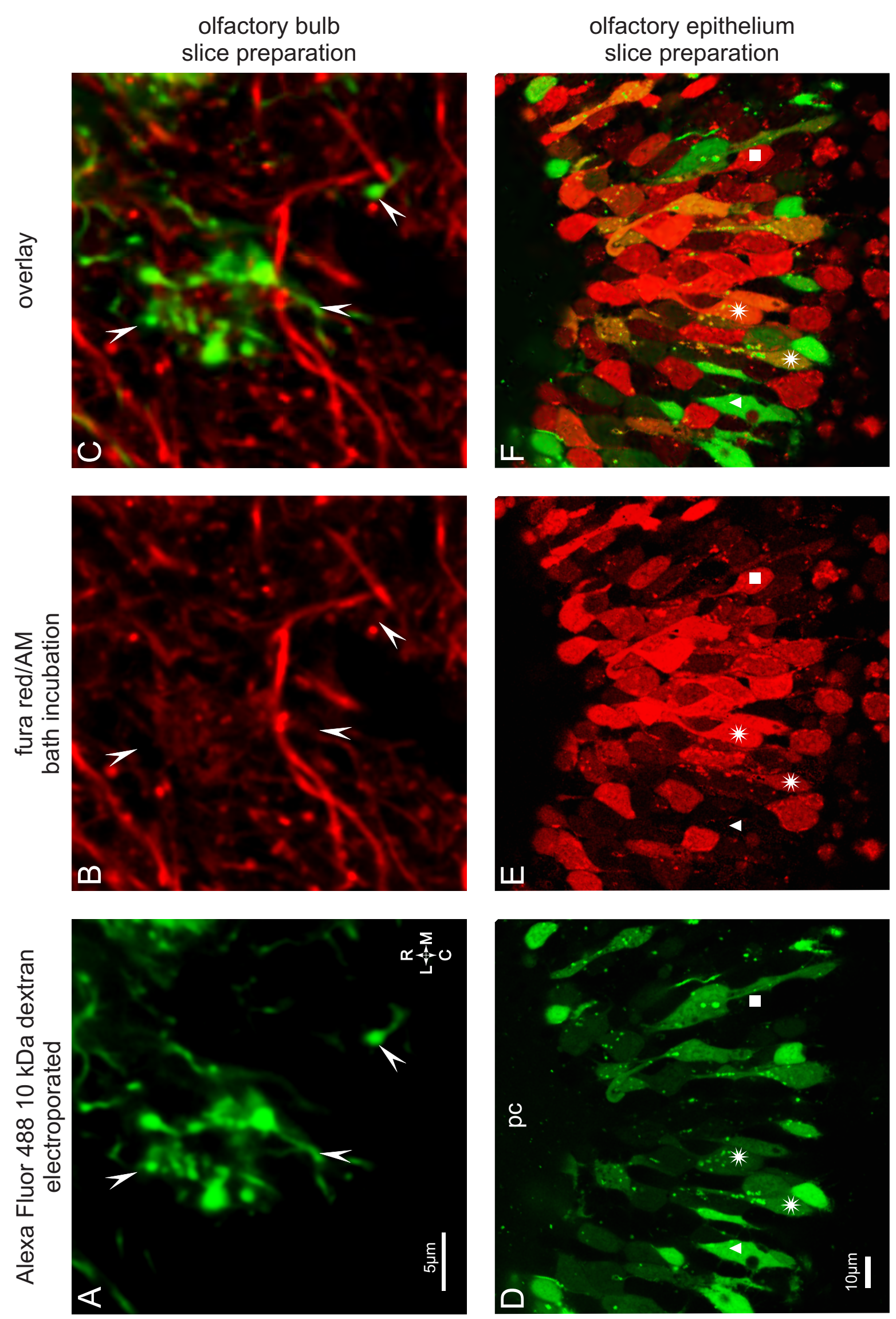

Figure 3.14: Fluorescence distribution after bath incubation with Fura Red/AM 
The absence of AM coupled dye in OSN axons indicates a selective staining of OB neurons and their neuropil by application of AM conjugated dyes. Since fluo-4 was used to measure transient $\left[\mathrm{Ca}^{2+}\right]_{\mathrm{i}}$ responses, it was necessary to evaluate the staining pattern of this dye as well.

\subsubsection{Specific staining of postsynaptic glomerular neuropil with fluo-4/AM}

The evaluation of the dye distribution after bulk staining of the OB was performed for bolus loading (three preparations) and bath incubation (four preparations) with fluo-4/AM. The experimental animals were electroporated with Alexa Fluor 56810 kDa dextran in both nasal cavities prior to preparation.

The spatial distribution of fluorescence was analysed with two different methods. The $\Delta \mathrm{F} / \mathrm{F}$ for every single pixel was calculated. Examples are displayed as peak response $\Delta \mathrm{F} / \mathrm{F}$ spatial map in Figure 3.15 D and Figure 3.16 D. The highlighted regions in the peak response $\Delta \mathrm{F} / \mathrm{F}$ map were compared with the distribution of traced OSN axons within the optical slice. No overlap between transient $\left[\mathrm{Ca}^{2+}\right]_{\mathrm{i}}$ responses and OSN axon structures could be found. The peak response $\Delta \mathrm{F} / \mathrm{F}$ map showed the fluorescence increase for a single time point of the whole time series. Additionally, a 'pixel correlation map' was calculated from the complete time series to analyse the distribution of physiologically relevant fluorescence signals (Manzini et al., 2007a; methods section 2.6.2). To ensure the applicability of the 'pixel correlation map', the time series scans were performed at high spatial resolution. The overlay of the 'pixel correlation map' with traced OSN axons in Figure 3.15 $\mathrm{C}$ and Figure $3.16 \mathrm{C}$ shows that both are complementary.

These results indicate that transient $\left[\mathrm{Ca}^{2+}\right]_{\mathrm{i}}$ responses measured within the olfactory glomeruli originate completely from the postsynaptic neuropil after bulk staining of the OB with fluo-4/AM. 

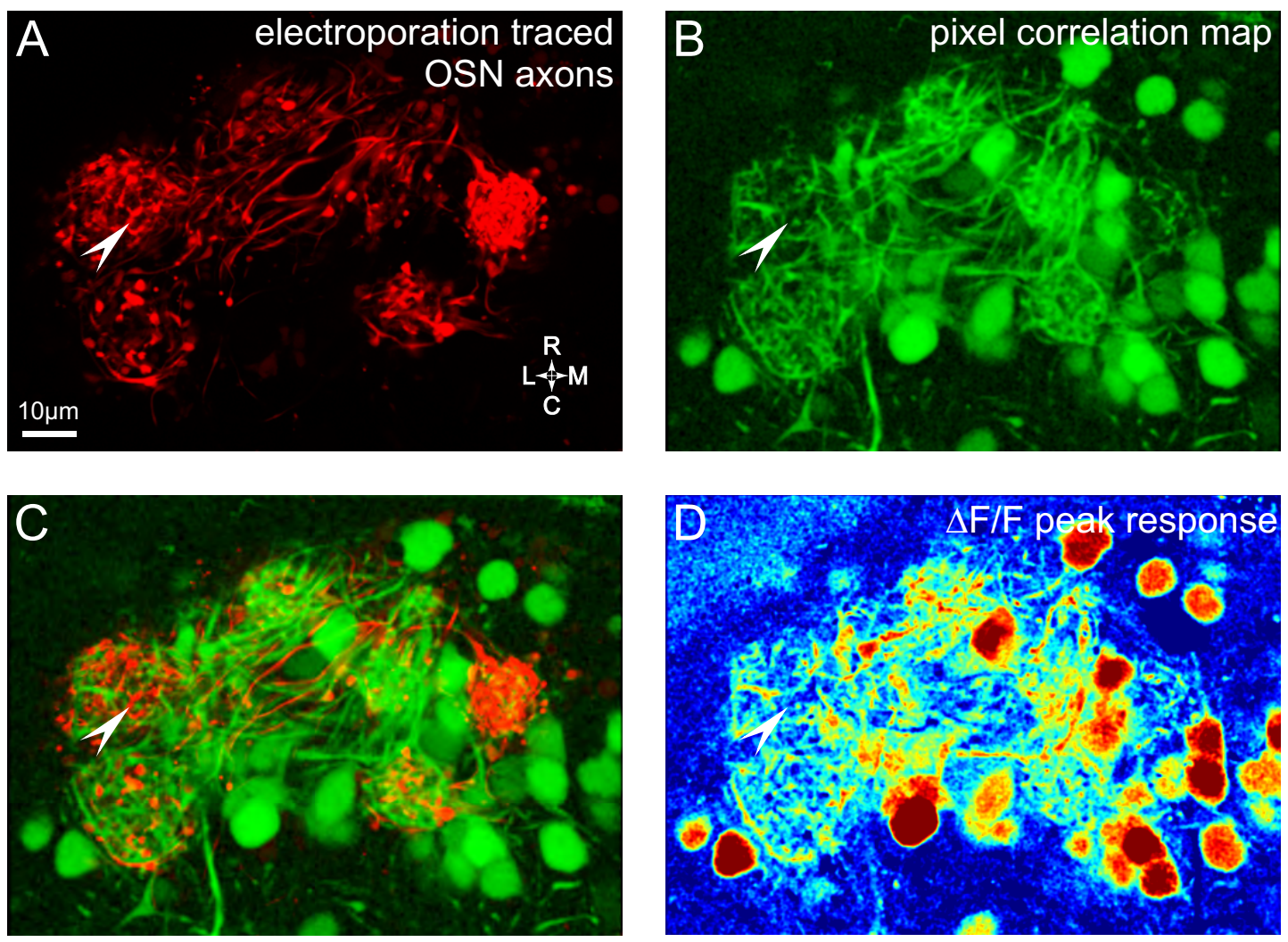

$\Delta \mathrm{F} / \mathrm{F}-5 \%$ $40 \%$

\section{Figure 3.15: Fluorescence distribution after bolus loading with fluo-4/AM}

A: Presynaptic glomerular tufts formed by OSN axons traced with Alexa Fluor 56810 kDa dextran.

B: 'Pixel correlation map' calculated from the fluo-4 fluorescence signal upon stimulation with amino acid mixture.

C: Overlay of traced OSN axons and 'pixel correlation map'.

D: Peak response $\Delta \mathrm{F} / \mathrm{F}$ image upon stimulation with amino acid mixture.

Example of a prominent OSN axon that does not show any fluo-4 signal is marked with an arrowhead.

Abbreviations: C, caudal; L, lateral; M, medial; R, rostral. 

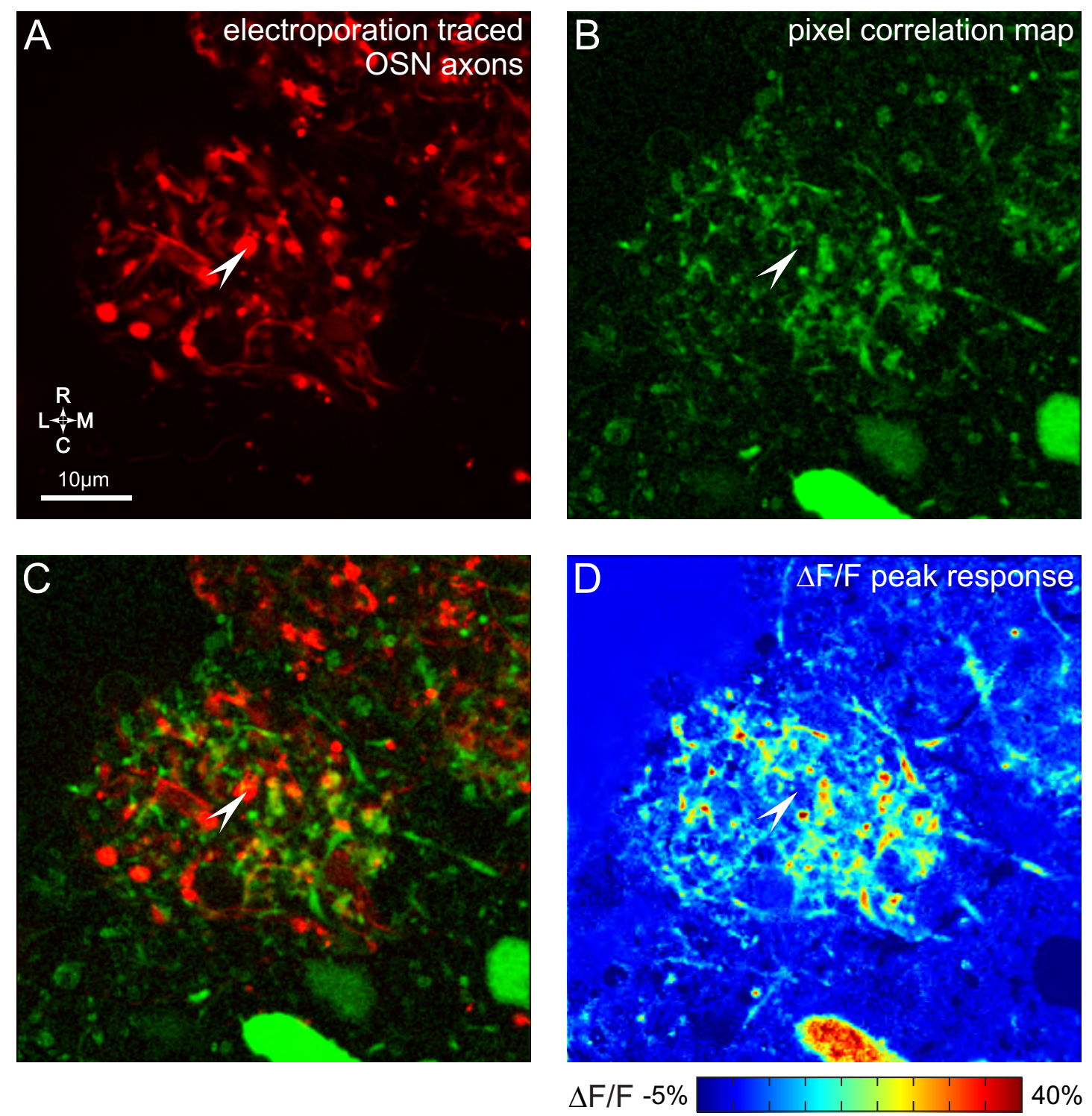

Figure 3.16: Fluorescence distribution after bath incubation with fluo-4/AM

A: Presynaptic glomerular tuft formed OSN axons traced with Alexa Fluor 56810 kDa dextran.

B: 'Pixel correlation map' calculated from the fluo-4 fluorescence signal of a single experiment.

C: Overlay of traced OSN axons and 'pixel correlation map'.

D: Peak response $\Delta \mathrm{F} / \mathrm{F}$ image.

Example of a prominent OSN axon that does not show any fluo-4 signal is marked with an arrowhead.

Abbreviations: C, caudal; L, lateral; M, medial; R, rostral. 


\subsubsection{Conclusions for application of AM coupled calcium sensitive dyes in the OB}

Neither a basal fluorescence signal nor physiological $\left[\mathrm{Ca}^{2+}\right]_{\mathrm{i}}$ transients could be detected in traced OSN axons within the OB after bulk staining with AM coupled, calcium sensitive dyes. Hence the bolus loading of fluo-4/AM in the OB can be used for calcium imaging selectively from postsynaptic glomerular neuropil. 


\subsubsection{Response profiles of mitral cell somata and their processes upon temperature stimulation}

The bolus loading of the OB with fluo-4/AM was used to study the responses of the processes and somata of mitral cells to temperature stimulation.

\subsubsection{Localisation of temperature sensitive dendrites within Gly}

The first aim of these experiments was to find Gly in a bulk stained preparation and to verify that the response of OSNs to temperature stimulation is relayed through the first olfactory synapse. To accelerate the recognition of Gl $\gamma$, the contralateral nasal cavity was electroporated with Alexa Fluor $56810 \mathrm{kDa}$ dextran in advance. At the beginning of the experiment the position of Gl $\gamma$ was located by finding the innervation area of deviating OSN axons (Figure 3.17 B). Application of cold Ringer solution as temperature stimulus revealed a temperature sensitive glomerular structure within the imaged area in all five imaged OBs (Figure 3.17 F). This temperature sensitive neuropil was contiguous with deviating OSN axons that served for the localisation of Gly (Figure $3.17 \mathrm{G})$. Additional to neuropil structures, visible as a filamentary meshwork, globular structures of about $10 \mu \mathrm{m}$ diameter responded to the temperature stimulus (Figure 3.17 F, closed arrowhead). These structures were presumed to be mitral cell somata.

Thus, the tracing of deviating OSN axons by electroporation of the contralateral nasal cavity can be used for localisation and identification of temperature sensitive neuropil within Gly in bulk stained preparations. 

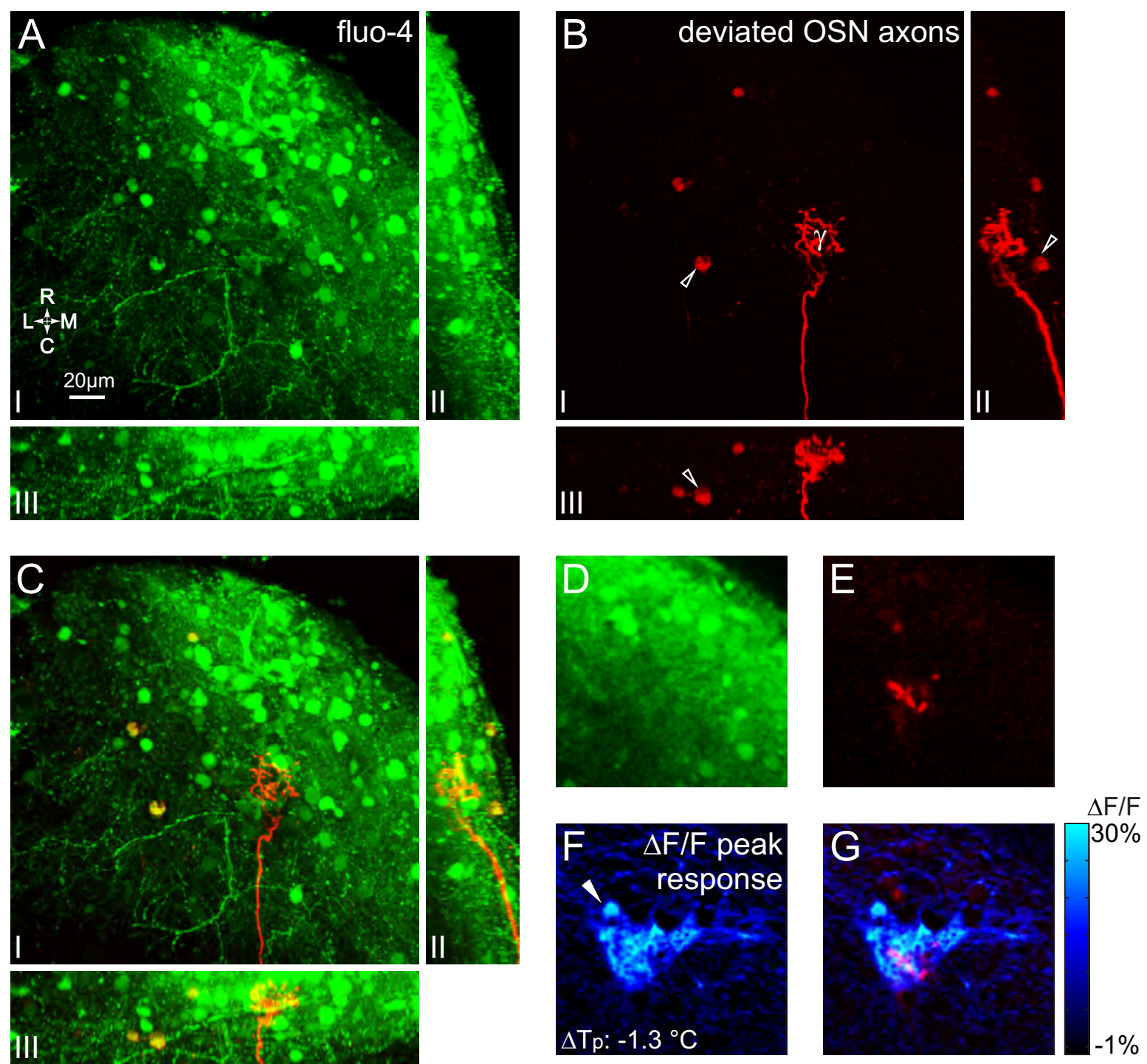

Figure 3.17: Temperature sensitivity of postsynaptic terminals of Gly

A to C: Overview of the ventral OB after bolus loading with fluo-4/AM (A). Deviating OSN axons emerging from the contralateral OE are traced with Alexa Fluor 568 and presented in the red channel (B). They enter the imaged volume at the ventrocaudal rim, protrude rostro-dorsally and form a glomerular tuft in the area of Gl $\gamma$. Location of Gl $\gamma$ at the ventral border of the $\mathrm{OB}$ is visible in the overlay of lateral and frontal projections in $\mathrm{C}_{\text {II }}$ and $\mathrm{C}_{\text {III. }}$ An example of amoeboid structures that are visible in both channels and presumably represent microglia is marked with an open arrowhead in B.

D to G: Single optical slices from the imaged area. Fluo-4 fluorescence signal at the beginning of the experiment is presented in D. Fluorescence signal from deviating OSN axon terminals is presented in the red channel $(\mathrm{E})$. Response to temperature stimulus $\Delta \mathrm{T}_{\mathrm{p}}=-1.3{ }^{\circ} \mathrm{C}\left(\mathrm{T}_{\mathrm{R}}=0{ }^{\circ} \mathrm{C}\right)$ is presented in $\mathrm{F}$ as $\Delta \mathrm{F} / \mathrm{F}$ at response peak. The respective colormap covers only the blue and green channels. The contiguity of temperature sensitive neuropil and deviating OSN axon terminals is visible in G. The presumed mitral cell soma is marked with a closed arrowhead in F.

Abbreviations: $\gamma$, Gl $\gamma, \mathrm{C}$, caudal; L, lateral; M, medial; R, rostral. 


\subsubsection{Response profiles of mitral cell somata and postsynaptic Gly neuropil upon graduated temperature stimulation}

The relationship between the response strength of mitral cell somata or postsynaptic Gly neuropil and intensity of the applied temperature stimulus was studied by application of Ringer solution at temperature $\mathrm{T}_{\mathrm{R}}$ of $0-50{ }^{\circ} \mathrm{C}$. Ringer solution at room temperature $\left(24-26{ }^{\circ} \mathrm{C}\right)$ served as a negative control. Typical responses within the optical slice are presented in Figure 3.18 as spatial maps of $\Delta \mathrm{F} / \mathrm{F}$ peak response.

For a detailed analysis of the acquired time series, the image area that contained the temperature-sensitive meshwork was outlined as a single ROI. The responding mitral cell somata were each outlined as single ROI. The major part $(82 \%, 14$ cells from five preparations) of temperature sensitive mitral cells responded exclusively to cold temperature stimulation with an increased response to colder stimuli. No response to the negative control stimulation or the stimulation with warm Ringer solution was detectable in these mitral cells. Two typical examples are marked in Figure 3.18 as mitral cells 1 and 2 . Their response traces and scatter plots of maximal $\Delta \mathrm{F} / \mathrm{F}$ to $\Delta \mathrm{T}_{\mathrm{p}}$ from all measurements are presented in Figure 3.19 A and B.

Additionally three mitral cells $(18 \%)$ exclusively responded to warm temperature stimulation. Two examples are marked in Figure 3.18 as mitral cell 3 and 4. Representative response traces of these mitral cells and a scatter plot of maximal $\Delta \mathrm{F} / \mathrm{F}$ to $\Delta \mathrm{T}_{\mathrm{p}}$ are presented in Figure 3.19 $\mathrm{C}$ and $\mathrm{D}$. The somata of these mitral cells responded apparently only to stimulation with temperatures that were higher than room temperature and did not respond to stimulation with low temperature and control stimulation.

\section{Figure 3.18: $\Delta F / F$ peak response maps during stimulation with different temperatures within the bulk stained olfactory bulb}

$\Delta \mathrm{F} / \mathrm{F}$ peak response maps during stimulation with different temperatures. Two typical responses to repeated stimulation with the same temperature are presented. ROI used to calculate example response traces in Figure 3.19 are marked with a dashed outline.
A: $\Delta \mathrm{T}_{\mathrm{p}}=-5.8 \pm 0.5{ }^{\circ} \mathrm{C}\left(\mathrm{T}_{\mathrm{R}}=0{ }^{\circ} \mathrm{C}\right) . \mathbf{B}: \Delta \mathrm{T}_{\mathrm{p}}=-2.3 \pm 0.2{ }^{\circ} \mathrm{C}\left(\mathrm{T}_{\mathrm{R}}=15{ }^{\circ} \mathrm{C}\right)$.
C: $\Delta \mathrm{T}_{\mathrm{p}}=-1.1 \pm 0.1^{\circ} \mathrm{C}\left(\mathrm{T}_{\mathrm{R}}=21^{\circ} \mathrm{C}\right) . \mathbf{D}: \Delta \mathrm{T}_{\mathrm{p}}=0 \pm 0.1{ }^{\circ} \mathrm{C}\left(\mathrm{T}_{\mathrm{R}}=25^{\circ} \mathrm{C}\right)$.
E: $\Delta \mathrm{T}_{\mathrm{p}}=+4 \pm 0.4{ }^{\circ} \mathrm{C}\left(\mathrm{T}_{\mathrm{R}}=40{ }^{\circ} \mathrm{C}\right)$.

Abbreviations: $\gamma, \mathrm{Gl} \gamma, \mathrm{C}$, caudal; L, lateral; M, medial; R, rostral. 

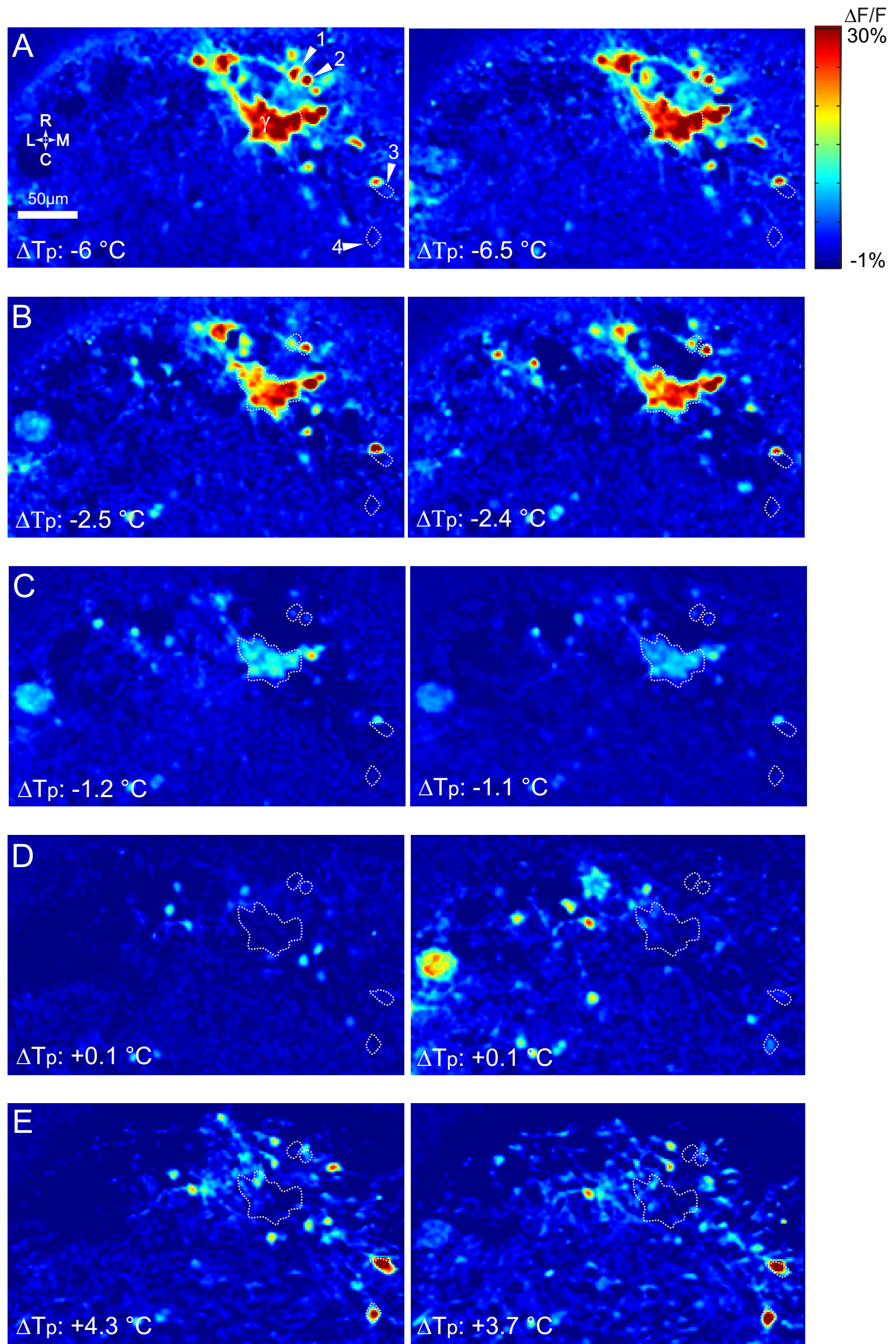

Figure 3.18: $\Delta F / F$ peak response maps during stimulation with different temperatures within the bulk stained olfactory bulb 
The response of the Gl $\gamma$ neuropil meshwork to graded temperature stimulation contained both response patterns: a strong response to cold temperatures and a weaker response to warm temperatures. While a response to cold temperature stimulation was found in all five preparations, an apparent response to warm temperature stimuli was noted in three of them. The control stimulation with room temperature Ringer solution did not elicit a response in the Gl $\gamma$ neuropil meshwork in any preparation.

The spatial response pattern to temperature stimulation is highly coherent for repeated applications. The spatial response pattern to cold temperature stimulation was also coherent between different cold temperatures with gradually decreasing intensity for increasing the stimulus temperature towards room temperature (Figure $3.18 \mathrm{~A}$ to $\mathrm{C}$ ). The spatial distribution of warm temperature responses was different from the responses to cold temperature (Figure $3.18 \mathrm{E}$ ). It consisted of responding mitral cell somata and respective neuropil that were distributed outside as well as within the assumed area of Gl $\gamma$ (marked as $\gamma$ in Figure $3.18 \mathrm{~A}$ ). The dendrites of cold and warm sensitive OB neurons partly intermingled within the Gl $\gamma$ area (with the cold sensitive neuropil being the major fraction) and contributed to the response within Gl $\gamma$. The dendrites of temperature sensitive mitral cells were not restricted to the presumed Gl $\gamma$ area but were also prominent in the surrounding area (an example of prominent dendrites of warm temperature sensitive mitral cells outside of the presumed Gl $\gamma$ area is shown in Figure $3.18 \mathrm{E})$.

After measuring the response upon temperature stimulation of the ipsilateral $\mathrm{OE}$, the same preparations were used to investigate a response to temperature stimuli applied to the contralateral $\mathrm{OE}$ as described in the following section.

\section{Figure 3.19: Response traces to graduated temperature stimulation within the bulk stained olfactory bulb}

Responses of ROIs marked in Figure 3.18 A are presented as one representative time trace for each temperature applied and scatter plot of maximal $\Delta \mathrm{F} / \mathrm{F}$ against $\Delta \mathrm{T}_{\mathrm{p}}$ for all measurements. The time intervals of the temperature stimulus are visualised with a black line below the traces.
A and B: Mitral cell somata responding to cold temperature stimulation.
C and D: Mitral cell somata responding to warm temperature stimulation.
E: Response measured within Gl $\gamma$ neuropil meshwork.
$\mathbf{F}: \Delta \mathrm{T}$ time traces corresponding to $\Delta \mathrm{F} / \mathrm{F}$ time traces presented in $\mathrm{A}$ to $\mathrm{E}$. 

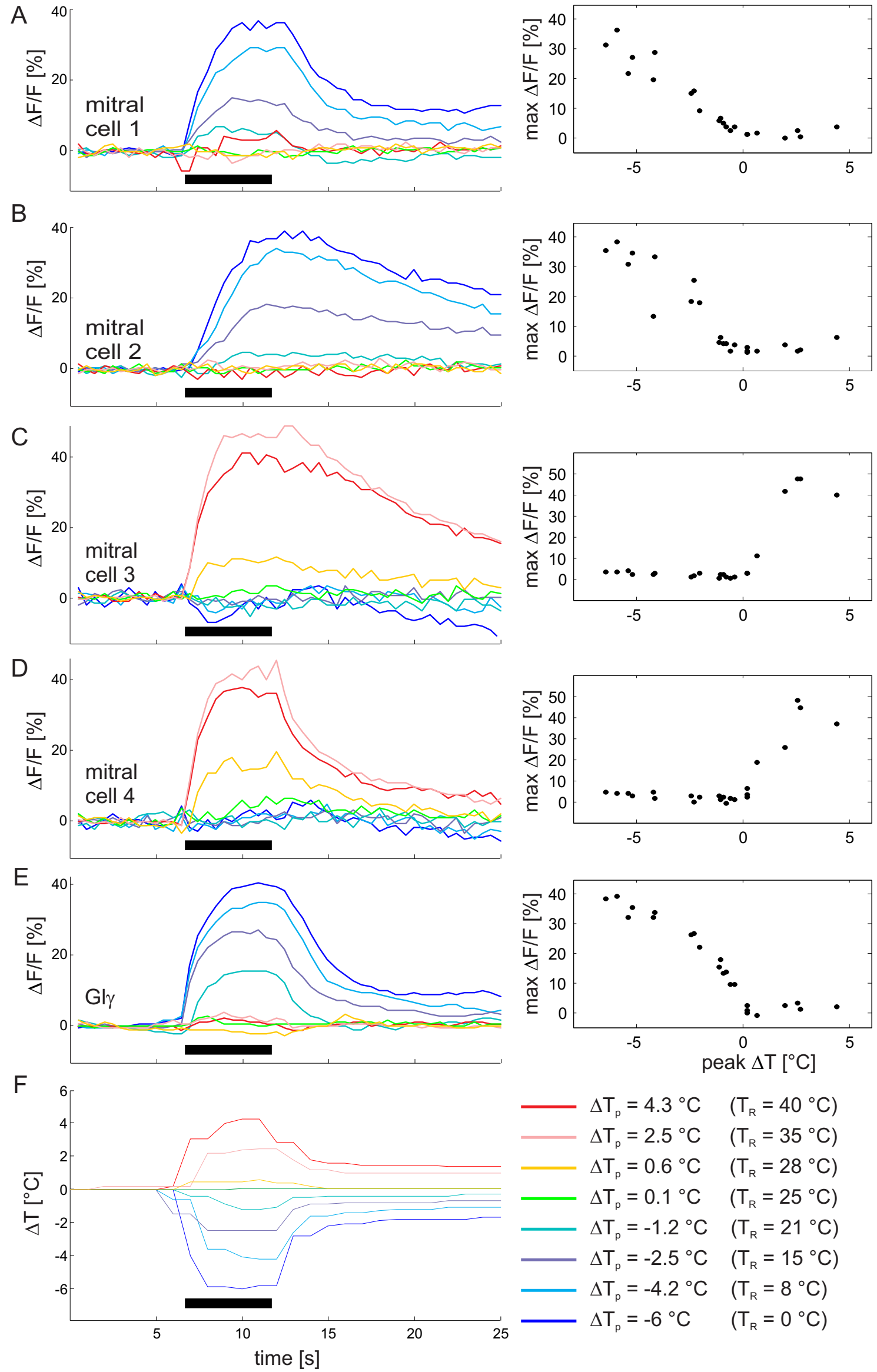

Figure 3.19: Response traces to graduated temperature stimulation within the bulk stained olfactory bulb 


\subsubsection{Responses to contralateral temperature stimulation within the olfactory bulb}

The response to temperature stimuli applied to the contralateral OE was measured after acquiring temperature responses upon ipsilateral stimulation. The ipsilateral olfactory nerve was transected and the outlet of the stimulus application system was moved from the ipsilateral to the contralateral $\mathrm{OE}$. Two of five preparations were damaged during this additional preparation procedure and were therefore excluded from the results. In two of the three remaining preparations a reaction to contralateral stimulation could be elicited. The stimulation protocol was restricted to repeated cold stimulus and applications of Ringer solution at room temperature as a negative control. The traced deviating OSN axons served again as an orientation to find Gly (Figure 3.20 B). The temperature-sensitive neuropil intermingled with deviating OSN axons in the location of Gl $\gamma$ (Figure $3.20 \mathrm{D}$ ). Both the postsynaptic glomerular meshwork and the somata of mitral cells responded to stimulation with cold Ringer solution (Figure 3.20 E). The graduated temperature stimulations were not repeated in a sufficient number to allow a conclusion about a graduated response as verified for the ipsilateral stimulation. Nevertheless these experiments clearly show that the temperature stimulation of the contralateral $\mathrm{OE}$ is relayed through the first olfactory synapse and leads to an activation of mitral cells.

\section{Figure 3.20: Responses to contralateral temperature stimulation within the olfactory bulb}

A: Basal fluo-4 signal prior to stimulation. ROI for response trace of Gly is marked with $\gamma$ and ROI for responding mitral cell soma is marked with ' $m$ '.

B: Alexa Fluor $56810 \mathrm{kDa}$ dextran signal from deviating OSN axons in red channel.

C: Spatial distribution of the response as $\Delta \mathrm{F} / \mathrm{F}$ at peak response map (colormap only in blue and green channels).

D: Overlay of responding regions and deviating OSN axons.

E: Response $\Delta \mathrm{F} / \mathrm{F}$ time traces of $\mathrm{Gl} \gamma$ and temperature sensitive mitral cell to stimulation with $\quad \Delta \mathrm{T}_{\mathrm{p}}=-2.8 \pm 0.4{ }^{\circ} \mathrm{C} \quad\left(\mathrm{T}_{\mathrm{R}}=0{ }^{\circ} \mathrm{C}\right), \quad \Delta \mathrm{T}_{\mathrm{p}}=-1.8^{\circ} \mathrm{C} \quad\left(\mathrm{T}_{\mathrm{R}}=8{ }^{\circ} \mathrm{C}\right), \quad \Delta \mathrm{T}_{\mathrm{p}}=-0.9{ }^{\circ} \mathrm{C}$ $\left(\mathrm{T}_{\mathrm{R}}=15^{\circ} \mathrm{C}\right)$ and $\Delta \mathrm{T}_{\mathrm{p}}=0.1{ }^{\circ} \mathrm{C}\left(\mathrm{T}_{\mathrm{R}}=25^{\circ} \mathrm{C}\right)$. The time intervals of the temperature stimulus are visualised with a black line below the traces. The corresponding temperature changes at the $\mathrm{OE}$ are presented as $\Delta \mathrm{T}$ time traces.

Abbreviations: $\gamma$, Gl $\gamma$; m, mitral cell; C, caudal; L, lateral; M, medial; R, rostral. 

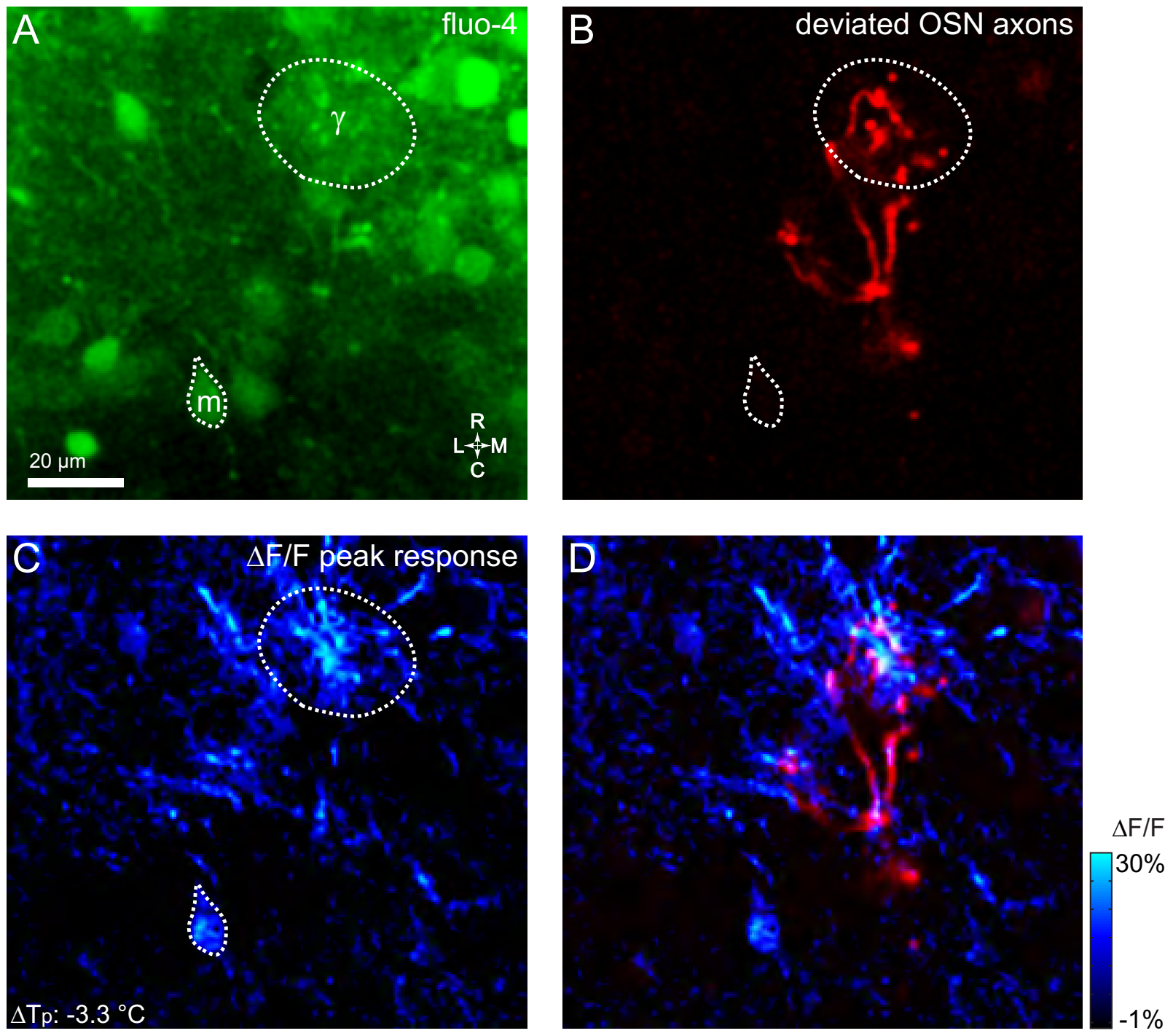

$E$
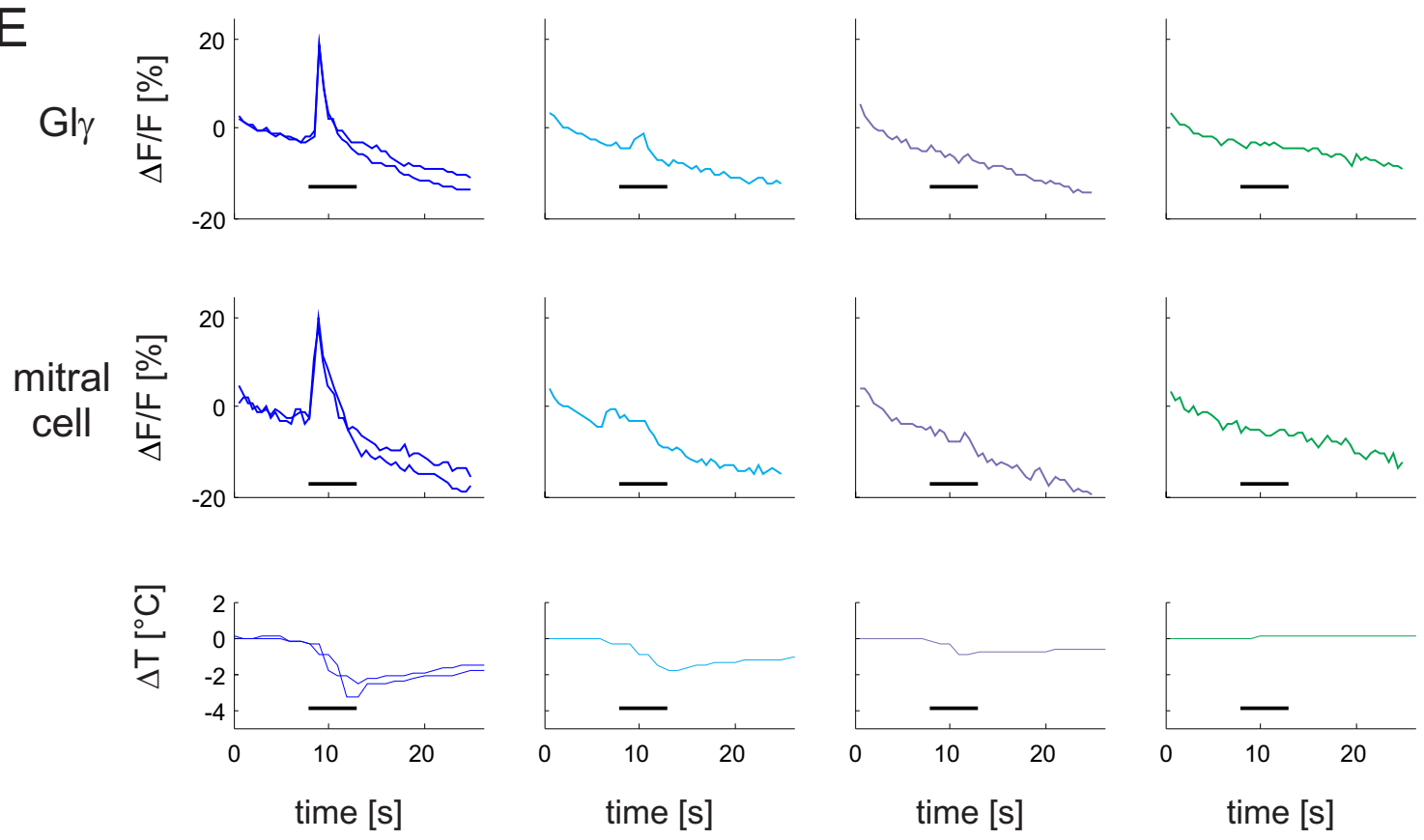

Figure 3.20: Responses to contralateral temperature stimulation within the olfactory bulb 


\subsubsection{Summary: results of calcium imaging of mitral cells}

The evaluation of dye distribution after bulk staining of the OB with AM coupled dye verified that the bolus loading of fluo-4/AM can be used to acquire transient $\left[\mathrm{Ca}^{2+}\right]_{\mathrm{i}}$ responses exclusively from the postsynaptic glomerular neuropil. The subsequent calcium imaging experiments revealed a graduated response in mitral cell somata upon stimulation of the ipsilateral OE either with cold or warm Ringer solutions. The postsynaptic terminals of Gl $\gamma$ unambiguously responded to ipsilateral stimulation with cold Ringer solution. A response of Gl $\gamma$ and mitral cell somata upon stimulation of the contralateral OE with cold Ringer solution could be demonstrated as well. 


\section{Discussion}

The results of this thesis present a novel pattern of glomerular innervation in the vertebrate olfactory system, consisting of primary olfactory fibers projecting to the contralateral $\mathrm{OB}$ via the anterior commissure and forming glomerular tufts within a defined olfactory glomerulus: Gl $\gamma$. This olfactory glomerulus did not respond to any of the tested odorants, but specifically responded to the stimulation of the OE with cold Ringer solution. The response of the Gl $\gamma$ innervating OSNs to temperature stimulation was transmitted through the first olfactory synapse and elicited a response of mitral cells, indicating that the response to temperature stimulation is relayed to higher brain centers. In addition, the results present measurements demonstrating the processing of bilateral stimuli at the level of olfactory glomeruli.

\subsection{Are axons of olfactory sensory neurons the exclusive constituents of electroporation-traced fibers?}

The introduction of a dye into the nasal cavity and consecutive electroporation stain exclusively cells that are in a direct contact with the principal cavity. Currently, there are only two known cell types within the OE of Xenopus laevis that are exposed to the environment: sustentacular cells and OSNs (Hansen et al., 1998). Of these only OSNs possess axons that project to the $\mathrm{OB}$.

Additionally to the OSN axons that project to the OB from the OE, the processes from neurons of the terminal nerve extend between the OB and the OE (von Bartheld, 2004). Several studies of the terminal nerve indicate that its processes lie deep within the OE without contact to the principal cavity: the terminal nerve neurons were not stained after application of tracers to the surface of the olfactory mucosa of elasmobranch (Hofmann and Meyer, 1995), chondrostean (Huesa et al., 2000), teleost (Szabo et al., 1991; von Bartheld et al., 1988), adult and larval Xenopus laevis (Hofmann and Meyer, 1991a; Pinelli et al., 2004). However, a single study reports staining of the terminal nerve neurons in the olfactory nerve of adult Xenopus laevis after application of biocytin to the principal cavity (Koza and Wirsig-Wiechmann, 2001). This contradicting result from the adult animal was not verified for the Xenopus laevis tadpoles (Pinelli et al., 2004). Thus, the overwhelming evidence indicates that the processes of the terminal nerve are not part of the fibers that were detected within the OB after electroporation loading of the $\mathrm{OE}$ in this thesis. 
Additionally to this morphological evidence, the responses within the traced fibers upon odorant stimulation indicate their OSN origin. A previous study of OSNs revealed that from the population of OSNs sensitive to amino acids and/or forskolin, most OSNs responded either to amino acids (47.7\%) or forskolin $(47.2 \%)$ and only $5.12 \%$ of OSNs responded to both stimuli (Manzini and Schild, 2003b). This distribution corresponds to the observed distribution of responses of traced fibers to stimulation with amino acids and forskolin in this thesis. Most of the traced fibers responded either to amino acids or forskolin; only a small region identified as G1 $\beta$ responded to both stimuli (Figure 3.10). The spatial distribution of responses to amino acids within the lateral cluster (Figure $3.10 \mathrm{C}$ ) and to forskolin within the medial cluster (Figure $3.10 \mathrm{D}$ ) corresponds to the spatial distribution of the responses that were measured within bulk stained OB slices in a previous study (Manzini et al., 2007b). This consistency within the response profiles indicates that the electroporation-traced fibers that innervate G1 $\beta$, medial and lateral clusters consist of OSN axons.

While the odorant response profiles indicate that G1 $\beta$ innervating fibers consist of OSN axons, no measurements on temperature sensitive OSNs in the $\mathrm{OE}$ are available. However, the shape of the transient $\left[\mathrm{Ca}^{2+}\right]_{i}$ response within $\mathrm{Gl} \gamma$ upon temperature stimulation was similar to the transient $\left[\mathrm{Ca}^{2+}\right]_{i}$ response within $\mathrm{Gl} \beta$ upon amino acid stimulation (Figure $3.8 \mathrm{G}$ and $\mathrm{H}$ ). This similarity of responses is an evidence for OSN axons as constituents of $\mathrm{Gl} \gamma$.

The morphological evidence and the existence of calcium responses during stimulation were sufficient to designate all fibers within the OB that were stained by electroporation loading in the OE as OSN axons in this thesis. An additional verification of fibers innervating Gl $\gamma$ as OSN axons could further support this evidence. Such verification is possible by two different approaches: either by showing temperature sensitive OSNs within the OE or by backtracing OSNs innervating Gly to the OE. A specific backtracing of Gl $\gamma$ innervating OSNs is possible by tracing of deviating fibers, as discussed in the next section. 


\subsection{Deviating axons of olfactory sensory neurons - a new olfactory pathway?}

The deviating fibers follow the extrabulbar olfactory pathway to the contralateral telencephalon via the anterior commissure and then turn rostrally to innervate the contralateral OB. To my knowledge, they represent a novel pathway in the vertebrate olfactory system. The results presented in this study unambiguously show the existence of this pathway in the olfactory system of Xenopus laevis tadpoles. Since the deviating fibers run alongside with EBOF, they were presumably detected by earlier tracings but not identified as a separate entity. The EBOF pathway itself was overlooked by neurobiologists for a long time and was first differentiated from the terminal nerve system in teleost fish by Szabo et al. (1991) and in Xenopus laevis by Hofmann and Meyer (Hofmann and Meyer, 1992). The question of whether the EBOF are a part of the terminal nerve is still debated controversially (von Bartheld, 2004).

The pathway of the EBOF in Xenopus laevis tadpoles was studied in detail by Pinelli et al. (2004). They identified the area where EBOF cross to the contralateral side as the 'anterior commissure'. The nomenclature of Pinelli et al. (2004) was adopted in this thesis. The diencephalic structure which is innervated by the EBOF and which lies on the midline just caudally from the anterior commissure at stages 47 and 52 (marked with an asterisk in Figure 3.1 and Figure 3.4) was not mentioned by Pinelli et al. (2004). However an identification of this structure was not subject of the present study.

Pinelli et al. (2004) also applied a retrograde tracer to the anterior commissure of Xenopus laevis tadoples (stages $45-66$ ) and followed the stained processes back to the OE. Since EBOF from both sides of the animals pass through the anterior commissure, both sides were traced equally. Pinelli et al. (2004) reported that no fibers terminating in tufts were found within the OB. This contradicts with the results of the current study, since the deviating fibers also run through the anterior commissure. The missing evidence of innervation of $\mathrm{Gl} \gamma$ by deviating fibers can be explained by the remarkable specificity of the contralateral innervation by deviating fibers to only a single glomerulus out of about 350 within the main OB (Manzini et al., 2007b). Most probably the innervation of Gly has been overlooked by Pinelli et al. (2004).

Pinelli et al. (2004) identified EBOF as OSN axons. The deviating fibers run alongside EBOF via the anterior commissure and were probably traced together with EBOF as 
described above. It is possible that some of the backtraced fibers that were identified as OSN axons did not belong to the EBOF but to the deviating fibers. This retrograde tracing study did not indicate sources within the OE for fibers that pass through the anterior commissure other than OSNs (Pinelli et al., 2004).

The evidence from this thesis and the retrograde tracing study by Pinelli et al. (2004) indicate that the deviating fibers are OSN axons. Additional tracing experiments to verify the OSNs as a source for deviating fibers and to explore the distribution of these OSNs within OE are possible. A specific retrograde tracing of the deviating fibers can be achieved by introducing a tracer directly within Gl $\gamma$ or along the EBOF pathway prior to the anterior commissure. In this way, the deviated fibers would be retrogradely traced towards their source within the contralateral OE. An additional verification of OSNs as a source of deviating fibers would simultaneously identify the OSNs as a source of temperature sensitive fibers, since the deviating fibers were also shown to be temperature sensitive. However, this has to be performed in future studies.

\subsection{Is Gly an exclusive target for deviating axons of olfactory sensory neurons?}

The innervation pattern of deviating OSN axons was analysed in detail in tadpoles at stage 52. Deviating OSN axons projected in six out of 16 OBs $(37.7 \%)$ into other regions beside Gl $\gamma$. The distribution of these deviating OSN axons outside Gl $\gamma$ appeared to be random and the occurrence of these fibers decreased with distance to Gl $\gamma$. Glomerular tufts formed by deviating OSN axons outside Gl $\gamma$ were restricted to the intermediate cluster (Table 1). This distribution of deviating OSN axons indicates Gl $\gamma$ as their presumable target.

At stage 47 only two OBs were imaged with high resolution which allowed a detailed investigation of the innervation pattern of deviating OSN axons. Although the deviating OSN axons innervated exclusively Gly in both cases, more specimens should be analysed to verify whether Gl $\gamma$ is the exclusive target for deviating OSN axons at stage 47. 


\subsection{Is temperature an adequate quality for the vertebrate olfactory system?}

The results in this thesis show unambiguously that OSN axon terminals and mitral cell neuropil within Gly respond upon stimulation of the ipsilateral and contralateral OE with low temperature. Additionally to the response of the glomerular neuropil, a corresponding response in somata of mitral cells was detected. The transient $\left[\mathrm{Ca}^{2+}\right]_{\mathrm{i}}$ response upon temperature stimulation was restricted to Gl $\gamma$, while the adjacent Gl $\beta$ did not respond to temperature stimulation. The shape of the transient $\left[\mathrm{Ca}^{2+}\right]_{\mathrm{i}}$ responses within Gl $\gamma$ to temperature stimulation and within G1 $\beta$ to odorant stimulation with amino acids was similar and consistent with the duration of the stimulus application.

These measurements in Xenopus laevis tadpoles are not the first indication that temperature is an adequate quality for the vertebrate olfactory system. A recent study in mouse showed that cool ambient temperatures induce strong c-Fos expression in some OSNs of the Grüneberg ganglion (Mamasuew et al., 2008). The Grüneberg ganglion is a separate component of the mammalian olfactory system and its neurons project to the so-called necklace glomeruli within the OB of the mouse (Fleischer et al., 2006; Fuss et al., 2005; Grüneberg, 1973; Koos and Fraser, 2005; Roppolo et al., 2006; Storan and Key, 2006). The necklace glomeruli of the mouse are morphologically similar to the G1 $\beta$ and Gly glomeruli of Xenopus laevis tadpoles. In both cases the olfactory glomeruli lie separate from the main glomerular clusters on the caudal rim of the OB forming a 'beads on a string' like structure.

The measurements of the OSN axons only showed responses to cold temperature stimulation while the mitral cells responded either to cold (Figure 3.19 A and B) or to warm temperature stimulations (Figure $3.19 \mathrm{C}$ and D). The majority of mitral cells $(82 \%)$ responded to cold temperature and the responding postsynaptic glomerular neuropil clearly overlapped with the presumed location of Gl $\gamma$ (Figure 3.18 A to C). The spatial distribution of the neuropil from mitral cells responding to warm temperature was less distinct and appeared more dispersed (Figure $3.18 \mathrm{E}$ ). The spatial maps of single optical slices as presented in this thesis are not sufficient to estimate how the neuropil responding to warm temperature is associated with Gly. Additional experiments with three dimensional reconstruction of dendritic trees from mitral cells responding to warm temperature using 'activity correlation imaging' (Junek et al., 2009) could verify whether these mitral cells innervate Gl $\gamma$. 
Though the spatial distribution of responses to warm temperature remain unclear, the clear responses to cold temperature at the level of the pre- and postsynaptic terminals within olfactory glomeruli and within mitral cells (the second stage of the olfactory system) indicate that temperature stimulation is processed within the olfactory system of larval Xenopus laevis. The data from this thesis, together with the available data on thermosensation within the Grüneberg ganglion in the mouse, indicate that temperature is an adequate quality for the vertebrate olfactory system.

\subsection{What is the function of the contralateral innervation?}

To date, only morphological descriptions of contralaterally projecting OSN axons were performed (Byrd and Burd, 1991; Gaudin and Gascuel, 2005; Nezlin and Schild, 2005). The response of deviating OSN axons to cold temperature stimulation is to my knowledge the first physiological description of bilateral processing in the vertebrate olfactory glomeruli. The results obtained from measurements of mitral cells and postsynaptic Gl $\gamma$ neuropil indicate that the response of contralateral OSNs (Figure 3.12) is transmitted to postsynaptic glomerular neuropil and somata of mitral cells (the second stage of the olfactory pathway, Figure 3.20).

In previous studies, it was shown that the integration of stimulus responses from both olfactory epithelia begins at the level of mitral cells: the mitral cell axons project bilaterally through the lateral olfactory tract to lateral and dorsal pallial areas and some of them innervate the contralateral OB through the anterior commissure (Eisthen, 1997; Huesa et al., 2000). A bilateral projection was also reported for the extrabulbar olfactory pathway; however, the targets for this bilateral innervation lie caudally to the anterior commissure (Hofmann and Meyer, 1989; Pinelli et al., 2004) and its function is completely unknown (von Bartheld, 2004).

Unlike EBOF, the deviating OSN axons turn rostrally from the anterior commissure and proceed rostrally to the glomerular layer, where they intermingle with ipsilateral OSN axons in Gly (Figure 3.2 and Figure 3.5). These deviating fibers appear to be restricted to Gl $\gamma$ and the results from this study indicate that this glomerulus is temperature sensitive. This correlation indicates that the unusual projection of the deviating OSN axons is tightly linked to perception of temperature.

The role of the specific contralateral innervation by deviating OSN axons must be disentangled in further experiments in conjunction with their temperature sensitivity. 
The specific morphology of the deviating fibers could help to understand the function of this contralateral innervation. The pathway of the deviating fibers over the whole length of the telencephalon to the anterior commissure and back to the contralateral OB causes a time delay between the arrival of action potentials from the ipsilateral and contralateral OE in Gl $\gamma$. The time delay depends on the additional length of the axon and the propagation speed. The additional axon length in stage 47 tadpoles is about $1 \mathrm{~mm}$ (Figure 3.1). The propagation speed in the frog olfactory nerve was measured as $0.14 \mathrm{~m} / \mathrm{s}$ (Ottoson, 1960). Therefore a time delay in the range of $7 \mathrm{~ms}$ can be expected. Additional experiments in which both olfactory epithelia are stimulated could give an insight as to whether this time delay is used to integrate the information from both olfactory epithelia within Gl $\gamma$. The integration of sensory information from bilateral receptive fields is utilised for localisation of stimulus sources in the olfactory system of humans (Porter et al., 2005), rats (Rajan et al., 2006) as well as flies (Duistermars et al., 2009). Thus the integration of temperature stimuli from both olfactory epithelia could subserve to generate their spatial representation.

\subsection{Calcium influx over the entire length of axons of olfactory sensory neurons.}

The electroporation tracing of OSN axons with fluo-4 $10 \mathrm{kDa}$ dextran revealed that transient $\left[\mathrm{Ca}^{2+}\right]_{i}$ responses are not restricted to intraglomerular axon terminals; they could be measured along the entire length of the OSN axons (Figure 3.7). The mechanism of the extraglomerular influx of $\mathrm{Ca}^{2+}$ into OSN axons is unclear and was not addressed by experiments in this thesis. However, since the voltage-gated sodium channels presumably evolved from low voltage-activated calcium channels (Anderson and Greenberg, 2001; Hille, 1989; Spafford et al., 1999; Yu and Catterall, 2003), the voltage-gated sodium channels in OSN axons of an anamniotic vertebrate, such as Xenopus laevis, may be partly permeable to $\mathrm{Ca}^{2+}$.

The described extraglomerular influx of $\mathrm{Ca}^{2+}$ into OSN axons in larval Xenopus laevis is contradictory to earlier studies in mouse (Wachowiak et al., 2004) and zebrafish (Friedrich and Korsching, 1997) where transient $\left[\mathrm{Ca}^{2+}\right]_{\mathrm{i}}$ responses were only observed in OSN axon terminals within the olfactory glomeruli. While the different observations obtained in mouse might be explained by the lower specificity of voltage-gated sodium channels in anamniotes compared to mammals, the discrepancy to the study using zebrafish might be due to different experimental conditions. 
The transient $\left[\mathrm{Ca}^{2+}\right]_{i}$ responses within zebrafish OSN axons were recorded from the OB surface using a widefield-epifluorescence microscope equipped with a CCD camera (Friedrich and Korsching, 1997). It is possible that these experimental conditions were not sensitive enough to detect and identify transient $\left[\mathrm{Ca}^{2+}\right]_{i}$ responses originating from OSN axons outside glomeruli in zebrafish. In the present study, a confocal LSM was used for signal acquisition with high spatial resolution. Thus it was possible to detect extraglomerular calcium influx within OSN axons of larval Xenopus laevis. A confocal LSM could be used for comparative experiments in zebrafish to verify whether the extraglomerular calcium influx within OSN axons can be seen in other anamniotic vertebrates.

\subsection{Selective postsynaptic staining by AM dyes}

The tracing of OSN axons combined with bulk staining of OB demonstrated that both stainings were complementary. Both bolus loading and bath incubation of Fura Red/AM and fluo-4/AM resulted in a specific staining of OB neurons. The bath incubation of fluo-4/AM was used in a previous study to explore the response profiles of olfactory glomeruli in larval Xenopus laevis (Manzini et al., 2007a). The imaged responses of the neuropil meshwork within the glomerular layer appeared to consist of both OSN axon terminals and mitral cell dendrites since application of non-NMDA and NMDA glutamate receptor antagonists 6-cyano-7-nitroquinoxaline-2,3-dione (CNQX) and D-2amino-5-phosphonovaleric acid (D-APV) in order to block the synaptic transmission between OSNs and mitral cells (Berkowicz et al., 1994; Ennis et al., 1996; Shipley and Ennis, 1996) did not suppress $\left[\mathrm{Ca}^{2+}\right]_{\mathrm{i}}$ transients completely (Manzini et al., 2007a).

The results of the present thesis indicate that the block of synaptic transmission in the study by Manzini et al. (2007a) was probably not complete and that the measured residual transient $\left[\mathrm{Ca}^{2+}\right]_{\mathrm{i}}$ responses originated from mitral cell neuropil. The response of postsynaptic glomerular neuropil at the slice surface is not exclusively caused by synaptic transmission within these superficial glomeruli. The mitral cells of larval Xenopus laevis are known to innervate more than one glomerulus (Nezlin and Schild, 2000; Nezlin and Schild, 2005; Nezlin et al., 2003). A successful synaptic transmission within a single glomerulus that is innervated by a mitral cell is sufficient to elicit a backpropagation of action potentials to the complete dendritic tree of this mitral cell, including the glomerular tufts in all glomeruli that are innervated by this mitral cell 
(Bischofberger and Jonas, 1997; Charpak et al., 2001; Junek et al., 2009). This means that the residual activity upon odorant stimulation measured after application of D-APV and CNQX to the bath solution could be caused by synaptic transmission in olfactory glomeruli that were located deep in the slice and thus not affected by application of the blocker. This residual activity was back-propagated to the imaged olfactory glomeruli at the slice surface.

A replication of the previous staining conditions supplemented by tracing of OSN axons with Alexa Fluor $56810 \mathrm{kDa}$ dextran showed no transient $\left[\mathrm{Ca}^{2+}\right]_{\mathrm{i}}$ responses within these axons (Figure 3.16). These results indicate that OSN axons are not stained by application of AM coupled fluo-4 dye and do not contribute to the transient $\left[\mathrm{Ca}^{2+}\right]_{\mathrm{i}}$ responses within the olfactory glomerular meshwork.

The transient $\left[\mathrm{Ca}^{2+}\right]_{\mathrm{i}}$ responses acquired from fluo-4 $10 \mathrm{kDa}$ dextran electroporated OSN axons demonstrate that electroporation of OSN axons did not interfere with $\left[\mathrm{Ca}^{2+}\right]_{\mathrm{i}}$ transients as response to stimulation (Figure 3.8). This indicates that the missing calcium signal is not prohibited by electroporation of the dextran coupled dye. The missing calcium signal is apparently caused by the absence of fluo-4 within OSN axons after bulk staining with fluo-4/AM.

To ensure that OSN axons were not stained by fluo-4/AM, the bulk staining was repeated with a different calcium sensitive dye. Neither a bath incubation nor bolus loading of Fura Red/AM resulted in any fluorescence signal within OSN axons (Figure 3.14 and Figure 3.13). These results demonstrate that bulk staining of OB with AM coupled dyes is restricted to postsynaptic glomerular neuropil within olfactory glomeruli.

One likely explanation for the different staining levels in the OSN axons and OB neurons is the extremely different ratio of volume to surface exposed to the dye solution. While the OB neurons are exposed with their somata and dendritic processes to the dye solution, only the terminal part of OSN axons is exposed to the dye solution and the de-esterificated dye is further diluted by diffusion into the olfactory nerve. Further, the uptake of AM coupled dyes is known to be specific for different neuron types (Kirischuk and Verkhratsky, 1996). In particular, the AM coupled dyes are subject to extrusion by multidrug resistance P-glycoprotein and multidrug-resistance-associated proteins in the OSNs of larval Xenopus laevis (Manzini and Schild, 2003a). In the 
present study, the multidrug-resistance-associated proteins were blocked by MK571. However, it is possible that unblocked transporters, such as multidrug resistance P-glycoprotein, specifically impair the staining of OSN axons with AM coupled dyes.

The specific staining of OB neuron dendrites after bulk staining with AM coupled dyes offers the possibility to simultaneously measure transient $\left[\mathrm{Ca}^{2+}\right]_{\mathrm{i}}$ responses of OSN axon terminals and OB neuron dendrites with two different calcium sensitive dyes. Some preliminary experiments with Xenopus laevis tadpoles that were electroporated with fluo-4 $10 \mathrm{kDa}$ dextran into the $\mathrm{OE}$ and bolus loaded with Rhod-2/AM in the OB were performed. It was possible to simultaneously measure $\left[\mathrm{Ca}^{2+}\right]_{\mathrm{i}}$ responses of OSN axon terminals and OB neuron dendrites in G1 $\beta$ to odorant stimulation (data not shown). This double staining technique will be a powerful tool to unravel the functioning of olfactory glomeruli.

\subsection{Perspective}

This study revealed thermoreception within the olfactory system of an anamniotic vertebrate. The processing of the temperature stimuli is performed bilaterally at the level of Gl $\gamma$. This olfactory glomerulus is innervated by temperature sensitive OSN axons emerging from the ipsilateral as well as the contralateral OE.

This thesis gives a first insight into the perception of temperature by the olfactory system and opens a wide array of intriguing questions. Future studies need to address

the investigation of temperature sensitive OSNs in the OE, the mechanisms of cold or warm temperature reception, the adaptation to different environment temperatures and the role of the bilateral innervation of Gl $\gamma$. 


\section{List of Abbreviations}

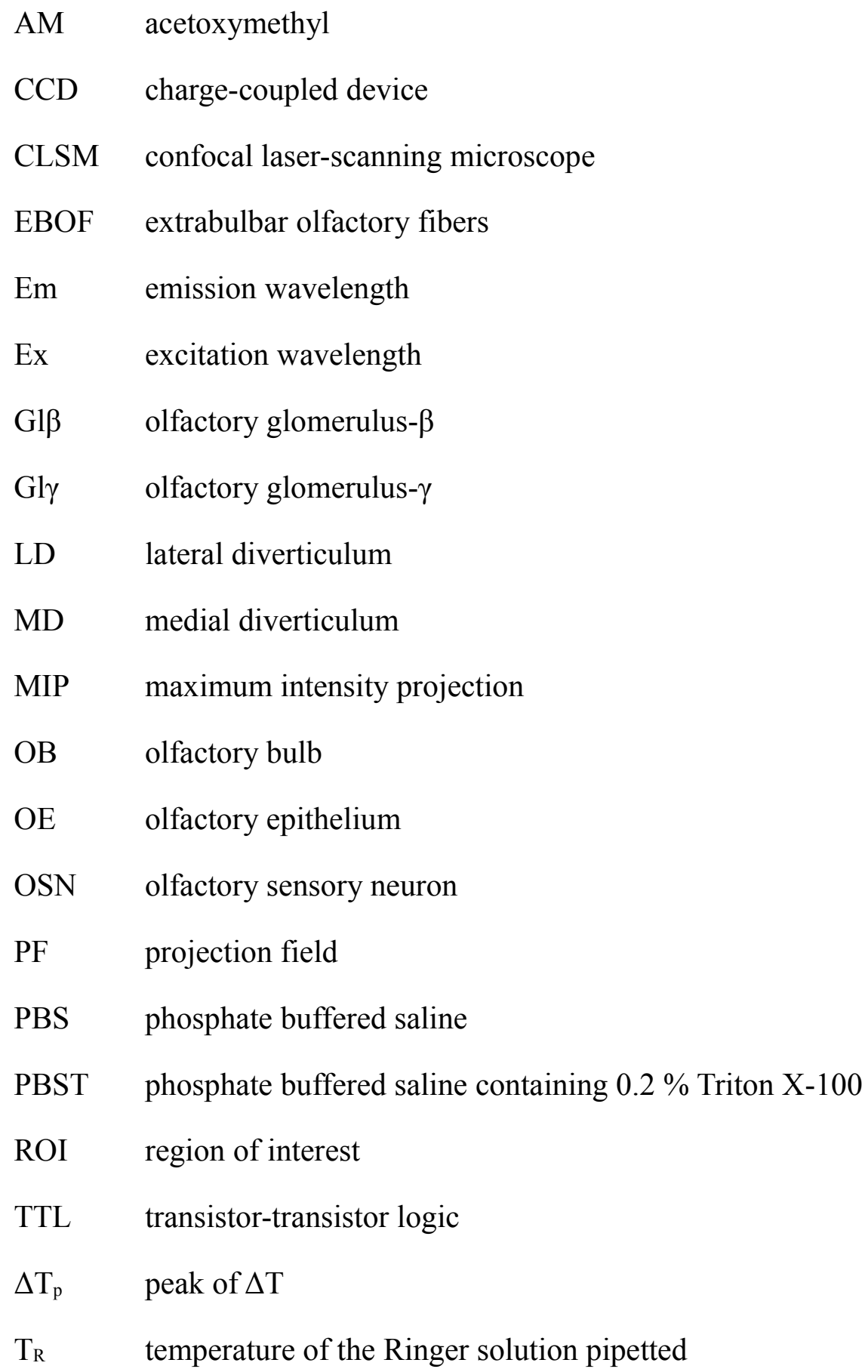

Abbreviations exclusively used in figures are explained in the respective figure caption. 


\section{References}

Ache, B. W. and Young, J.M. (2005). Olfaction: diverse species, conserved principles. Neuron, 48, 417-30.

Altner, H. (1962). Untersuchungen über Leistungen und Bau der Nase des südafrikanischen Krallenfrosches Xenopus laevis (Daudin, 1803). J. Comp. Physiol. A Neuroethol. Sens. Neural. Behav. Physiol., 45, 272-306.

Anderson, P. A. and Greenberg, R. M. (2001). Phylogeny of ion channels: clues to structure and function. Comp Biochem Physiol B Biochem Mol Biol, 129, 17-28.

Baxi, K. N., Dorries, K. M. and Eisthen, H. L. (2006). Is the vomeronasal system really specialized for detecting pheromones? Trends Neurosci, 29, 1-7.

Berkowicz, D. A., Trombley, P. Q. and Shepherd, G. M. (1994). Evidence for glutamate as the olfactory receptor cell neurotransmitter. $J$ Neurophysiol, 71, 2557-61.

Bestman, J. E., Ewald, R. C., Chiu, S. and Cline, H. T. (2006). In vivo single-cell electroporation for transfer of DNA and macromolecules. Nat Protoc, 1, 1267-72.

Bischofberger, J. and Jonas, P. (1997). Action potential propagation into the presynaptic dendrites of rat mitral cells. $J$ Physiol, 504 (Pt 2), 359-65.

Bonnot, A., Mentis, G. Z., Skoch, J. and O'Donovan, M. J. (2005). Electroporation loading of calcium-sensitive dyes into the CNS. J Neurophysiol, 93, 1793-808.

Boutin, C., Diestel, S., Desoeuvre, A., Tiveron, M. and Cremer, H. (2008). Efficient in vivo electroporation of the postnatal rodent forebrain. PLoS One, 3, e1883.

Breer, H. and Strotmann, J. (2005). The septal organ: a 'mini-nose' with dual function?. ChemoSense, 7, 2-7.

Burd, G. D. (1991). Development of the olfactory nerve in the African clawed frog, Xenopus laevis: I. Normal development. J Comp Neurol, 304, 123-34.

Byrd, C. A. and Burd, G. D. (1991). Development of the olfactory bulb in the clawed frog, Xenopus laevis: a morphological and quantitative analysis. J Comp Neurol, 314, 79-90.

Caprio, J. and Byrd, R. P. J. (1984). Electrophysiological evidence for acidic, basic, and neutral amino acid olfactory receptor sites in the catfish. $J$ Gen Physiol, 84, 403-22.

Charpak, S., Mertz, J., Beaurepaire, E., Moreaux, L. and Delaney, K. (2001). Odorevoked calcium signals in dendrites of rat mitral cells. Proc Natl Acad Sci U S A, 98, $1230-4$.

Czesnik, D., Kuduz, J., Schild, D. and Manzini, I. (2006). ATP activates both receptor and sustentacular supporting cells in the olfactory epithelium of Xenopus laevis tadpoles. Eur J Neurosci, 23, 119-28. 
Czesnik, D., Schild, D., Kuduz, J. and Manzini, I. (2007). Cannabinoid action in the olfactory epithelium. Proc Natl Acad Sci U S A, 104, 2967-72.

Duistermars, B. J., Chow, D. M. and Frye, M. A. (2009). Flies require bilateral sensory input to track odor gradients in flight. Curr Biol, 19, 1301-7.

Ebbesson, S. O., Bazer, G. T. \& Jane, J.A. (1986). Some primary olfactory axons project to the contralateral olfactory bulb in Xenopus laevis. Neurosci Lett, 65, 234-8.

Eisthen, H. L. (1997). Evolution of vertebrate olfactory systems. Brain Behav Evol, 50, 222-33.

Eisthen, H. L. (2002). Why are olfactory systems of different animals so similar? Brain Behav Evol, 59, 273-93.

Eisthen, H. L. and Wyatt, T.D. (2006). The vomeronasal system and pheromones. Curr Biol, 16, R73-4.

Ennis, M., Zimmer, L. A. and Shipley, M. T. (1996). Olfactory nerve stimulation activates rat mitral cells via NMDA and non-NMDA receptors in vitro. Neuroreport, 7, 989-92.

Falk, J., Drinjakovic, J., Leung, K. M., Dwivedy, A., Regan, A. G., Piper, M. and Holt, C.E. (2007). Electroporation of cDNA/Morpholinos to targeted areas of embryonic CNS in Xenopus. BMC Dev Biol, 7, 107.

Fleischer, J., Hass, N., Schwarzenbacher, K., Besser, S. and Breer, H. (2006). A novel population of neuronal cells expressing the olfactory marker protein (OMP) in the anterior/dorsal region of the nasal cavity. Histochem Cell Biol, 125, 337-49.

Freitag, J., Krieger, J., Strotmann, J. and Breer, H. (1995). Two classes of olfactory receptors in Xenopus laevis. Neuron, 15, 1383-92.

Freitag, J., Ludwig, G., Andreini, I., Rössler, P. and Breer, H. (1998). Olfactory receptors in aquatic and terrestrial vertebrates. J Comp Physiol A, 183, 635-50.

Friedrich, R. W. and Korsching, S. I. (1997). Combinatorial and chemotopic odorant coding in the zebrafish olfactory bulb visualized by optical imaging. Neuron, 18, 737-52.

Friedrich, R. W. and Korsching, S. I. (1998). Chemotopic, combinatorial, and noncombinatorial odorant representations in the olfactory bulb revealed using a voltagesensitive axon tracer. $J$ Neurosci, 18, 9977-88.

Fuss, S. H., Omura, M. and Mombaerts, P. (2005). The Grueneberg ganglion of the mouse projects axons to glomeruli in the olfactory bulb. Eur J Neurosci, 22, 2649-54.

Gasser, H. S. (1956). Olfactory nerve fibers. J Gen Physiol, 39, 473-96.

Gaudin, A. and Gascuel, J. (2005). 3D atlas describing the ontogenic evolution of the primary olfactory projections in the olfactory bulb of Xenopus laevis. J Comp Neurol, 489, 403-24. 
Gee, K. R., Brown, K. A., Chen, W. N., Bishop-Stewart, J., Gray, D. and Johnson, I. (2000). Chemical and physiological characterization of fluo-4 $\mathrm{Ca}(2+)$-indicator dyes. Cell Calcium, 27, 97-106.

Gekeler, V., Ise, W., Sanders, K. H., Ulrich, W. R. and Beck, J. (1995). The leukotriene LTD4 receptor antagonist MK571 specifically modulates MRP associated multidrug resistance. Biochem Biophys Res Commun, 208, 345-52.

Gliem, S., Schild, D. and Manzini, I. (2009). Highly specific responses to amine odorants of individual olfactory receptor neurons in situ. Eur J Neurosci, 29, 2315-26.

Graziadei, P. P. (1973). Cell dynamics in the olfactory mucosa. Tissue Cell, 5, 113-31.

Griff, E. R., Greer, C. A., Margolis, F., Ennis, M. and Shipley, M. T. (2000). Ultrastructural characteristics and conduction velocity of olfactory receptor neuron axons in the olfactory marker protein-null mouse. Brain Res, 866, 227-36.

Gonzalez, F. \& Perez, R. (1998). Neural mechanisms underlying stereoscopic vision. Prog Neurobiol, 55, 191-224.

Grosmaitre, X., Santarelli, L. C., Tan, J., Luo, M. and Ma, M. (2007). Dual functions of mammalian olfactory sensory neurons as odor detectors and mechanical sensors. Nat Neurosci, 10, 348-54.

Grüneberg, H. (1973). A ganglion probably belonging to the N. terminalis system in the nasal mucosa of the mouse. Z Anat Entwicklungsgesch, 140, 39-52.

Haas, K., Jensen, K., Sin, W. C., Foa, L. and Cline, H. T. (2002). Targeted electroporation in Xenopus tadpoles in vivo - from single cells to the entire brain. Differentiation, 70, 148-54.

Hansen, A., Reiss, J. O., Gentry, C. L. and Burd, G. D. (1998). Ultrastructure of the olfactory organ in the clawed frog, Xenopus laevis, during larval development and metamorphosis. J Comp Neurol, 398, 273-88.

Hassenklöver, T., Kurtanska, S., Bartoszek, I., Junek, S., Schild, D. and Manzini, I. (2008). Nucleotide-induced $\mathrm{Ca} 2+$ signaling in sustentacular supporting cells of the olfactory epithelium. Glia, 56, 1614-24.

Heitzmann, H. \& Richards, F.M. (1974). Use of the avidin-biotin complex for specific staining of biological membranes in electron microscopy. Proc Natl Acad Sci U S A, 71, 3537-41.

Hille, B. (1989). The Sharpey-Schafer Lecture. Ionic channels: evolutionary origins and modern roles. QJ Exp Physiol, 74, 785-804.

Hofmann, M. H. and Meyer, D. L. (1989). Central projections of the nervus terminalis in four species of amphibians. Brain Behav Evol, 34, 301-7.

Hofmann, M. H. and Meyer, D. L. (1991a). Subdivisions of the terminal nerve in Xenopus laevis. J Exp Zool, 259, 324-9. 
Hofmann, M. H. and Meyer, D. L. (1991b). Functional subdivisions of the olfactory system correlate with lectin-binding properties in Xenopus. Brain Res, 564, 344-7.

Hofmann, M. H. and Meyer, D. L. (1992). Peripheral origin of olfactory nerve fibers bypassing the olfactory bulb in Xenopus laevis. Brain Res, 589, 161-3.

Hofmann, M. H. and Meyer, D. L. (1995). The extrabulbar olfactory pathway: primary olfactory fibers bypassing the olfactory bulb in bony fishes? Brain Behav Evol, 46, 37888 .

Huesa, G., Anadón, R. and Yáñez, J. (2000). Olfactory projections in a chondrostean fish, Acipenser baeri: an experimental study. J Comp Neurol, 428, 145-58.

Jakob, I., Hauser, I. A., Thévenod, F. and Lindemann, B. (1998). MDR1 in taste buds of rat vallate papilla: functional, immunohistochemical, and biochemical evidence. $\mathrm{Am} J$ Physiol, 274, C182-91.

Junek, S., Chen, T., Alevra, M. and Schild, D. (2009). Activity correlation imaging: visualizing function and structure of neuronal populations. Biophys J, 96, 3801-9.

Kang, J. and Caprio, J. (1995). In vivo responses of single olfactory receptor neurons in the channel catfish, Ictalurus punctatus. J Neurophysiol, 73, 172-7.

King, A. J., Schnupp, J. W. \& Doubell, T. P. (2001). The shape of ears to come: dynamic coding of auditory space. Trends Cogn Sci, 5, 261-270.

Kirischuk, S. and Verkhratsky, A. (1996). [Ca2+]i recordings from neural cells in acutely isolated cerebellar slices employing differential loading of the membranepermeant form of the calcium indicator fura-2. Pflugers Arch, 431, 977-83.

Kiseleva, E. I. (1995). [Natural amino acids as effective stimuli evoking chemoreceptordirected behavior in anuran tadpoles]. Zh Obshch Biol, 56, 329-45.

Konishi, M. (2000). Study of sound localization by owls and its relevance to humans. Comp Biochem Physiol A Mol Integr Physiol, 126, 459-69.

Koos, D. S. and Fraser, S. E. (2005). The Grueneberg ganglion projects to the olfactory bulb. Neuroreport, 16, 1929-32.

Koza, J. M. and Wirsig-Wiechmann, C. R. (2001). A subpopulation of nervus terminalis neurons projects to the olfactory mucosa in Xenopus laevis. J Neurosci Res, 66, 8-15.

Kreitzer, A. C., Gee, K. R., Archer, E. A. and Regehr, W. G. (2000). Monitoring presynaptic calcium dynamics in projection fibers by in vivo loading of a novel calcium indicator. Neuron, 27, 25-32.

Mamasuew, K., Breer, H. and Fleischer, J. (2008). Grueneberg ganglion neurons respond to cool ambient temperatures. Eur J Neurosci, 28, 1775-85.

Manzini, I. and Schild, D. (2003a). Multidrug resistance transporters in the olfactory receptor neurons of Xenopus laevis tadpoles. J Physiol, 546, 375-85. 
Manzini, I. and Schild, D. (2003b). cAMP-independent olfactory transduction of amino acids in Xenopus laevis tadpoles. J Physiol, 551, 115-23.

Manzini, I. and Schild, D. (2004). Classes and narrowing selectivity of olfactory receptor neurons of Xenopus laevis tadpoles. J Gen Physiol, 123, 99-107.

Manzini, I., Brase, C., Chen, T. and Schild, D. (2007a). Response profiles to amino acid odorants of olfactory glomeruli in larval Xenopus laevis. J Physiol, 581, 567-79.

Manzini, I., Heermann, S., Czesnik, D., Brase, C., Schild, D. and Rössler, W. (2007b). Presynaptic protein distribution and odour mapping in glomeruli of the olfactory bulb of Xenopus laevis tadpoles. Eur J Neurosci, 26, 925-34.

Manzini, I., Rössler, W. and Schild, D. (2002). cAMP-independent responses of olfactory neurons in Xenopus laevis tadpoles and their projection onto olfactory bulb neurons. $J$ Physiol, 545, 475-84.

Manzini, I., Schweer, T. and Schild, D. (2008). Improved fluorescent (calcium indicator) dye uptake in brain slices by blocking multidrug resistance transporters. J Neurosci Methods, 167, 140-7.

Marshall, D. A. and Maruniak, J.A. (1986). Masera's organ responds to odorants. Brain Res, 366, 329-32.

Meyer, D. L., Jadhao, A. G., Bhargava, S. and Kicliter, E. (1996). Bulbar representation of the 'water-nose' during Xenopus ontogeny. Neurosci Lett, 220, 109-12.

Mezler, M., Konzelmann, S., Freitag, J., Rössler, P. and Breer, H. (1999). Expression of olfactory receptors during development in Xenopus laevis. J Exp Biol, 202, 365-76.

Mori, K., Takahashi, Y. K., Igarashi, K. M. and Yamaguchi, M. (2006). Maps of odorant molecular features in the mammalian olfactory bulb. Physiol Rev, 86, 409-33.

Mousley, A., Polese, G., Marks, N. J. and Eisthen, H. L. (2006). Terminal nerve-derived neuropeptide y modulates physiological responses in the olfactory epithelium of hungry axolotls (Ambystoma mexicanum). J Neurosci, 26, 7707-17.

Nagayama, S., Zeng, S., Xiong, W., Fletcher, M. L., Masurkar, A. V., Davis, D. J., Pieribone, V. A. and Chen, W. R. (2007). In vivo simultaneous tracing and $\mathrm{Ca}(2+)$ imaging of local neuronal circuits. Neuron, 53, 789-803.

Nevian, T. and Helmchen, F. (2007). Calcium indicator loading of neurons using singlecell electroporation. Pflugers Arch, 454, 675-88.

Nezlin, L. P. and Schild, D. (2000). Structure of the olfactory bulb in tadpoles of Xenopus laevis. Cell Tissue Res, 302, 21-9.

Nezlin, L. P. and Schild, D. (2005). Individual olfactory sensory neurons project into more than one glomerulus in Xenopus laevis tadpole olfactory bulb. J Comp Neurol, 481, 233-9. 
Nezlin, L. P., Heermann, S., Schild, D. and Rössler, W. (2003). Organization of glomeruli in the main olfactory bulb of Xenopus laevis tadpoles. J Comp Neurol, 464, 257-68.

Nieuwkoop, P. D. and Faber, J. (1994). Normal Table of Xenopus laevis (Daudin). Garland Publishing Inc, New York.

Niimura, Y. and Nei, M. (2006). Evolutionary dynamics of olfactory and other chemosensory receptor genes in vertebrates. J Hum Genet, 51, 505-17.

Ottoson, D (1960), Comparison of slow potentials evoked in the frog's nasal mucosa and olfactory bulb by natural stimulation. Acta Physiologica Scandinavica, 47, 149-159.

Phillips, S. and Griff, E. R. (2002). Impulse conduction of olfactory receptor neuron axons. Microsc Res Tech, 58, 161-7.

Pinelli, C., D'Aniello, B., Polese, G. and Rastogi, R. K. (2004). Extrabulbar olfactory system and nervus terminalis FMRFamide immunoreactive components in Xenopus laevis ontogenesis. J Chem Neuroanat, 28, 37-46.

Porter, J., Anand, T., Johnson, B., Khan, R. M. and Sobel, N. (2005). Brain mechanisms for extracting spatial information from smell. Neuron, 47, 581-92.

Rajan, R., Clement, J. P. and Bhalla, U. S. (2006). Rats smell in stereo. Science, 311, 666-70.

Reiss, J. O. and Burd, G. D. (1997). Metamorphic remodeling of the primary olfactory projection in Xenopus: developmental independence of projections from olfactory neuron subclasses. J Neurobiol, 32, 213-22.

Rolen, S. H. and Caprio, J. (2007). Processing of bile salt odor information by single olfactory bulb neurons in the channel catfish. J Neurophysiol, 97, 4058-68.

Rolen, S. H. and Caprio, J. (2008). Bile salts are effective taste stimuli in channel catfish. J Exp Biol, 211, 2786-91.

Rolen, S. H., Sorensen, P. W., Mattson, D. and Caprio, J. (2003). Polyamines as olfactory stimuli in the goldfish Carassius auratus. J Exp Biol, 206, 1683-96.

Roppolo, D., Ribaud, V., Jungo, V. P., Lüscher, C. and Rodriguez, I. (2006). Projection of the Grüneberg ganglion to the mouse olfactory bulb. Eur J Neurosci, 23, 2887-94.

Sato, K. and Suzuki, N. (2001). Whole-cell response characteristics of ciliated and microvillous olfactory receptor neurons to amino acids, pheromone candidates and urine in rainbow trout. Chem Senses, 26, 1145-56.

Schild, D. (1985). A computer-controlled device for the application of odours to aquatic animals. J Electrophysiol Techn, 12, 71-79.

Scott, J. W. and Sherrill, L. (2008). Effects of odor stimulation on antidromic spikes in olfactory sensory neurons. J Neurophysiol, 100, 3074-85. 
Shipley, M. T. and Ennis, M. (1996). Functional organization of olfactory system. J Neurobiol, 30, 123-76.

Spafford, J. D., Spencer, A. N. and Gallin, W. J. (1999). Genomic organization of a voltage-gated $\mathrm{Na}+$ channel in a hydrozoan jellyfish: insights into the evolution of voltage-gated $\mathrm{Na}+$ channel genes. Receptors Channels, 6, 493-506.

Spehr, M. and Munger, S.D. (2009). Olfactory receptors: G protein-coupled receptors and beyond. J Neurochem, 109, 1570-83.

Storan, M. J. and Key, B. (2006). Septal organ of Grüneberg is part of the olfactory system. J Comp Neurol, 494, 834-44.

Szabo, T., Blähser, S., Denizot, J. P. and Ravaille-Véron, M. (1991). Extra-bulbar primary olfactory projection in teleost fishes. C R Acad Sci III, 312, 555-60.

Tabor, R., Yaksi, E., Weislogel, J. and Friedrich, R.W. (2004). Processing of odor mixtures in the zebrafish olfactory bulb. J Neurosci, 24, 6611-20.

Teruel, M. N. and Meyer, T. (1997). Electroporation-induced formation of individual calcium entry sites in the cell body and processes of adherent cells. Biophys $J, 73,1785-$ 96.

von Bartheld, C. S. (2004). The terminal nerve and its relation with extrabulbar "olfactory" projections: lessons from lampreys and lungfishes. Microsc Res Tech, 65, $13-24$.

von Bartheld, C. S., Claas, B., Münz, H. and Meyer, D. L. (1988). Primary olfactory projections and the nervus terminalis in the African lungfish: implications for the phylogeny of cranial nerves. Am J Anat, 182, 325-34.

Wachowiak, M., Denk, W. and Friedrich, R.W. (2004). Functional organization of sensory input to the olfactory bulb glomerulus analyzed by two-photon calcium imaging. Proc Natl Acad Sci U S A, 101, 9097-102.

Weiss, G. (1986). Die Struktur des Geruchsorgans und des Telencephalons beim südafrikanischen Krallenfrosch Xenopus laevis (Daudin) und ihre Veränderungen während der Metamorphose. Dissertation, University of Regensburg.

Yaksi, E., Judkewitz, B. and Friedrich, R.W. (2007). Topological reorganization of odor representations in the olfactory bulb. PLoS Biol, 5, e178.

Yan, Z., Tan, J., Qin, C., Lu, Y., Ding, C. \& Luo, M. (2008). Precise circuitry links bilaterally symmetric olfactory maps. Neuron, 58, 613-24.

Yu, F. H. and Catterall, W.A. (2003). Overview of the voltage-gated sodium channel family. Genome Biol, 4, 207. 


\section{Acknowledgements}

The present thesis was carried out under the supervision of Prof. Dr. Dr. Detlev Schild at the Department of Neurophysiology and Cellular Biophysics at the Institute of Physiology of the University of Göttingen, Germany.

I am grateful to:

Prof. Dr. Dr. Schild for the opportunity to work in his department and the continuous support throughout my work;

Prof. Dr. Heinrich and Prof. Dr. Müller from my thesis committee for their helpful advices and evaluation of this thesis;

Dr. Ivan Manzini and Dr. Andre Zeug for their critical discussions and important impacts on this thesis;

Dr. Stephan Junek for his MATLAB expertise and scripts;

Thorsten Nägel and Jens Kowalski from the electronic workshop for all 'custombuilt devices';

my friends and colleagues from the lab Dr. Bei-Jung Lin, Cordula Spinhoff, Dr. Dirk Czesnik, Dr. Esther Breunig, Gudrun Federkeil, Guobin Bao, Josko Kunduz, Mihai Alevra, Sebastian Gliem, Stefanie Ludwig and Dr. Tsai-Wen Chen.

A special thank to PD Dr. Catherina G. Becker, Dr. Ivan Manzini, Dr. Stephan Junek, Thomas Hassenklöver and Dr. Arwed Weigel for proof-reading this thesis and providing valuable comments and improvements. 


\section{Curriculum Vitae}

\section{Personal data}

Name

Eugen Kludt

Date of birth

03.12 .1979

Place of birth

Alma-Ata, Kazakhstan

Nationality

German

Email

ekludt@gwdg.de

\section{Education}

since 2008

Member of the doctoral program "Molecular Physiology of the Brain" at Göttingen Graduate School for Neurosciences and Molecular Biosciences

since 2005

PhD student in the laboratory of Prof. Dr. Dr. Schild,

Department of Neurophysiology and Cellular Biophysics,

University of Göttingen, Germany

$2000-2005$

Study of Biology at the University of Hamburg, Germany

Diploma work at the

Zentrum für Molekulare Neurobiologie, Hamburg

Subject: „Untersuchungen zur Bedeutung des Zellerkennungsmoleküls L1.1 für die axonale Regeneration der Retinaganglienzellen des adulten Zebrafisches (Danio rerio, Hamilton 1822)“

$1991-1999$

Gymnasium Hamm in Hamburg, Germany 